Supporting Information

\title{
Synthesis of Bistramide A
}

Alexander V. Statsuk, Dong Liu and Sergey A. Kozmin*

Department of Chemistry, The University of Chicago

5735 South Ellis Ave, Chicago, Illinois 60637

skozmin@uchicago.edu 


\section{General}

Methanol (HPLC grade), toluene (HPLC grade), ethyl acetate (ACS grade, and HPLC grade), hexane (ACS grade and HPLC grade), acetonitrile (HPLC grade), chloroform (HPLC grade) were purchased from Fisher Scientific and used without further purification. DMSO (anhydrous grade), DMF (anhydrous grade) were purchased from ACROS. Dichloromethane, diethyl ether and benzene were purified by passing over activated alumina. Tetrahydrofuran was purified by distillation from sodium benzophenone. Commercially available reagents were used without further purification. Reactions were monitored by thin layer chromatography (TLC) using Whatman precoated silica gel plates. Flash column chromatography was performed over ultra pure silica gel (230-400 mesh) from Silicycle. ${ }^{1} \mathrm{H}$ NMR and ${ }^{13} \mathrm{C}$ NMR spectra were recorded on Bruker DMX-500 spectrometers using residual solvent peaks as an internal standard. Infrared spectra (IR) were recorded with Nicolet FTIR spectrometer and are reported in reciprocal centimeter $\left(\mathrm{cm}^{-1}\right)$. Optical rotations were measured with JASCO DIP-1000 digital polarimeter, using the sodium D line. Mass Spectra were recorded with Agilent 1100 LCMS; APCI or API-ES, POS, SCAN, 70, with methanol or dichloromethane as the eluting solvent.

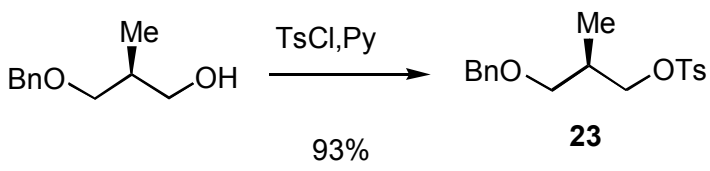

Tosylate 23. A solution of known 3-benzyloxy-2-methyl-1-propanol ${ }^{1}(11.2 \mathrm{~g}, 62.2 \mathrm{mmol}), \mathrm{TsCl}(23.7 \mathrm{~g}$, $124.4 \mathrm{mmol})$ and pyridine $(13.7 \mathrm{~mL}, 168 \mathrm{mmol})$ in $\mathrm{CH}_{2} \mathrm{Cl}_{2}(120 \mathrm{~mL})$ was stirred for $20 \mathrm{~h}$ at room temperature. A solution of pyridine $(30 \mathrm{~mL})$ in $\mathrm{CH}_{2} \mathrm{Cl}_{2}(100 \mathrm{~mL})$ was added. After $20 \mathrm{~min}$, the resulting mixture was washed with water $(100 \mathrm{~mL}), \mathrm{HCl}(1 \mathrm{M}$ solution, $100 \mathrm{~mL}), \mathrm{NaHCO}_{3}$ (saturated solution, 100 $\mathrm{mL}$ ), filtered, dried over $\mathrm{MgSO}_{4}$ and concentrated in vacuo. The crude product was purified by flash

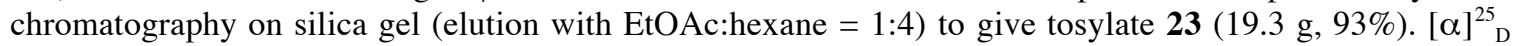
$-4.2^{\circ},\left(c\right.$ 3.0, $\left.\mathrm{CHCl}_{3}\right)$; ${ }^{1} \mathrm{H}$ NMR $\left(500 \mathrm{MHz}, \mathrm{CDCl}_{3}\right) \square 0.93(\mathrm{~d}, 3 \mathrm{H}, J=6.9 \mathrm{~Hz}), 2.05-2.13(\mathrm{~m}, 1 \mathrm{H}), 2.41$ (s, $3 \mathrm{H}), 3.31(\mathrm{dd}, 1 \mathrm{H}, J=9.0,6.5 \mathrm{~Hz}), 3.35(\mathrm{dd}, 1 \mathrm{H}, J=9.0,5.0 \mathrm{~Hz}), 3.98(\mathrm{dd}, 1 \mathrm{H}, J=9.5,5.5 \mathrm{~Hz}), 4.04(\mathrm{dd}$, $1 \mathrm{H}, J=9.5,5.5 \mathrm{~Hz}), 4.39(\mathrm{~s}, 2 \mathrm{H}), 7.21-7.35(\mathrm{~m}, 7 \mathrm{H}), 7.73-7.81(\mathrm{~m}, 2 \mathrm{H}) ;{ }^{13} \mathrm{C} \mathrm{NMR}\left(125 \mathrm{MHz}, \mathrm{CDCl}_{3}\right)$ 13.5, 21.6, 33.6, 71.0, 72.1, 73.0, 127.4, 127.5, 127.8, 128.3, 129.7, 132.9, 138.1, 144.6; IR $\left(\mathrm{CHCl}_{3}\right)$ 2855, $1356,1189,1175,1095,939,812,736,697 \mathrm{~cm}^{-1} ; \mathrm{MS}(\mathrm{CI})$ calculated for $\mathrm{C}_{18} \mathrm{H}_{22} \mathrm{O}_{4} \mathrm{~S} 334.1\left(\mathrm{M}^{+}\right)$, found $335.1(\mathrm{M}+1)$.
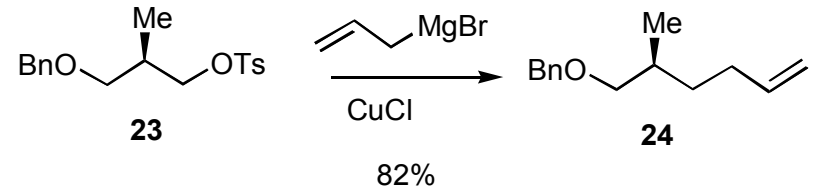

Alkene 24. To a suspension of $\mathrm{CuCl}(1.14 \mathrm{~g}, 11.5 \mathrm{mmol})$ in ether $(150 \mathrm{~mL})$ was added allyl magnesium bromide (1 M solution in ether, $116 \mathrm{~mL}, 116 \mathrm{mmol}$ ) at $0{ }^{\circ} \mathrm{C}$. After $10 \mathrm{~min}$, a solution of tosylate $23(19.3$ $\mathrm{g}, 57.7 \mathrm{mmol})$ in ether $(50 \mathrm{~mL})$ was added. The mixture was stirred for $12 \mathrm{~h}$ at room temperature, quenched with $\mathrm{NH}_{4} \mathrm{Cl}$ (saturated solution, $70 \mathrm{~mL}$ ), and extracted with ether $(2 \square 100 \mathrm{~mL})$. The combined organic layers were washed with brine, dried over $\mathrm{MgSO}_{4}$, filtered, and concentrated in vacuo. The crude product was purified by flash chromatography on silica gel (elution with ether:hexane $=1: 25$ ) to give alkene $24(9.6 \mathrm{~g}, 82 \%)$. [ $[\square]_{\mathrm{D}}^{25}+2.6^{\circ},\left(c 6.2, \mathrm{CHCl}_{3}\right) ;{ }^{1} \mathrm{H} \mathrm{NMR}\left(500 \mathrm{MHz}, \mathrm{CDCl}_{3}\right) \square 0.87(\mathrm{~d}, 3 \mathrm{H}, J=6.6$ $\mathrm{Hz}), 1.20-1.27(\mathrm{~m}, 1 \mathrm{H}), 1.53-1.59(\mathrm{~m}, 1 \mathrm{H}), 1.77-1.84(\mathrm{~m}, 1 \mathrm{H}), 2.01-2.14(\mathrm{~m}, 2 \mathrm{H}), 3.25(\mathrm{dd}, 1 \mathrm{H}, J=9.0,6.6$ $\mathrm{Hz}), 3.32(\mathrm{dd}, 1 \mathrm{H}, J=9.0,6.1 \mathrm{~Hz}), 4.51(\mathrm{~s}, 2 \mathrm{H}), 4.93-5.04(\mathrm{~m}, 2 \mathrm{H}), 5.77-5.86(\mathrm{~m}, 1 \mathrm{H}), 7.25-7.35(\mathrm{~m}, 5 \mathrm{H})$; ${ }^{13} \mathrm{C}$ NMR $\left(125 \mathrm{MHz}, \mathrm{CDCl}_{3}\right) \square 16.9,31.1,32.8,32.9,72.9,75.7,114.2,127.4,127.5,128.2,138.7,139.0$; IR $\left(\mathrm{CHCl}_{3}\right) 2854,1640,1453,1362,1095,1028,994,907,732,696 \mathrm{~cm}^{-1}$; MS(CI) calculated for $\mathrm{C}_{14} \mathrm{H}_{20} \mathrm{O}$ $204.1\left(\mathrm{M}^{+}\right)$, found $205(\mathrm{M}+1)$. 


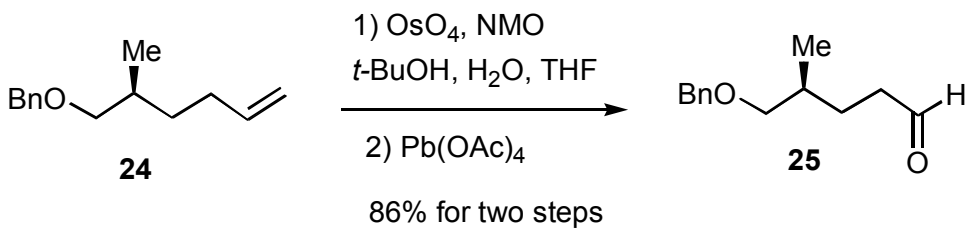

Aldehyde 25. A solution of alkene $24(9.6 \mathrm{~g}, 47 \mathrm{mmol})$ in THF (384 mL) was treated with $t$-BuOH (168 $\mathrm{mL})$, water $(75 \mathrm{~mL}), \mathrm{NMO}(13.8 \mathrm{~g}, 118 \mathrm{mmol}), \mathrm{OsO}_{4}(2.5 \mathrm{wt} \%$ in $t$-BuOH, $29.5 \mathrm{~mL}, 2.35 \mathrm{mmol})$. After being stirred for $16 \mathrm{~h}$ at room temperature, the reaction mixture was quenched with $\mathrm{Na}_{2} \mathrm{~S}_{2} \mathrm{O}_{3} \cdot 5 \mathrm{H}_{2} \mathrm{O}(30 \mathrm{~g})$ and water $(100 \mathrm{~mL})$. Stirring was continued for additional $2 \mathrm{~h}$ period. The organic layer was separated and washed with brine $(100 \mathrm{~mL})$, dried over $\mathrm{MgSO}_{4}$, filtered, and concentrated in vacuo to afford a corresponding diol, which was used directly in next step without further purification. $\mathrm{Pb}(\mathrm{OAc})_{4}(16.0 \mathrm{~g}, 36$ mmol) was added to the solution of the diol $(6.0 \mathrm{~g}, 25 \mathrm{mmol})$ in EtOAc $(500 \mathrm{~mL})$ at $0{ }^{\circ} \mathrm{C}$. The reaction mixture was stirred at $0{ }^{\circ} \mathrm{C}$ until TLC indicated complete consumption of starting material. After filtration through Celite, and concentration in vacuo, the crude product was purified by flash chromatography on silica gel (elution with ether:hexane $=1: 4)$ to give aldehyde $25(4.9 \mathrm{~g}, 86 \% \text { from alkene 24). [ }]^{25}{ }_{\mathrm{D}}-4.7^{\circ},(c$ 7.5, $\left.\mathrm{CHCl}_{3}\right) ;{ }^{1} \mathrm{H}$ NMR $\left(500 \mathrm{MHz}, \mathrm{CDCl}_{3}\right) \square 0.87$ (d, 3H, J=6.6 Hz), 1.38-1.52 (m, 1H), 1.70-1.77 (m, $\left.2 \mathrm{H}\right)$, 2.24-2.46 (m, 2H), 3.20-3.26 (m, 2H), $4.42(\mathrm{~s}, 2 \mathrm{H}), 7.25-7.35(\mathrm{~m}, 5 \mathrm{H}), 9.69(\mathrm{t}, 1 \mathrm{H}, J=2.0 \mathrm{~Hz}) ;{ }^{13} \mathrm{C} \mathrm{NMR}$ $\left(125 \mathrm{MHz}, \mathrm{CDCl}_{3}\right) \square 16.9,25.9,33.0,41.6,73.0,75.3,127.5,127.6,128.4,138.5,202.8 ; \mathrm{IR}\left(\mathrm{CHCl}_{3}\right) 2854$, $1722,1453,1092,1027,735,697 \mathrm{~cm}^{-1}$.

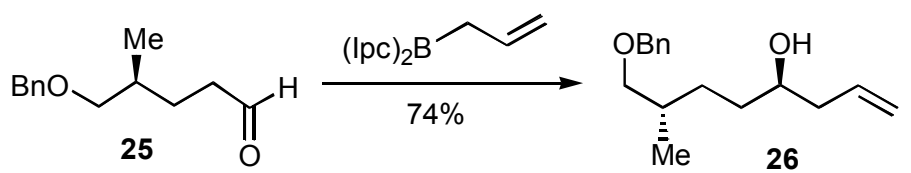

Alcohol 26. To a solution of (-)-MeOB(Ipc) $)_{2}(9.8 \mathrm{~g}, 31 \mathrm{mmol})$ in ether $(70 \mathrm{~mL})$ was added allyl magnesium bromide (1 M solution in ether, $28.6 \mathrm{~mL}, 28.6 \mathrm{mmol})$ at $0{ }^{\circ} \mathrm{C}$. The reacton mixture was stirred for $1 \mathrm{~h}$ at room temperature, and cooled to $-78{ }^{\circ} \mathrm{C}$. A solution of aldehyde 25 (4.9 g, $\left.23.8 \mathrm{mmol}\right)$ in ether $(55 \mathrm{~mL})$ was added. The resulting mixture was stirred for $3 \mathrm{~h}$ at $-78{ }^{\circ} \mathrm{C}$, quenched with $\mathrm{NaOH}(3 \mathrm{M}, 50$ $\mathrm{mL})$ and $\mathrm{H}_{2} \mathrm{O}_{2}(30 \mathrm{wt} \%, 50 \mathrm{~mL})$, and stirred for $12 \mathrm{~h}$. Layers were separated. The aqueous layer was extracted with EtOAc $(2 \square 100 \mathrm{~mL})$. Combined organic layers dried over $\mathrm{MgSO}_{4}$, filtered, and concentrated in vaсиo. The crude product was purified by flash chromatography on silica gel (elution with

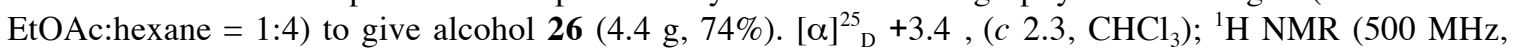
$\left.\mathrm{CDCl}_{3}\right) \square 0.94(\mathrm{~d}, 3 \mathrm{H}, J=6.7 \mathrm{~Hz}), 1.13-1.20(\mathrm{~m}, 1 \mathrm{H}), 1.39-1.46(\mathrm{~m}, 1 \mathrm{H}), 1.48-1.60(\mathrm{~m}, 2 \mathrm{H}), 1.74-1.81(\mathrm{~m}$, $1 \mathrm{H}), 2.10-2.16(\mathrm{~m}, 1 \mathrm{H}), 2.27-2.32(\mathrm{~m}, 1 \mathrm{H}), 3.26(\mathrm{dd}, 1 \mathrm{H}, J=9.0,6.5 \mathrm{~Hz}), 3.33(\mathrm{dd}, 1 \mathrm{H}, J=9.0,6.0 \mathrm{~Hz})$, 3.59-3.65 (m ,1H), $4.49(\mathrm{~s}, 2 \mathrm{H}), 5.10-5.15(\mathrm{~m}, 2 \mathrm{H}), 5.7-5.86(\mathrm{~m}, 1 \mathrm{H}), 7.25-7.35(\mathrm{~m}, 5 \mathrm{H}) ;{ }^{13} \mathrm{C}$ NMR $(125 \mathrm{M}$ $\left.\mathrm{Hz}, \mathrm{CDCl}_{3}\right) \square 17.1,29.5,33.4,34.0,41.7,70.9,72.9,75.6,118.0,127.4,127.5,128.2,134.8,138.6$; IR $\left(\mathrm{CHCl}_{3}\right)$ 3393, 2927, 1453, 1093, 1027, 910, 733, $696 \mathrm{~cm}^{-1}$; $\mathrm{MS}(\mathrm{CI})$ calculated for $\mathrm{C}_{16} \mathrm{H}_{24} \mathrm{O}_{2} 248.2\left(\mathrm{M}^{+}\right)$, found $249.1(\mathrm{M}+1)$.

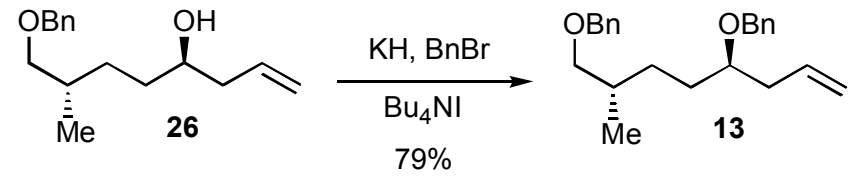

Alkene 13. A suspension of KH (1.05 g, $26.4 \mathrm{mmol})$ in THF (40 mL) was treated with a solution of alcohol $26(5.5 \mathrm{~g}, 22 \mathrm{mmol})$ in $\mathrm{THF}(20 \mathrm{~mL})$ at $0{ }^{\circ} \mathrm{C}$. After $20 \mathrm{~min}$, the mixture was treated with $\mathrm{BnBr}(3.0$ $\mathrm{mL}, 25 \mathrm{mmol})$ and $\mathrm{Bu}_{4} \mathrm{NI}(400 \mathrm{mg}, 1.1 \mathrm{mmol})$, and stirring was continued for $18 \mathrm{~h}$ at room temperature. The resulting mixture was quenched with $\mathrm{NH}_{4} \mathrm{Cl}$ (saturated solution, $20 \mathrm{~mL}$ ) and extracted with ether $(2 \square 30$ 
$\mathrm{mL}$ ). The combined organic layers were washed with brine, dried over $\mathrm{MgSO}_{4}$, filtered, and concentrated in vacuo. The crude product was purified by flash chromatography on silica gel (elution with ether:hexane = 1:10) to give benzyl ether $\mathbf{1 3}(5.85 \mathrm{~g}, 79 \%)$. [ []$_{\mathrm{D}}^{25}+10.7^{\circ},\left(c 1.35, \mathrm{CHCl}_{3}\right) ;{ }^{1} \mathrm{H} \mathrm{NMR}\left(500 \mathrm{MHz}, \mathrm{CDCl}_{3}\right) \square$ $0.93(\mathrm{~d}, 3 \mathrm{H}, J=6.7 \mathrm{~Hz}), 1.11-1.19(\mathrm{~m}, 1 \mathrm{H}), 1.49-1.61(\mathrm{~m}, 3 \mathrm{H}), 1.71-1.78(\mathrm{~m}, 1 \mathrm{H}), 2.32(\mathrm{t}, 2 \mathrm{H}, J=8.0 \mathrm{~Hz})$, $3.25(\mathrm{dd}, 1 \mathrm{H}, J=9.5,6.5 \mathrm{~Hz}), 3.33(\mathrm{dd}, 1 \mathrm{H}, J=9.0,6.0 \mathrm{~Hz}), 3.40-3.44(\mathrm{~m}, 1 \mathrm{H}), 4.48-4.57(\mathrm{~m}, 4 \mathrm{H}), 5.04-$ $5.11(\mathrm{~m}, 2 \mathrm{H}), 5.85(\mathrm{ddt}, 1 \mathrm{H}, J=17.0,10.0,7.0 \mathrm{~Hz}), 7.25-7.35(\mathrm{~m}, 10 \mathrm{H}) ;{ }^{13} \mathrm{C}$ NMR $\left(125 \mathrm{MHz}, \mathrm{CDCl}_{3}\right)$ $17.1,29.2,30.9,33.5,38.1,70.8,72.9,75.7,78.7,116.8,127.34,127.35,127.44,127.6,127.7,128.2$, 134.9, 138.7, 138.8; IR $\left(\mathrm{CHCl}_{3}\right)$ 2854, 1453, 1346, 1092, 1069, 1027, 910, 733, $695 \mathrm{~cm}^{-1}$; $\mathrm{MS}(\mathrm{CI})$ calculated for $\mathrm{C}_{23} \mathrm{H}_{30} \mathrm{O}_{2} 338.2\left(\mathrm{M}^{+}\right)$, found $339.1(\mathrm{M}+1)$.

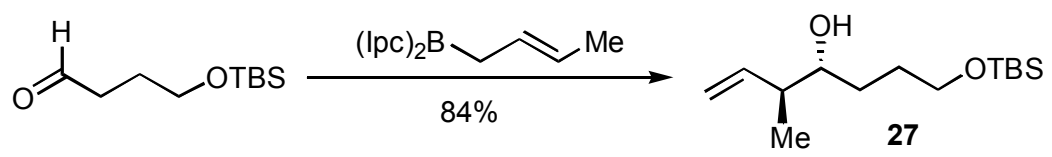

Alcohol 27. To a stirred suspension of $t$-BuOK $(1.06 \mathrm{~g}, 9.45 \mathrm{mmol})$ in THF $(20 \mathrm{~mL})$ were added trans 2butene $(2.2 \mathrm{~g}, 39.2 \mathrm{mmol})$ and $n$ - $\mathrm{BuLi}(2.5 \mathrm{M}$ in hexane, $3.78 \mathrm{~mL}, 9.45 \mathrm{mmol})$ at $-78{ }^{\circ} \mathrm{C}$. The reaction mixture was warmed up to $-45^{\circ} \mathrm{C}$, stirred for $10 \mathrm{~min}$, and cooled to $-78^{\circ} \mathrm{C}$. A solution of (-)-MeOB(Ipc) $)_{2}$ $(0.57 \mathrm{M}$ in THF, $16.6 \mathrm{~mL}, 9.46 \mathrm{mmol})$ was added. After $30 \mathrm{~min}, \mathrm{BF}_{3} \bullet \mathrm{OEt}_{2}(1.2 \mathrm{~mL}, 9.46 \mathrm{mmol})$ was added, followed by 4-siloxy butanal ${ }^{2}(1.59 \mathrm{~g}, 7.87 \mathrm{mmol})$. The resulting mixture was stirred for $2.5 \mathrm{~h}$ at $-78{ }^{\circ} \mathrm{C}$, quenched with $\mathrm{NaOH}(3 \mathrm{M}, 8 \mathrm{~mL})$ and $\mathrm{H}_{2} \mathrm{O}_{2}(30 \mathrm{wt} \%, 6 \mathrm{~mL})$, stirred for $12 \mathrm{~h}$, extracted with EtOAc $(2 \square 50 \mathrm{~mL})$, dried over $\mathrm{MgSO}_{4}$, filtered, and concentrated in vacuo. The crude product was purified by flash chromatography on silica gel (elution with EtOAc:hexane $=1: 4)$ to give alcohol $27(1.68 \mathrm{~g}, 84 \%$ yield, 99\% dr, 94\% ee). $[\square]_{\mathrm{D}}^{25}+3.3^{\circ}$, (neat); ${ }^{1} \mathrm{H}$ NMR (500 MHz, $\left.\mathrm{CDCl}_{3}\right) \square 0.05$ (s, 6H), 0.89 (s, 9H), 1.02 $(\mathrm{d}, 3 \mathrm{H}, J=6.9 \mathrm{~Hz}), 1.37-1.43(\mathrm{~m}, 1 \mathrm{H}), 1.60-1.68(\mathrm{~m}, 3 \mathrm{H}), 2.19-2.28(\mathrm{~m}, 1 \mathrm{H}), 2.42(\mathrm{br} \mathrm{s}, 1 \mathrm{H}), 3.41-3.45$ $(\mathrm{m}, 1 \mathrm{H}), 3.61-3.68(\mathrm{~m}, 2 \mathrm{H}), 5.05-5.09(\mathrm{~m}, 2 \mathrm{H}), 5.74-5.81(\mathrm{~m}, 1 \mathrm{H}) ;{ }^{13} \mathrm{C} \mathrm{NMR}\left(125 \mathrm{MHz}, \mathrm{CDCl}_{3}\right) \mathrm{-}-5.4$, 16.0, 18.2, 25.8, 29.1, 31.0, 43.9, 63.3, 74.4, 115.6, 140.5; $\mathrm{IR}_{\left(\mathrm{CHCl}_{3}\right)}$ 3421, 2929, 2857, 1471, 1255, 1094, $1004,911,832,733 \mathrm{~cm}^{-1}$; MS(CI) calculated for $\mathrm{C}_{14} \mathrm{H}_{30} \mathrm{O}_{2} \mathrm{Si} 258.2\left(\mathrm{M}^{+}\right)$, found $259(\mathrm{M}+1)$.

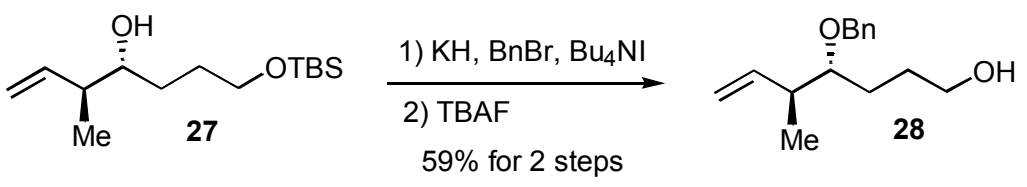

Alcohol 28. A solution of alcohol $27(16.0 \mathrm{~g}, 62 \mathrm{mmol})$ in THF $(60 \mathrm{~mL})$ was added to a suspension of $\mathrm{KH}$ $(3.0 \mathrm{~g}, 75 \mathrm{mmol})$ in THF $(140 \mathrm{~mL})$ at $0{ }^{\circ} \mathrm{C}$. After $20 \mathrm{~min}$, reaction mixture was treated with $\mathrm{BnBr}(8.4 \mathrm{~mL}$, $71 \mathrm{mmol})$ and $\mathrm{Bu}_{4} \mathrm{NI}(1.15 \mathrm{~g}, 3.1 \mathrm{mmol})$, stirred for $18 \mathrm{~h}$ at room temperature and quenched with $\mathrm{NH}_{4} \mathrm{Cl}$ (saturated solution, $60 \mathrm{~mL})$. Aqueous layer extracted with ether $(2 \square 80 \mathrm{ml})$. Combined organic layers were washed with brine, dried over $\mathrm{MgSO}_{4}$, filtered, and concentrated in vacuo. The crude product was dissolved in THF $(180 \mathrm{~mL})$. TBAF ( $1 \mathrm{M}$ solution in THF, $52 \mathrm{~mL}, 52 \mathrm{mmol}$ ) was added and the reaction mixture was stirred for $2.5 \mathrm{~h}$, washed with brine $(100 \mathrm{ml})$, dried over $\mathrm{MgSO}_{4}$, filtered, and concentrated in vacuo. The crude product was purified by flash chromatography on silica gel (elution with EtOAc:hexane $=2: 3$ ) to give alcohol 28 (8.5 g, 59\% for 2 steps). [ []$^{25}{ }_{\mathrm{D}}+0.82^{\circ},\left(c 7.8, \mathrm{CHCl}_{3}\right) ;{ }^{1} \mathrm{H} \mathrm{NMR}\left(500 \mathrm{MHz}, \mathrm{CDCl}_{3}\right) \square 1.03$ $(\mathrm{d}, 3 \mathrm{H}, J=6.9 \mathrm{~Hz}), 1.52-1.72(\mathrm{~m}, 4 \mathrm{H}), 1.76(\mathrm{br} \mathrm{s}, 1 \mathrm{H}), 2.56-2.61(\mathrm{~m}, 1 \mathrm{H}), 3.33(\mathrm{dt}, 1 \mathrm{H}, J=6.0,5.0 \mathrm{~Hz})$, $3.60(\mathrm{t}, 2 \mathrm{H}, J=6.0 \mathrm{~Hz}), 4.48(\mathrm{~d}, 1 \mathrm{H}, J=11.5 \mathrm{~Hz}), 4.59(\mathrm{~d}, 1 \mathrm{H}, J=11.5 \mathrm{~Hz}), 5.02-5.08(\mathrm{~m}, 2 \mathrm{H}), 5.81$ (ddd, $1 \mathrm{H}, J=17.0,10.0,7.0 \mathrm{~Hz}), 7.25-7.37(\mathrm{~m}, 5 \mathrm{H}) ;{ }^{13} \mathrm{C} \mathrm{NMR}\left(125 \mathrm{MHz}, \mathrm{CDCl}_{3}\right) \square 14.2,26.7,29.2,39.9,62.9$, 71.6, 82.6, 114.6, 127.5, 127.8, 128.3, 138.4, 140.8; IR $\left(\mathrm{CHCl}_{3}\right)$ 3349, 2867, 1453, 1059, 1027, 911, 733, $696 \mathrm{~cm}^{-1} ; \mathrm{MS}(\mathrm{CI})$ calculated for $\mathrm{C}_{15} \mathrm{H}_{22} \mathrm{O}_{2} 234.2\left(\mathrm{M}^{+}\right)$, found $235.1(\mathrm{M}+1)$. 


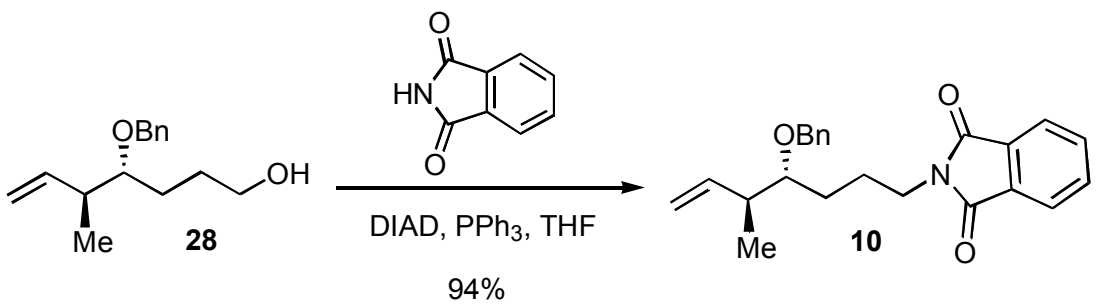

Alkene 10. A solution of alcohol 28 (5.5 g, $23.6 \mathrm{mmol})$ in THF (460 mL) was treated with $\mathrm{PPh}_{3}(6.3 \mathrm{~g}, 24$ mmol), phthalimide $(3.53 \mathrm{~g}, 24 \mathrm{mmol})$ and DIAD $(4.72 \mathrm{~mL}, 24 \mathrm{mmol})$. The reaction mixture was stirred for $18 \mathrm{~h}$ at room temperature, concentrated in vacuo and purified by flash chromatography on silica gel (elution with EtOAc:hexane = 1:4) to give alkene $10(8.0 \mathrm{~g}, 94 \%)$. [ $\left.]^{25}{ }_{\mathrm{D}}+100.3^{\circ},(c) 5.4, \mathrm{CHCl}_{3}\right)$; ${ }^{1} \mathrm{H} \mathrm{NMR}(500$ $\left.\mathrm{MHz}_{\mathrm{CDCl}}\right) \square 1.01(\mathrm{~d}, 3 \mathrm{H}, J=6.9 \mathrm{~Hz}), 1.42-1.58(\mathrm{~m}, 2 \mathrm{H}), 1.64-1.72(\mathrm{~m}, 1 \mathrm{H}), 1.79-1.87(\mathrm{~m}, 1 \mathrm{H}), 2.49-$ $2.53(\mathrm{~m}, 1 \mathrm{H}), 3.33(\mathrm{dt}, 1 \mathrm{H}, J=8.0,5.0 \mathrm{~Hz}), 3.61-3.69(\mathrm{~m}, 2 \mathrm{H}), 4.49(\mathrm{~d}, 1 \mathrm{H}, J=11.5 \mathrm{~Hz}), 4.55(\mathrm{~d}, 1 \mathrm{H}, J=$ $11.5 \mathrm{~Hz}), 4.98-5.04(\mathrm{~m}, 2 \mathrm{H}), 5.79(\mathrm{ddd}, 1 \mathrm{H}, J=17.0,10.0,7.0 \mathrm{~Hz}), 7.22-7.34(\mathrm{~m}, 5 \mathrm{H}), 7.69-7.71(\mathrm{~m}, 2 \mathrm{H})$, 7.81-7.84 (m, 2H); ${ }^{13} \mathrm{C}$ NMR (125 MHz, $\left.\mathrm{CDCl}_{3}\right) \square$ 14.5, 25.0, 27.8, 37.9, 40.2, 71.8, 82.1, 114.7, 123.1, 127.4, 127.7, 128.2, 132.1, 133.8, 133.9, 138.7, 140.6 ; IR $\left(\mathrm{CHCl}_{3}\right)$ 2937, 1707, 1394, 1362, 1066, 752, $718,696 \mathrm{~cm}^{-1}$; MS(CI) calculated for $\mathrm{C}_{23} \mathrm{H}_{25} \mathrm{NO}_{3} 363.2\left(\mathrm{M}^{+}\right)$, found $364.0(\mathrm{M}+1)$.

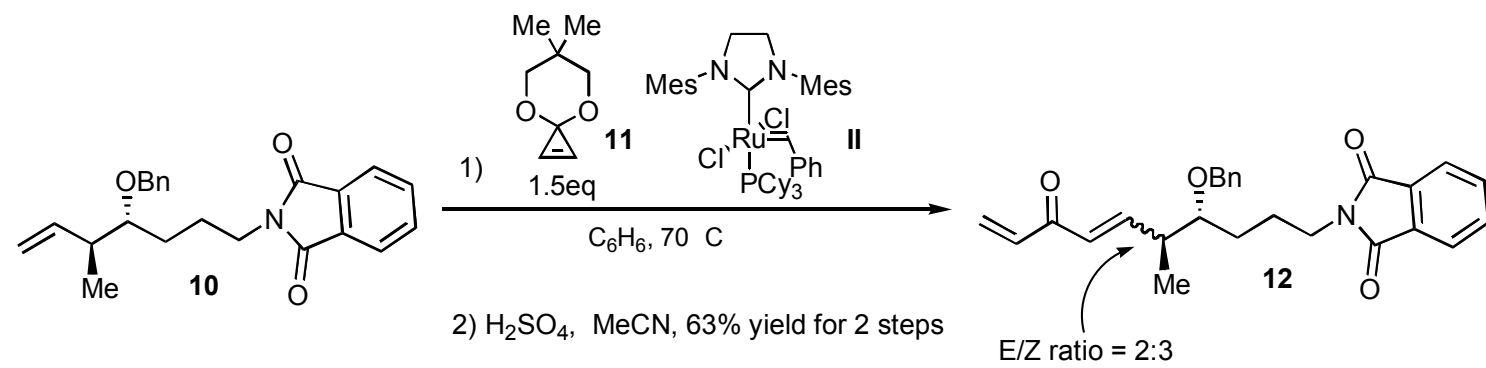

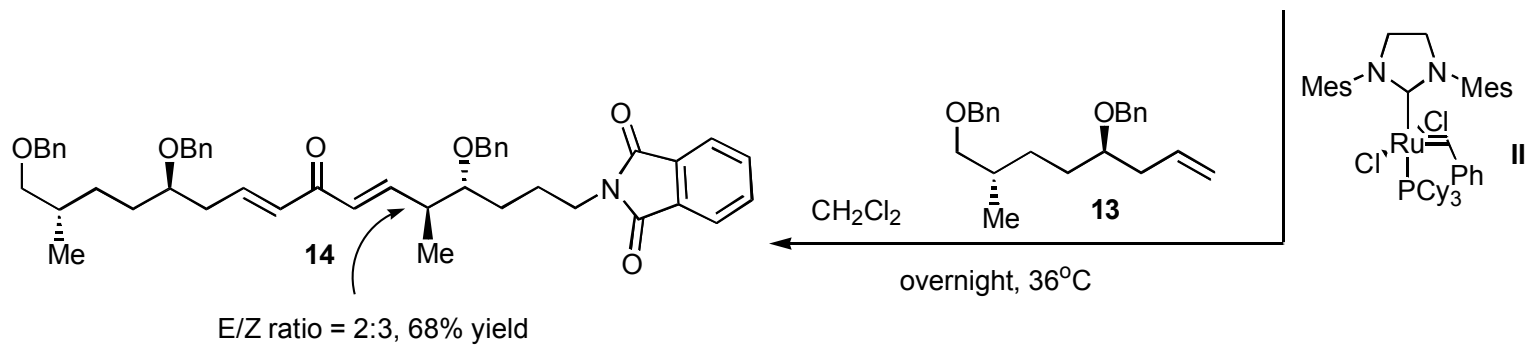

Dienone 14. The Grubbs catalyst II $(1.44 \mathrm{~g}, 1.7 \mathrm{mmol})$ was added to a solution of alkene 10 (6.0 g, 16.7 $\mathrm{mmol})$ and cyclopropenone acetal $\mathbf{1 1}^{2}(3.5 \mathrm{~g}, 25 \mathrm{mmol})$ in benzene $(135 \mathrm{~mL})$. The reaction mixture was stirred for $3 \mathrm{~h}$ at $70{ }^{\circ} \mathrm{C}$. Solvent was removed in vacuo and the residue $(5.68 \mathrm{~g})$ was dissolved in $\mathrm{CH}_{3} \mathrm{CN}$ $(320 \mathrm{~mL})$ and treated with $\mathrm{H}_{2} \mathrm{SO}_{4}(1 \mathrm{M}$ solution in water, $3 \mathrm{~mL}, 3 \mathrm{mmol})$ at $0{ }^{\circ} \mathrm{C}$. The reaction mixture was stirred for $1.5 \mathrm{~h}$ at $0{ }^{\circ} \mathrm{C}$ until TLC indicated complete consumption of the starting material, and diluted with water $(300 \mathrm{~mL})$. Extraction with ether $(500 \mathrm{~mL})$, drying over $\mathrm{MgSO}_{4}$, filtration and concentration in vacuo gave a crude product, which was purified by flash chromatography on silica gel (elution with EtOAc:hexane = 1:3) to give dienone $12(4.35 \mathrm{~g}, 72 \%$ yield) as a 2:3 mixture of cis and trans isomers which were carried to the next steps.

The Grubbs catalyst II ( $3 \mathrm{mg}, 0.004 \mathrm{mmol})$ was added to a solution of dienone 12 (26 $\mathrm{mg}, 0.073 \mathrm{mmol})$ and alkene $13(12 \mathrm{mg}, 0.037 \mathrm{mmol})$ in $\mathrm{CH}_{2} \mathrm{Cl}_{2}(0.3 \mathrm{~mL})$. The mixture was stirred for $10 \mathrm{~h}$ at $36{ }^{\circ} \mathrm{C}$. The solvent was removed in vacuo. The crude product was purified by flash chromatography on silica gel (elution with EtOAc:hexane = 10:1-> 4:1) to give dienone $14(17 \mathrm{mg}, 68 \%)$ as a mixture of trans:cis 
isomers as indicated above. Trans isomer, ${ }^{1} \mathrm{H}$ NMR $\left(500 \mathrm{MHz}, \mathrm{CDCl}_{3}\right) \square 0.92(\mathrm{~d}, 3 \mathrm{H}, J=6.7 \mathrm{~Hz}), 1.08(\mathrm{~d}$, $3 \mathrm{H}, J=6.8 \mathrm{~Hz}), 1.11-1.16(\mathrm{~m}, 1 \mathrm{H}), 1.43-1.48(\mathrm{~m}, 1 \mathrm{H}), 1.54-1.61(\mathrm{~m}, 4 \mathrm{H}), 1.68-1.75(\mathrm{~m}, 2 \mathrm{H}), 1.78-1.86(\mathrm{~m}$, $1 \mathrm{H}), 2.47(\mathrm{t}, 2 \mathrm{H}, J=6.5 \mathrm{~Hz}), 2.67(\mathrm{q}, 1 \mathrm{H}, J=6.5 \mathrm{~Hz}), 3.25(\mathrm{dd}, 1 \mathrm{H}, J=9.0,6.0 \mathrm{~Hz}), 3.30(\mathrm{dd}, 1 \mathrm{H}, J=9.0$, $7.0 \mathrm{~Hz}), 3.42(\mathrm{dt}, 1 \mathrm{H}, J=9.0,4.0 \mathrm{~Hz}), 3.49-3.53(\mathrm{~m}, 1 \mathrm{H}), 3.65(\mathrm{t}, 2 \mathrm{H}, J=6.5 \mathrm{~Hz}), 4.48-4.54(\mathrm{~m}, 6 \mathrm{H}), 6.31$ $(\mathrm{d}, 1 \mathrm{H}, J=16.0 \mathrm{~Hz}), 6.37(\mathrm{~d}, 1 \mathrm{H}, J=15.0 \mathrm{~Hz}), 6.87(\mathrm{dd}, 1 \mathrm{H}, J=15.5,7.5 \mathrm{~Hz}), 6.88-6.94(\mathrm{~m}, 1 \mathrm{H}), 7.24-$ $7.32(\mathrm{~m}, 15 \mathrm{H}), 7.69(\mathrm{dd}, 2 \mathrm{H}, J=5.0,3.0 \mathrm{~Hz}), 7.82(\mathrm{dd}, 2 \mathrm{H}, J=5.0,3.0 \mathrm{~Hz}){ }^{13} \mathrm{C} \mathrm{NMR}\left(125 \mathrm{MHz}^{\mathrm{CDCl}}{ }_{3}\right)$ $\square$ 15.0, 17.5, 25.0, 28.6, 29.5, 31.7, 33.8, 37.6, 38.1, 40.3, 71.5, 72.5, 73.3, 76.0, 78.5, 81.8, 123.5, 127.75, $127.83,127.91,127.95,128.1,128.2,128.6,128.7,128.9,129.2,130.9,132.4,134.2,138.6,138.8,139.0$, 144.4, 149.7, 168.7, 189.3; IR $\left(\mathrm{CHCl}_{3}\right)$ 1709, 1665, 1396, 1362, 1069, 735, $720,697 \mathrm{~cm}^{-1}$; MS(CI) calculated for $\mathrm{C}_{47} \mathrm{H}_{53} \mathrm{NO}_{6} 727.4\left(\mathrm{M}^{+}\right)$, found $728.2(\mathrm{M}+1)$.
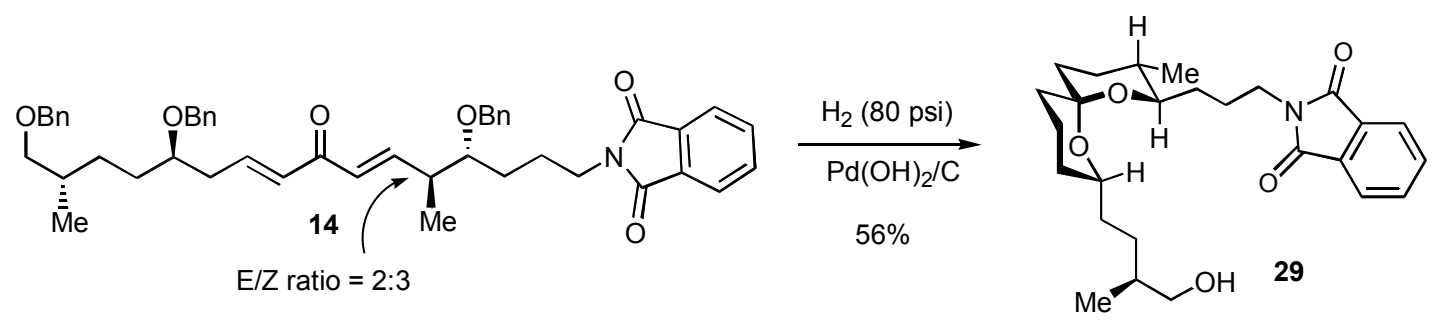

Spiroketal 29. A sealed tube was charged with dienone 14 (650 mg, $0.89 \mathrm{mmol}), \mathrm{Pd}(\mathrm{OH})_{2} / \mathrm{C}(640 \mathrm{mg}, 20$ wt\%, $0.91 \mathrm{mmol})$, EtOAc $(18 \mathrm{~mL})$ and $\mathrm{MeOH}(18 \mathrm{~mL})$. The reaction mixture was stirred for $4.5 \mathrm{~h}$ in $\mathrm{H}_{2}$ atmosphere (80 psi), filtered though Celite, and concentrated in vacuo. The crude product was purified by flash chromatography on silica gel (elution with EtOAc:hexane $=1: 1)$ to give alcohol $19(220 \mathrm{mg}, 56 \%)$. $[\square]_{\mathrm{D}}^{25}+32.4^{\circ},\left(c 1.14, \mathrm{CHCl}_{3}\right) ;{ }^{1} \mathrm{H}$ NMR $\left(500 \mathrm{MHz}, \mathrm{CDCl}_{3}\right) \square 0.70(\mathrm{~d}, 3 \mathrm{H}, J=6.5 \mathrm{~Hz}), 0.82(\mathrm{~d}, 3 \mathrm{H}, J=6.6$ $\mathrm{Hz}), 1.01-1.92(\mathrm{~m}, 20 \mathrm{H}), 3.08(\mathrm{dt}, 1 \mathrm{H}, J=9.8,1.4 \mathrm{~Hz}), 3.34(\mathrm{dd}, 1 \mathrm{H}, J=10.6,6.2 \mathrm{~Hz}), 3.38-3.42(\mathrm{~m}, 2 \mathrm{H})$, 3.56-3.67 (m, 2H), $7.60(\mathrm{dd}, 2 \mathrm{H}, J=5.4,3.0 \mathrm{~Hz}), 7.74(\mathrm{dd}, 2 \mathrm{H}, J=5.4,3.0 \mathrm{~Hz}) ;{ }^{13} \mathrm{C} \mathrm{NMR}(125 \mathrm{MHz}$, $\left.\mathrm{CDCl}_{3}\right) \square 17.0,18.3,19.3,25.3,28.1,29.4,30.7,31.5,33.7,35.3,35.7,36.0,36.3,38.6,68.5,69.6,74.3$, 95.8, 123.5, 132.4, 134.1, 168.8; $\mathrm{MS}(\mathrm{CI})$ calculated for $\mathrm{C}_{26} \mathrm{H}_{37} \mathrm{NO}_{5} 443.3\left(\mathrm{M}^{+}\right)$, found $444.1(\mathrm{M}+1)$.

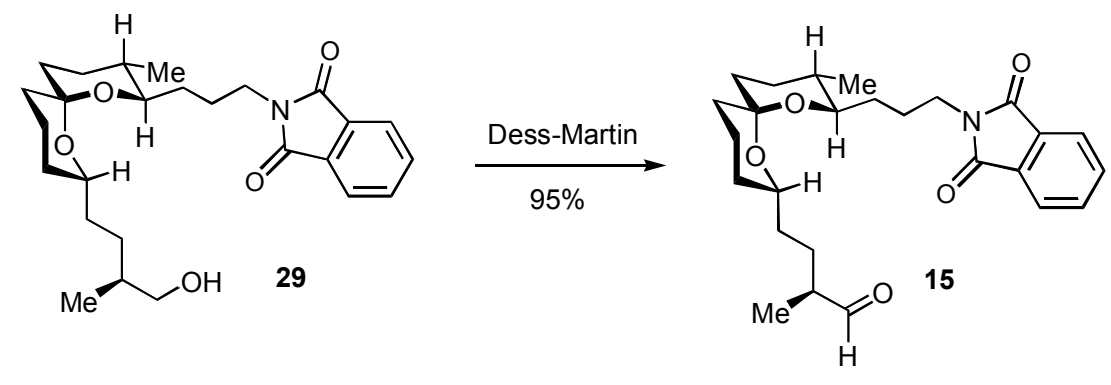

Aldehyde 15. A solution of alcohol $29(40 \mathrm{mg}, 0.09 \mathrm{mmol})$ in $\mathrm{CH}_{2} \mathrm{Cl}_{2}(6 \mathrm{~mL})$ was stirred with DessMartin reagent $(115 \mathrm{mg}, 0.27 \mathrm{mmol})$ for $1 \mathrm{~h}$ at room temperature. The reaction mixture was quenched with $\mathrm{NaHCO}_{3}$ (saturated solution, $5 \mathrm{~mL}$ ), extracted with $\mathrm{CH}_{2} \mathrm{Cl}_{2}(2 \square 10 \mathrm{~mL})$ and dried over $\mathrm{MgSO}_{4}$, filtered, and concentrated in vacuo. The crude product was purified by flash chromatography on silica gel (elution with ether:hexane $=1: 1)$ to give aldehyde $15(38 \mathrm{mg}, 95 \%)$. [ $]^{25}{ }_{\mathrm{D}}+41.5^{\circ},\left(c 2.3, \mathrm{CHCl}_{3}\right) ;{ }^{1} \mathrm{H} \mathrm{NMR}(500 \mathrm{MHz}$, $\left.\mathrm{CDCl}_{3}\right) \square 0.80(\mathrm{~d}, 3 \mathrm{H}, J=6.6 \mathrm{~Hz}), 1.08(\mathrm{~d}, 3 \mathrm{H}, J=7.0 \mathrm{~Hz}), 1.12-1.18(\mathrm{~m}, 1 \mathrm{H}), 1.21-1.72(\mathrm{~m}, 15 \mathrm{H}), 1.78-$ $1.89(\mathrm{~m} 1 \mathrm{H}), 1.93-2.02(\mathrm{~m}, 2 \mathrm{H}), 2.32-2.38(\mathrm{~m}, 1 \mathrm{H}), 3.13(\mathrm{dt}, 1 \mathrm{H}, J=10.0,2.0 \mathrm{~Hz}), 3.47-3.52(\mathrm{~m}, 1 \mathrm{H})$, $3.66-3.76(\mathrm{~m}, 2 \mathrm{H}), 7.70(\mathrm{dd}, 2 \mathrm{H}, J=6.0,3.0 \mathrm{~Hz}), 7.83(\mathrm{dd}, 2 \mathrm{H}, J=5.0,3.0 \mathrm{~Hz}), 9.61(\mathrm{~d}, 1 \mathrm{H}, J=2.0$ $\mathrm{Hz}) ;{ }^{13} \mathrm{C}$ NMR $\left(125 \mathrm{MHz}, \mathrm{CDCl}_{3}\right) \square 13.1,18.0,18.9,25.1,26.6,27.8,30.5,31.1,33.5,34.9,35.3,35.9$, $38.3,46.3,69.0,74.1,95.4,123.1,132.1,133.8,168.4,205.2$. 

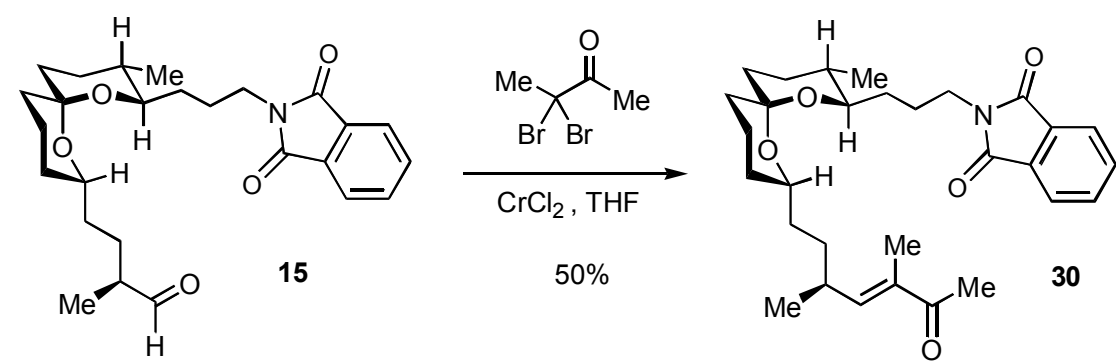

Ketone 30. A solution of aldehyde $15(39 \mathrm{mg}, 0.088 \mathrm{mmol})$ and dibromoketone $(21 \mathrm{mg}, 0.091 \mathrm{mmol})$ in THF $(1.5 \mathrm{ml})$ was added to a suspension of $\mathrm{CrCl}_{2}(49 \mathrm{mg}, 0.4 \mathrm{mmol})$ in THF $(1.5 \mathrm{~mL})$ via canula. Reaction mixture was stirred for $40 \mathrm{~min}$, quenched with water $(4 \mathrm{~mL})$, extracted with ether $(2 \square 10 \mathrm{~mL})$, dried over $\mathrm{MgSO}_{4}$, filtered, and concentrated in vacuo. The crude product was purified by flash chromatography on silica gel (elution with ether:hexane $=1: 4)$ to give enone $30(22 \mathrm{mg}, 50 \%)$. [ $]^{25}{ }_{\mathrm{D}}+23.2^{\circ},\left(c 0.64, \mathrm{CHCl}_{3}\right)$; ${ }^{1} \mathrm{H}$ NMR $\left(500 \mathrm{MHz}, \mathrm{CDCl}_{3}\right) \square 0.81(\mathrm{~d}, 3 \mathrm{H}, J=6.4 \mathrm{~Hz}), 1.03(\mathrm{~d}, 3 \mathrm{H}, J=6.6 \mathrm{~Hz}), 1.02-1.17(\mathrm{~m}, 1 \mathrm{H}), 1.27-$ $1.61(\mathrm{~m}, 14 \mathrm{H}), 1.63-1.72(\mathrm{~m}, 2 \mathrm{H}), 1.74(\mathrm{~d}, 3 \mathrm{H}, J=1.0 \mathrm{~Hz}), 1.76-1.88(\mathrm{~m}, 1 \mathrm{H}), 1.93-2.05(\mathrm{~m}, 1 \mathrm{H}), 2.30,(\mathrm{~s}$, $3 \mathrm{H}), 2.51-2.59(\mathrm{~m}, 1 \mathrm{H}), 3.15(\mathrm{dt}, 1 \mathrm{H}, J=10.0,2.0 \mathrm{~Hz}), 3.45-3.50(\mathrm{~m}, 1 \mathrm{H}), 3.66-3.77(\mathrm{~m}, 2 \mathrm{H}), 6.39(\mathrm{dd}, 1 \mathrm{H}$, $J=10.0,1.0 \mathrm{~Hz}), 7.71(\mathrm{dd}, 2 \mathrm{H}, J=5.0,3.0 \mathrm{~Hz}), 7.83(\mathrm{dd}, 2 \mathrm{H}, J=5.5,3.0 \mathrm{~Hz}) ;{ }^{13} \mathrm{C} \mathrm{NMR}(125 \mathrm{MHz}$, $\left.\mathrm{CDCl}_{3}\right) \square 11.6,18.3,19.3,20.3,25.5,25.9,28.1,30.9,31.6,33.2,34.0,34.5,35.3,35.7,36.3,38.6,69.2$, 74.4, 95.7, 123.5, 132.5, 134.1, 136.5, 149.8, 168.7, 200.6; IR $\left(\mathrm{CHCl}_{3}\right)$ 2931, 1714, 1668, 1396, 1368, 985, $757,720 \mathrm{~cm}^{-1}$; $\mathrm{MS}(\mathrm{CI})$ calculated for $\mathrm{C}_{30} \mathrm{H}_{41} \mathrm{NO}_{5} 495.3\left(\mathrm{M}^{+}\right)$, found $496.2(\mathrm{M}+1)$.
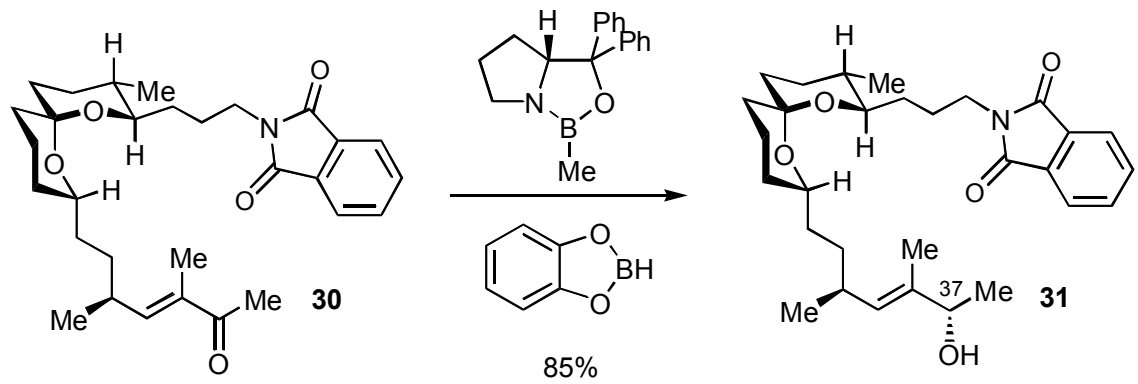

(37S)-Alcohol 31. To a solution of enone $30(13 \mathrm{mg}, 0.024 \mathrm{mmol})$ and $(R)-2$-methyl-CBS-oxazoborolidine ( $1 \mathrm{M}$ in toluene, $26 \square \mathrm{L}, 0.026 \mathrm{mmol})$ in toluene $(0.4 \mathrm{~mL})$ was added catechol borane $(5.1 \square \mathrm{L}, 0.048 \mathrm{mmol})$ in toluene $(0.1 \mathrm{~mL})$ at $-78{ }^{\circ} \mathrm{C}$. The reaction mixture was stirred for $12 \mathrm{~h}$ at $-78{ }^{\circ} \mathrm{C}$, quenched with $\mathrm{MeOH}$ $(50 \square \mathrm{L})$. After stirring for $4 \mathrm{~h}$ at room temperature, EtOAc $(10 \mathrm{~mL})$ was added. The solution was washed with $\mathrm{Na}_{2} \mathrm{CO}_{3}$ (saturated solution, $2 \square 5 \mathrm{~mL}$ ), dried over $\mathrm{MgSO}_{4}$, filtered, and concentrated in vacuo. The crude product was purified by flash chromatography on silica gel (elution with ether:hexane $=1: 1$ ) to give alcohol 31 (11 mg, 85\%). [ $]_{\mathrm{D}}^{25}+23.8^{\circ},\left(\mathrm{c} 0.77, \mathrm{CHCl}_{3}\right) ;{ }^{1} \mathrm{H} \mathrm{NMR}\left(500 \mathrm{MHz}, \mathrm{CDCl}_{3}\right) \square 0.80(\mathrm{~d}, 3 \mathrm{H}, J=$ $6.5 \mathrm{~Hz}), 0.93(\mathrm{~d}, 3 \mathrm{H}, J=6.7 \mathrm{~Hz}), 1.08-1.17(\mathrm{~m}, 1 \mathrm{H}), 1.23(\mathrm{~d}, 3 \mathrm{H}, J=6.4 \mathrm{~Hz}), 1.27-1.58(\mathrm{~m}, 15 \mathrm{H}), 1.59(\mathrm{~s}$, $3 \mathrm{H}), 1.63-1.74(\mathrm{~m}, 2 \mathrm{H}), 1.77-1.88(\mathrm{~m}, 1 \mathrm{H}), 1.95-2.05(\mathrm{~m}, 1 \mathrm{H}), 2.30-2.40(\mathrm{~m}, 1 \mathrm{H}), 3.13-3.18(\mathrm{~m}, 1 \mathrm{H}), 3.40-$ $3.48(\mathrm{~m}, 1 \mathrm{H}), 3.65-3.77(\mathrm{~m}, 2 \mathrm{H}), 4.15-4.20(\mathrm{~m}, 1 \mathrm{H}), 5.17(\mathrm{~d}, 1 \mathrm{H}, J=9.2 \mathrm{~Hz}), 7.71(\mathrm{dd}, 2 \mathrm{H}, J=5.3,3.0$ $\mathrm{Hz}), 7.85(\mathrm{dd}, 2 \mathrm{H}, J=5.3,3.1 \mathrm{~Hz}){ }^{13} \mathrm{C} \mathrm{NMR}\left(125 \mathrm{MHz}, \mathrm{CDCl}_{3}\right) \square 11.6,18.0,19.1,20.9,21.6,25.2,27.8$, 30.6, 31.3, 31.9, 33.5, 34.1, 35.0, 35.4, 36.0, 38.3, 69.1, 73.4, 74.0, 95.4, 123.2, 131.6, 132.2, 133.8, 137.1, 168.5; IR $\left(\mathrm{CHCl}_{3}\right)$ 2927, 1712, 1456, 1396, 1367, 1073, 984, $721 \mathrm{~cm}^{-1}$; MS(API-ES) calculated for $\mathrm{C}_{30} \mathrm{H}_{43} \mathrm{NO}_{5} 497.3\left(\mathrm{M}^{+}\right)$, found $520.2(\mathrm{M}+\mathrm{Na})$. 

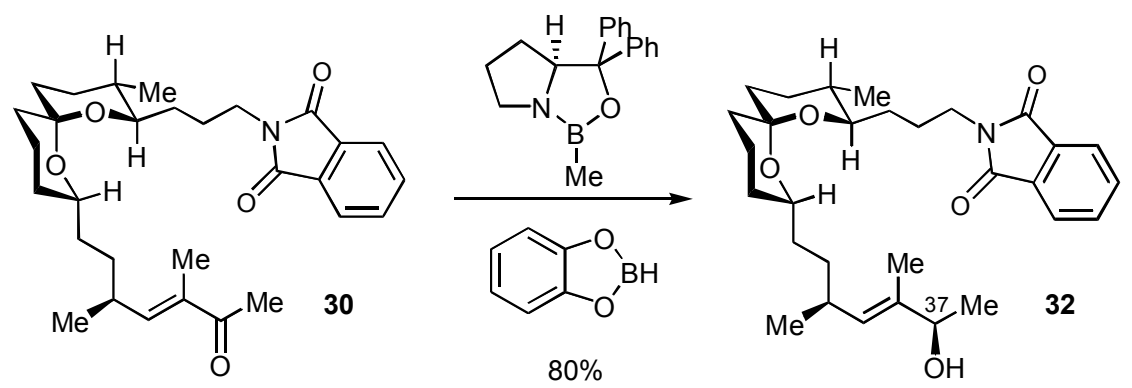

(37R)-Alcohol 32. Alcohol 32 was prepared according to the procedure used for the synthesis of diastereomeric alcohol 31. [ $]_{\mathrm{D}}^{25}+23.1^{\circ},\left(c\right.$ 1.8, $\left.\mathrm{CHCl}_{3}\right) ;{ }^{1} \mathrm{H} \mathrm{NMR}\left(500 \mathrm{MHz}, \mathrm{CDCl}_{3}\right) \square 0.81(\mathrm{~d}, 3 \mathrm{H}, J=6.5$ $\mathrm{Hz}), 0.92(\mathrm{~d}, 3 \mathrm{H}, J=6.7 \mathrm{~Hz}), 1.03-1.17(\mathrm{~m}, 1 \mathrm{H}), 1.24(\mathrm{~d}, 3 \mathrm{H}, J=6.4 \mathrm{~Hz}), 1.27-1.58(\mathrm{~m}, 15 \mathrm{H}), 1.60(\mathrm{~s}, 3 \mathrm{H})$, 1.63-1.74 (m, 2H), 1.77-1.88 (m, 1H), 1.95-2.05 (m, 1H), 2.30-2.38 (m, 1H), 3.16-3.22 (m, 1H), 3.43-3.49 $(\mathrm{m}, 1 \mathrm{H}), 3.67-3.79(\mathrm{~m}, 2 \mathrm{H}), 4.18(\mathrm{q}, 1 \mathrm{H}, J=6.0 \mathrm{~Hz}), 5.17(\mathrm{~d}, 1 \mathrm{H}, J=9.0 \mathrm{~Hz}), 7.71(\mathrm{dd}, 2 \mathrm{H}, J=6.0,3.0$ $\mathrm{Hz}), 7.85(\mathrm{dd}, 2 \mathrm{H}, J=6.0,3.0 \mathrm{~Hz}){ }^{13} \mathrm{C} \mathrm{NMR}\left(125 \mathrm{MHz}, \mathrm{CDCl}_{3}\right) \square 11.6,18.0,19.2,20.8,21.5,25.0,27.9$, $30.5,31.3,31.9$, 33.5, 34.1, 35.0, 35.4, 36.1, 38.3, 69.2, 73.5, 73.9, 95.4, 123.2, 131.6, 132.2, 133.9, 137.1, 168.5; IR $\left(\mathrm{CHCl}_{3}\right) 3469,2932$, , 2868, 1772, 1714, 1456, 1396, 1368, 1093, 984, $721 \mathrm{~cm}^{-1}$.

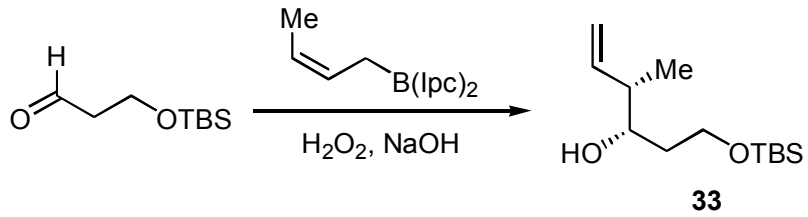

Alcohol 33. To a stirred suspension of $t$-BuOK $(6.4 \mathrm{~g}, 57 \mathrm{mmol})$ in THF $(132 \mathrm{~mL})$ were added cis 2butene $(14.52 \mathrm{~g}, 259 \mathrm{mmol})$ and $n$-BuLi $(1.6 \mathrm{M}$ in hexanes, $35.64 \mathrm{~mL}, 57 \mathrm{mmol})$ at $-78{ }^{\circ} \mathrm{C}$. The solution was warmed up to $-45^{\circ} \mathrm{C}$, stirred for $10 \mathrm{~min}$, cooled back to $-78{ }^{\circ} \mathrm{C}$. Solution of (+)-(Ipc) $)_{2} \mathrm{BOMe}(18 \mathrm{~g}, 57$ $\mathrm{mmol})$ in $57 \mathrm{ml}$ of $\mathrm{THF}$ was added dropwise to the flask at $-78{ }^{\circ} \mathrm{C}$. After $30 \mathrm{~min}, \mathrm{BF}_{3} \bullet \mathrm{OEt}_{2}(7.23 \mathrm{~mL}, 57$ mmol) was added, followed by 3-siloxy $\operatorname{propanal}^{3}(9.77 \mathrm{~g}, 52 \mathrm{mmol})$. The resulting mixture was stirred for $3 \mathrm{~h}$ at $-78{ }^{\circ} \mathrm{C}$, and quenched with $\mathrm{NaOH}(3 \mathrm{M}, 60 \mathrm{~mL})$ and $\mathrm{H}_{2} \mathrm{O}_{2}(30 \mathrm{wt} \%, 40 \mathrm{~mL})$, stirred overnight. Aqueous phase was extracted with ethyl acetate $(3 \square 200 \mathrm{ml})$, dried with $\mathrm{MgSO}_{4}$, filtered, and concentrated in vacuo. The crude product was purified by flash chromatography on silica gel (elution with EtOAc:hexane = 1:9) to give alcohol 33 (8.04 g, $63 \%$ yield, 93\% ee - determined by Mosher ester

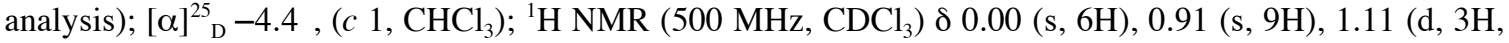
$\mathrm{J}=6.8 \mathrm{~Hz}), 1.56(\mathrm{~m}, 2 \mathrm{H}), 2.23(\mathrm{~m}, 1 \mathrm{H}), 2.81(\mathrm{~d}, 1 \mathrm{H}, \mathrm{J}=2.9 \mathrm{~Hz}), 3.60(\mathrm{~m}, 2 \mathrm{H}), 3.71(\mathrm{~m}, 1 \mathrm{H}), 4.98(\mathrm{dd}, 2 \mathrm{H}$, $J=16.0,8.4 \mathrm{~Hz}), 5.77(\mathrm{ddd}, 1 \mathrm{H}, J=7.8,10.1,17.5 \mathrm{~Hz}) ;{ }^{13} \mathrm{C} \mathrm{NMR}\left(125 \mathrm{MHz}, \mathrm{CDCl}_{3}\right) \square-5.6,15.1,25.8$, 35.4, 43.9, 62.9, 75.3, 114.7, 141.1; IR (neat) 3440, 2954, 2928, 2857, 1471, 1255, 1081, 833, $774 \mathrm{~cm}^{-1}$; MS calculated for $\mathrm{C}_{13} \mathrm{H}_{28} \mathrm{O}_{2} \mathrm{Si}(\mathrm{M}+) 244.45$, found $245(\mathrm{M}+)$.

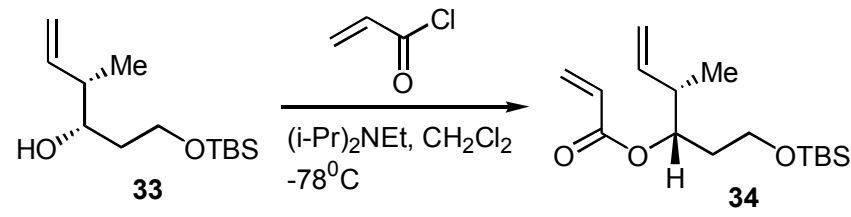

Diene 34. A solution of alcohol $33(7.82 \mathrm{~g}, 32 \mathrm{mmol})$ in $400 \mathrm{ml}$ of dry $\mathrm{CH}_{2} \mathrm{Cl}_{2}$ was treated with $\mathrm{EtN}(\mathrm{iPr})_{2}$ $(16.7 \mathrm{ml}, 96 \mathrm{mmol})$ and DMAP $(0.782 \mathrm{~g}, 6.04 \mathrm{mmol})$. Reaction mixture was cooled to $-78{ }^{\circ} \mathrm{C}$, and treated dropwise with acryloyl chloride $(5.72 \mathrm{ml}, 70.4 \mathrm{mmol})$. The resulting mixture was warmed to room 
temperature, stirred for $3 \mathrm{~h}$, partially concentrated and filtered through a plug of silica gel deactivated with $\mathrm{NEt}_{3}$. Silica gel was washed with additional amount of $\mathrm{CH}_{2} \mathrm{Cl}_{2}(100 \mathrm{ml})$. All filtrates were collected and concentrated in vacuo. The crude product was purified by flash chromatography on silica gel (elution with $\mathrm{NEt}_{3}:$ EtOAc:Hex = 1:10:100) to give diene $34(8.4 \mathrm{~g}, 88 \%$ yield $)$; [ $]^{25}{ }_{\mathrm{D}}-29.1^{\circ},\left(\mathrm{c} 1.6, \mathrm{CHCl}_{3}\right)$; ${ }^{1} \mathrm{H} \mathrm{NMR}$ $\left(500 \mathrm{MHz}, \mathrm{CDCl}_{3}\right) \square 0.04(\mathrm{~s}, 6 \mathrm{H}), 0.88(\mathrm{~s}, 9 \mathrm{H}), 1.02(\mathrm{~d}, 3 \mathrm{H}, J=6.9 \mathrm{~Hz}), 1.81(\mathrm{~m}, 2 \mathrm{H}), 2.52(\mathrm{~m}, 1 \mathrm{H}), 3.64$ $(\mathrm{m}, 2 \mathrm{H}), 5.04(\mathrm{~m}, 3 \mathrm{H}), 5.81(\mathrm{~m}, 2 \mathrm{H}), 6.12(\mathrm{dd}, 1 \mathrm{H}, J=10,17 \mathrm{~Hz}), 6.40(\mathrm{dd}, 1 \mathrm{H}, J=1,17 \mathrm{~Hz}) ;{ }^{13} \mathrm{C}$ NMR $(125$ $\left.\mathrm{MHz}, \mathrm{CDCl}_{3}\right) \square-5.5,15.1,25.8,34.2,41.3,59.5,74.2,115.3,128.6,130.3,139.4,165.6$; IR (neat) 2927, 1724, 1472, 1404, 1256, 1190, 1094, 1045, 983, 916, 833, 807, 773, $662 \mathrm{~cm}^{-1}$; MS calculated for $\mathrm{C}_{16} \mathrm{H}_{30} \mathrm{O}_{3} \mathrm{Si}(\mathrm{M}+)$ 298.51, found $299(\mathrm{M}+)$.
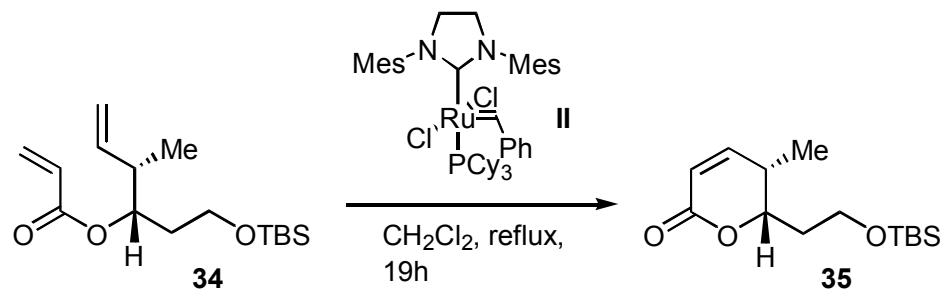

Lactone 35. A solution of diene $34(2.5 \mathrm{~g}, 8.4 \mathrm{mmol})$ in $75 \mathrm{ml}$ of dry $\mathrm{CH}_{2} \mathrm{Cl}_{2}$ was treated with the Grubbs catalyst II (0.975 g, $1.14 \mathrm{mmol})$. The resulting brown solution was heated under mild reflux for $19 \mathrm{~h}$. The reaction mixture was then concentrated in vacuo. The crude product was purified by flash chromatography on silica gel (elution with EtOAc:Hex = 1:9 -> 1:4) to give lactone $35(1.65 \mathrm{~g}, 72 \% \text { yield); [ }]^{25}{ }_{\mathrm{D}}+29.1^{\circ},(c$ 1.9, $\mathrm{CHCl}_{3}$ ); ${ }^{1} \mathrm{H}$ NMR $\left(500 \mathrm{MHz}, \mathrm{CDCl}_{3}\right) \square 0.05$ (s, 6H), 0.88 (s, 9H), 1.05 (d, 3H, J=7.1 Hz), 1.75 (m, $\left.1 \mathrm{H}\right)$, $1.97(\mathrm{~m}, 1 \mathrm{H}), 2.39(\mathrm{~m}, 1 \mathrm{H}), 3.80(\mathrm{~m}, 2 \mathrm{H}), 4.63(\mathrm{dt}, 1 \mathrm{H}, J=3.9,9.1 \mathrm{~Hz}), 5.97(\mathrm{~d}, 1 \mathrm{H}, J=9.6 \mathrm{~Hz}), 6.97(\mathrm{dd}$, $1 \mathrm{H}, J=6.2 \mathrm{~Hz}, 9.7 \mathrm{~Hz}) ;{ }^{13} \mathrm{C} \mathrm{NMR}\left(125 \mathrm{MHz}, \mathrm{CDCl}_{3}\right) \square-5.5,11.4,18.2,25.8,32.2,34.5,58.7,76.8,119.8$, 151.6, 164.4; IR (neat) 2927, 1721, 1471, 1380, 1248, 1089, 986, 938, 824, 775, 752, $664 \mathrm{~cm}^{-1}$; MS calculated for $\mathrm{C}_{14} \mathrm{H}_{26} \mathrm{O}_{3} \mathrm{Si}(\mathrm{M}+) 270.17$, found $271(\mathrm{M}+)$.

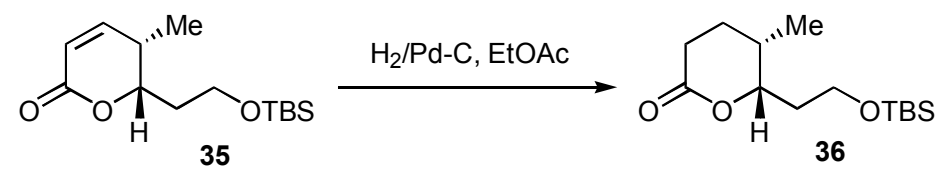

Lactone 36. A solution of lactone $35(2 \mathrm{~g}, 7.4 \mathrm{mmol})$ in $100 \mathrm{ml}$ of EtOAc was treated with $\mathrm{Pd} / \mathrm{C}(100 \mathrm{mg}$, $10 \%$ wt.) and the resulting mixture was stirred in $\mathrm{H}_{2}$ atmosphere $(1 \mathrm{~atm})$ for $3 \mathrm{~h}$. The reaction mixture was filtered and concentrated in vасио to give saturated lactone 36 (1.65 g, 100\% yield), which was determined to be spectroscopically pure. [ $\square]_{\mathrm{D}}^{25}-71^{\circ},\left(c 5.7, \mathrm{CHCl}_{3}\right) ;{ }^{1} \mathrm{H} \mathrm{NMR}\left(500 \mathrm{MHz}, \mathrm{CDCl}_{3}\right) \square 0.06(\mathrm{~s}, 6 \mathrm{H}), 0.87$ $(\mathrm{s}, 9 \mathrm{H}), 0.96(\mathrm{~d}, 3 \mathrm{H}, J=6.8 \mathrm{~Hz}), 1.68(\mathrm{~m}, 2 \mathrm{H}), 1.84(\mathrm{~m}, 1 \mathrm{H}), 2.05(\mathrm{~m}, 2 \mathrm{H}), 2.53(\mathrm{t}, 2 \mathrm{H}, J=7 \mathrm{~Hz}), 3.75(\mathrm{~m}$, 2H), $4.51(\mathrm{dt}, 1 \mathrm{H}, J=3,10 \mathrm{~Hz}) ;{ }^{13} \mathrm{C} \mathrm{NMR}\left(125 \mathrm{MHz}, \mathrm{CDCl}_{3}\right) \square 171.8,79.2,58.9,35.0,29.4,26.7,26.1$, 25.8, 18.2, 12.7, -5.5; IR $\left(\mathrm{CCl}_{4}\right)$ 2954, 1741, 1471, 1388, 1360, 1250, 1074, 836, 779, $753 \mathrm{~cm}^{-1}$; MS calculated for $\mathrm{C}_{14} \mathrm{H}_{28} \mathrm{O}_{3} \mathrm{Si}(\mathrm{M}+)$ 272.5, found $273.1(\mathrm{M}+)$.

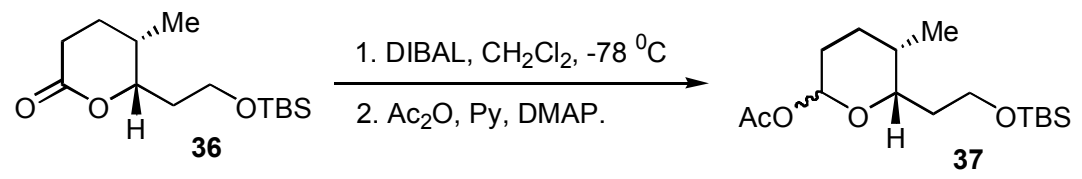

Acetate 37. A solution of lactone $36(1.65 \mathrm{~g}, 6.06 \mathrm{mmol})$ in $150 \mathrm{ml}$ of $\mathrm{CH}_{2} \mathrm{Cl}_{2}$ was treated with DIBAL $\left(1 \mathrm{M}\right.$ in $\left.\mathrm{CH}_{2} \mathrm{Cl}_{2}, 6.66 \mathrm{ml}, 6.66 \mathrm{mmol}\right)$ at $-78{ }^{\circ} \mathrm{C}$. The reaction mixture was stirred for $40 \mathrm{~min}$ at $-78^{\circ} \mathrm{C}$ and then quenched with $\mathrm{H}_{2} \mathrm{O}(80 \mathrm{ml})$, followed by $\mathrm{HCl}(1 \mathrm{M}, 12 \mathrm{ml})$. Organic layer was separated, dried over $\mathrm{MgSO}_{4}$, filtered and concentrated in vacuo to give pure lactol as a mixture of diastereomers $(1.4 \mathrm{~g}, 85 \%$ yield), which was used for the next step without purification. 
A solution of lactol $(1.4 \mathrm{~g}, 5.18 \mathrm{mmol})$ in $120 \mathrm{ml}$ of $\mathrm{CH}_{2} \mathrm{Cl}_{2}$ was treated with DMAP (176 mg, $\left.1.4 \mathrm{mmol}\right)$, pyridine $(1.64 \mathrm{ml}, 23 \mathrm{mmol})$ and $\mathrm{Ac}_{2} \mathrm{O}(1.44 \mathrm{ml}, 15.26 \mathrm{mmol})$. The reaction mixture was stirred for $3 \mathrm{~h}$ at room temperature and then quenched with $\mathrm{NaHCO}_{3}$ (saturated solution, $20 \mathrm{ml}$ ). Organic layer was separated and dried over $\mathrm{Na}_{2} \mathrm{SO}_{4}$, filtered and concentrated in vacuo. The crude product was purified by flash chromatography on silica gel (elution with EtOAc:Hex = 1:7) to give lactol acetate $37(1.31 \mathrm{~g}, 81 \%$ yield over 2 steps); [ []$^{25}{ }_{\mathrm{D}}^{2}-21^{\circ},\left(c 2.2, \mathrm{CH}_{2} \mathrm{Cl}_{2}\right) ;{ }^{1} \mathrm{H}$ NMR (500 MHz, $\left.\mathrm{CDCl}_{3}\right) \square 0.04$ (s, 3H), 0.05 (s, 3H), $0.89(\mathrm{~s}, 9 \mathrm{H}), 0.96(\mathrm{~d}, 3 \mathrm{H}, J=7 \mathrm{~Hz}), 1.60(\mathrm{~m}, 9 \mathrm{H}), 2.04(\mathrm{~s}, 3 \mathrm{H}), 3.71(\mathrm{~m}, 2 \mathrm{H}), 3.79$ (dt, $1 \mathrm{H}, J=3.5,8.5 \mathrm{~Hz})$, $5.65(\mathrm{dd}, 1 \mathrm{H}, J=3,8.5 \mathrm{~Hz}) ;{ }^{13} \mathrm{C}$ NMR $\left(125 \mathrm{MHz}\right.$, acetone- $\left.\mathrm{d}_{6}\right) \mathrm{C}-5.8,11.8,20.9,25.3,25.6,28.6,30.1,35.7$, 59.6, 75.4, 94.9, 168.9; IR (neat) 2928, 2856, 1753, 1471, 1361, 1231, 1195, 1102, 1032, 1005, 954, 833, $774,661 \mathrm{~cm}^{-1}$; MS calculated for $\mathrm{C}_{16} \mathrm{H}_{32} \mathrm{O}_{4} \mathrm{Si}$ (M-59) 257, found 257.1 (M-59).
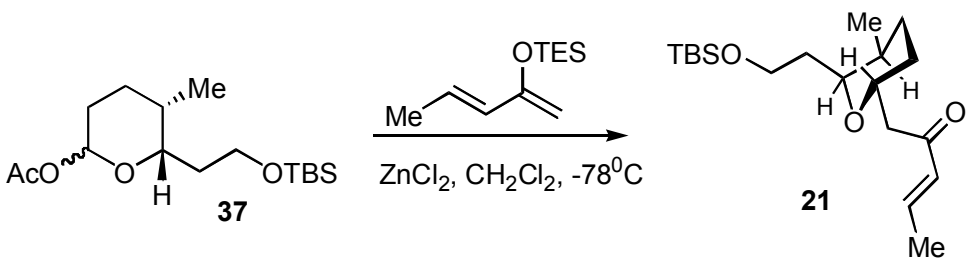

Enone 21. A solution of lactol acetate $37(1.33 \mathrm{~g}, 4.2 \mathrm{mmol})$ in $100 \mathrm{ml}$ of $\mathrm{CH}_{2} \mathrm{Cl}_{2}$ was cooled down to -78 ${ }^{\circ} \mathrm{C}$ and treated with silyl enol ether $(0.716 \mathrm{~g}, 4.2 \mathrm{mmol})$ followed by $\mathrm{ZnCl}_{2}\left(1 \mathrm{M}\right.$ in $\mathrm{Et}_{2} \mathrm{O}, 4.62 \mathrm{ml}, 4.62$ $\mathrm{mmol}$ ). The reaction mixture was allowed to warm to room temperature, stirred for $1.5 \mathrm{~h}$. and quenched with $\mathrm{NaHCO}_{3}$ (saturated solution, $40 \mathrm{ml}$ ), extracted with $\mathrm{CH}_{2} \mathrm{Cl}_{2}\left(40 \mathrm{ml}\right.$ ), dried with $\mathrm{Na}_{2} \mathrm{SO}_{4}$ and filtered. Filtrate was concentrated in vacuo to give enone $21(1.84 \mathrm{~g})$, which was used for the next step without any purification as purification by flash chromatography on silica gel caused significant loss of material; ${ }^{1} \mathrm{H}$ NMR $\left(500 \mathrm{MHz}, \mathrm{CDCl}_{3}\right) \square 0.06(\mathrm{~s}, 6 \mathrm{H}), 0.81(\mathrm{~d}, 3 \mathrm{H}, J=7 \mathrm{~Hz}), 0.89(\mathrm{~s}, 9 \mathrm{H}), 1.39(\mathrm{~m}, 3 \mathrm{H}), 1.59(\mathrm{~m}, 1 \mathrm{H})$, $1.70(\mathrm{dq}, 1 \mathrm{H}, J=3.1,12.8 \mathrm{~Hz}), 1.91(\mathrm{dd}, 3 \mathrm{H}, J=1.6,6.9 \mathrm{~Hz}), 2.47(\mathrm{dd}, 1 \mathrm{H}, J=5,15 \mathrm{~Hz}), 2.81(\mathrm{dd}, 1 \mathrm{H}, J=$ $8,15 \mathrm{~Hz}), 3.64(\mathrm{~m}, 1 \mathrm{H}), 3.72(\mathrm{~m}, 1 \mathrm{H}), 3.88(\mathrm{dt}, 1 \mathrm{H}, J=4,12 \mathrm{~Hz}), 4.02(\mathrm{~m}, 1 \mathrm{H}), 6.16(\mathrm{dd}, 1 \mathrm{H}, J=2,15$ $\mathrm{Hz}), 6.86(\mathrm{dq}, 1 \mathrm{H}, J=7,16 \mathrm{~Hz}) ;{ }^{13} \mathrm{C}$ NMR $\left(125 \mathrm{MHz}, \mathrm{CDCl}_{3}\right) \square$ 198.4, 142.8, 132.5, 73.7, 65.6, 59.8, 45.9, 32.9, 31.1, 27.9, 26.7, 25.9, 18.2, 18.1, 16.9. -5.3; MS calculated for $\mathrm{C}_{19} \mathrm{H}_{36} \mathrm{O}_{3} \mathrm{Si}(\mathrm{M}+)$ 340.57, found 341.2 $(\mathrm{M}+)$.

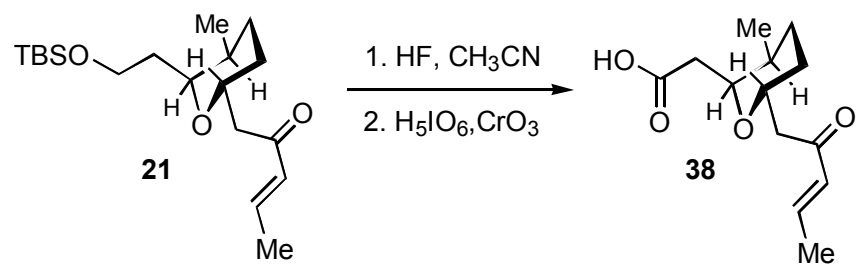

Acid 38. Enone 21 (1.84 g, $5.4 \mathrm{mmol}$ ) was dissolved in $100 \mathrm{ml}$ of $\mathrm{CH}_{3} \mathrm{CN}$ and treated with $\mathrm{HF}$ (49\%, $0.192 \mathrm{ml}, 5.5 \mathrm{mmol}$ ) at $0{ }^{\circ} \mathrm{C}$. The reaction mixture was stirred for 10 min and quenched with $\mathrm{NaHCO}_{3}$ (saturated solution, $30 \mathrm{ml})$. Organic layer was concentrated and treated with $\mathrm{Et}_{2} \mathrm{O}(100 \mathrm{ml})$ followed by $\mathrm{H}_{2} \mathrm{O}(20 \mathrm{ml})$. Aqueous phase was extracted with $\mathrm{Et}_{2} \mathrm{O}(50 \mathrm{ml})$. Combined organic extracts were washed with $\mathrm{NaHCO}_{3}$ (saturated solution, $20 \mathrm{ml}$ ), dried over $\mathrm{Na}_{2} \mathrm{SO}_{4}$, filtered and concentrated in vacuo to give the corresponding desilylated alcohol $(0.848 \mathrm{~g})$, which was used for the oxidation step without purification.

A stock solution of of $\mathrm{H}_{5} \mathrm{IO}_{6} / \mathrm{CrO}_{3}$ was prepared by dissolving $\mathrm{H}_{5} \mathrm{IO}_{6}(11.4 \mathrm{~g}, 50 \mathrm{mmol})$ and $\mathrm{CrO}_{3}(23 \mathrm{mg}$, $1.2 \% \mathrm{~mol})$ in wet $\mathrm{MeCN}(0.75 \mathrm{v} \%$ water) to a volume of $114 \mathrm{ml}$. Solution of alcohol prepared as described above $\left(418 \mathrm{mg}\right.$ ) in wet $\mathrm{CH}_{3} \mathrm{CN}(10 \mathrm{ml})$ was treated drop wise with $\mathrm{H}_{5} \mathrm{IO}_{6} / \mathrm{CrO}_{3}$ (stock solution, $10 \mathrm{ml}$ ) during $30 \mathrm{~min}$ while maintaining the reaction temperature at $0{ }^{\circ} \mathrm{C}$. After complete addition of the oxidant, the reaction mixture was stirred for $30 \mathrm{~min}$ at $0{ }^{\circ} \mathrm{C}$ and quenched with $\mathrm{Na}_{2} \mathrm{HPO}_{4}(1.8 \mathrm{~g}$ in $30 \mathrm{ml}$ of $\mathrm{H}_{2} \mathrm{O}$ ). Toluene $(35 \mathrm{ml})$ was added and the reaction mixture was stirred for $40 \mathrm{~min}$. Layers were separated. The aqueous phase was extracted with toluene $(30 \mathrm{ml})$. Remaining aqueous layer was treated with $\mathrm{HCl}$ $(37 \%, 1 \mathrm{ml})$ and extracted with toluene $(30 \mathrm{ml})$. Combined toluene extracts were washed with brine $(5 \mathrm{ml})$, 
water $(5 \mathrm{ml})$, dried with $\mathrm{Na}_{2} \mathrm{SO}_{4}$, filtered and concentrated in vacuo. The crude product was purified by flash chromatography on silica gel (elution with $\left.\mathrm{CH}_{2} \mathrm{Cl}_{2}: \mathrm{MeOH}=25: 1\right)$ to give acid $\mathbf{3 8}(184.5 \mathrm{mg}, 37 \%$ yield for 3 steps); ${ }^{1} \mathrm{H}$ NMR $\left(500 \mathrm{MHz}, \mathrm{C}_{6} \mathrm{D}_{6}\right) \square 0.43(\mathrm{~d}, 3 \mathrm{H}, J=6.9 \mathrm{~Hz}), 0.93(\mathrm{~m}, 2 \mathrm{H}), 1.11(\mathrm{~m}, 1 \mathrm{H}), 1.20$ $(\mathrm{dq}, 1 \mathrm{H}, J=4,13 \mathrm{~Hz}), 1.41(\mathrm{dd}, 3 \mathrm{H}, J=1.7,6.8 \mathrm{~Hz}), 1.55(\mathrm{dq}, 1 \mathrm{H}, J=3.5,13 \mathrm{~Hz}), 1.61(\mathrm{~m}, 1 \mathrm{H}), 2.10(\mathrm{dd}$, $1 \mathrm{H}, J=4,15 \mathrm{~Hz}), 2.34(\mathrm{dd}, 1 \mathrm{H}, J=6,15 \mathrm{~Hz}), 2.59(\mathrm{dd}, 1 \mathrm{H}, J=11,15 \mathrm{~Hz}), 2.77(\mathrm{dd}, 1 \mathrm{H}, J=7,16 \mathrm{~Hz})$, $4.12(\mathrm{~m}, 1 \mathrm{H}), 4.31(\mathrm{dt}, 1 \mathrm{H}, J=5,10 \mathrm{~Hz}), 6.01(\mathrm{dd}, 1 \mathrm{H}, J=1,15 \mathrm{~Hz}), 6.60(\mathrm{dq}, 1 \mathrm{H}, J=7,16 \mathrm{~Hz}), 10.29$ (br. s, 1H); ${ }^{13} \mathrm{C}$ NMR (125 MHz, $\left.\mathrm{C}_{6} \mathrm{D}_{6}\right) \square$ 197.6, 177.9, 143.0, 133.2, 74.6, 67.4, 46.5, 33.5, 33.4, 31.1, 27.1, 18.4, 17.0; MS calculated for $\mathrm{C}_{13} \mathrm{H}_{20} \mathrm{O}_{4}\left(\mathrm{M}+\mathrm{Na}^{23}\right) 262.3$, found $263.0\left(\mathrm{M}+\mathrm{Na}^{23}\right)$.

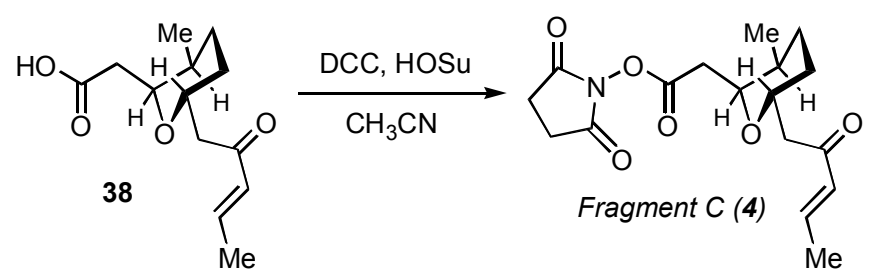

Ester 4. A solution of acid $38(19.5 \mathrm{mg}, 0.081 \mathrm{mmol})$ in $\mathrm{CH}_{3} \mathrm{CN}(1 \mathrm{ml})$ was treated with $\mathrm{N}$ hydroxysuccinimide $(9.5 \mathrm{mg}, 0.089 \mathrm{mmol})$ and dicyclohexylcarbodiimide $(17 \mathrm{mg}, 0.081 \mathrm{mmol})$. The reaction mixture was stirred for $1.5 \mathrm{~h}$ at room temperature. White precipitate was filtered off and filtrate was concentrated in vacuo. The crude product was purified by flash chromatography on silica gel (elution with $\left.\mathrm{CH}_{2} \mathrm{Cl}_{2}: \mathrm{MeOH}=20: 1\right)$ to give ester $4(27 \mathrm{mg}, 98 \%$ yield $)$; []$\left.^{25}\right]_{\mathrm{D}}-31.3^{\circ},\left(c 4.1, \mathrm{CH}_{3} \mathrm{CN}\right) ;{ }^{1} \mathrm{H} \mathrm{NMR}$ $\left(500 \mathrm{MHz}, \mathrm{CDCl}_{3}\right) \square 0.86(\mathrm{~d}, 3 \mathrm{H}, J=7 \mathrm{~Hz}), 1.34(\mathrm{~m}, 3 \mathrm{H}), 1.67(\mathrm{~m}, 3 \mathrm{H}), 1.82(\mathrm{~m}, 1 \mathrm{H}), 1.89(\mathrm{dd}, 3 \mathrm{H}, J=$ 1.4, 6.9Hz), $2.10(\mathrm{~m}, 1 \mathrm{H}), 2.63(\mathrm{dd}, 1 \mathrm{H}, J=8,16 \mathrm{~Hz}), 2.69$ (dd, $1 \mathrm{H}, J=5,15 \mathrm{~Hz}), 2.82$ (br.s, $4 \mathrm{H}), 2.96$ $(\mathrm{dd}, 1 \mathrm{H}, J=5,15 \mathrm{~Hz}), 3.03(\mathrm{dd}, 1 \mathrm{H}, J=10,15 \mathrm{~Hz}), 4.10(\mathrm{~m}, 1 \mathrm{H}), 4.37$ (quin., $1 \mathrm{H}, J=5 \mathrm{~Hz}), 6.11(\mathrm{dd}, 1 \mathrm{H}$, $J=2,16 \mathrm{~Hz}), 6.86(\mathrm{dq}, 1 \mathrm{H}, J=7,16 \mathrm{~Hz}) ;{ }^{13} \mathrm{C} \mathrm{NMR}\left(125 \mathrm{MHz}, \mathrm{CDCl}_{3}\right) \square$ 198.6, 169.1, 167.3, 143.5, 132.7, 73.9, 67.2, 45.5, 32.7, 30.6, 30.1, 26.5, 25.8, 18.5, 16.7; IR $\left(\mathrm{CH}_{3} \mathrm{CN}\right)$ 2944, 2292, 2252, 1742, 1443, $1374,1038,917 \mathrm{~cm}^{-1}$; MS calculated for $\mathrm{C}_{17} \mathrm{H}_{23} \mathrm{NO}_{6}\left(\mathrm{M}+\mathrm{Na}^{23}\right) 360.14$, found $360\left(\mathrm{M}+\mathrm{Na}^{23}\right)$.

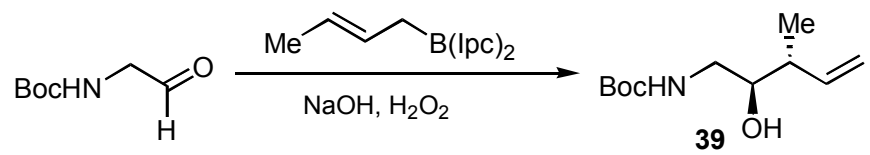

Alcohol 39. To a stirred suspension of $t$-BuOK $(2.8 \mathrm{~g}, 24.9 \mathrm{mmol})$ in THF $(60 \mathrm{~mL})$ were added trans-2butene $(7.68 \mathrm{~g}, 137 \mathrm{mmol})$ and $n$-BuLi $(1.6 \mathrm{M}$ in hexanes, $15.64 \mathrm{~mL}, 25 \mathrm{mmol})$ at $-78{ }^{\circ} \mathrm{C}$. The solution was warmed up to $-45^{\circ} \mathrm{C}$, stirred for $10 \mathrm{~min}$, cooled back to $-78{ }^{\circ} \mathrm{C}$. Solution of $(+)-(\mathrm{Ipc}){ }_{2} \mathrm{BOMe}(7.87 \mathrm{~g}$, $24.9 \mathrm{mmol})$ in $23 \mathrm{ml}$ of THF was added drop wise at $-78{ }^{\circ} \mathrm{C}$. After $30 \mathrm{~min}, \mathrm{BF}_{3} \bullet \mathrm{OEt}_{2}(3.17 \mathrm{ml}, 24.9$ mmol) was added, followed by Boc-protected aldehyde ${ }^{4}(3.61 \mathrm{~g}, 22.6 \mathrm{mmol})$ dissolved in THF $(10 \mathrm{ml})$. The resulting mixture was stirred for $3 \mathrm{~h}$ at $-78{ }^{\circ} \mathrm{C}$, quenched with $\mathrm{NaOH}(3 \mathrm{M}, 24 \mathrm{~mL})$ and $\mathrm{H}_{2} \mathrm{O}_{2}(30 \mathrm{wt} \%$, $24 \mathrm{~mL})$, and stirred overnight. Aqueous phase was extracted with EtOAc $(100 \mathrm{ml})$. Combined organic extracts were dried with $\mathrm{MgSO}_{4}$, filtered, and concentrated in vacuo. The crude product was purified by flash chromatography on silica gel (elution with EtOAc:hexane $=1: 9)$ to give alcohol $39(3.11 \mathrm{~g}, 64 \%$ yield, 93\% ee - determined by Mosher ester analysis); $[\square]^{25}-2.63^{\circ},\left(c 5.8, \mathrm{CHCl}_{3}\right) ;{ }^{1} \mathrm{H} \mathrm{NMR}(500 \mathrm{MHz}$, $\left.\mathrm{CDCl}_{3}\right) \square 1.05(\mathrm{~d}, 3 \mathrm{H}, J=6.8 \mathrm{~Hz}$ ), $1.45(\mathrm{~s}, 9 \mathrm{H}), 2.23$ (quin., $1 \mathrm{H}, J=7 \mathrm{~Hz}$ ), 2.35 (br.s, $1 \mathrm{H}), 3.07$ (ddd, $1 \mathrm{H}$, $J=6,8,14 \mathrm{~Hz}$ ), 3.35 (br.m, 1H), 3.49 (br.m, 1H), 4.95 (br.s, 1H), 5.13 (m, 2H), 5.76 (m, 1H); ${ }^{13} \mathrm{C}$ NMR $\left(125 \mathrm{MHz}, \mathrm{CDCl}_{3}\right) \square$ 156.7, 139.9, 116.4, 79.5, 74.2, 44.3, 42.3, 28.4, 18.1; IR (neat) 3357, 2975, 1685, $1510,1365,1249,1166,1093,1041,999,914,885,778,736 \mathrm{~cm}^{-1}$; MS calculated for $\mathrm{C}_{11} \mathrm{H}_{21} \mathrm{NO}_{3}\left(\mathrm{M}+\mathrm{Na}^{23}\right)$ 238.28 , found $238.1\left(\mathrm{M}+\mathrm{Na}^{23}\right)$. 


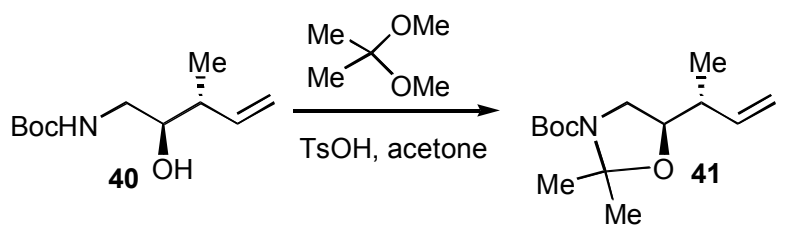

Acetonide 41. A solution of alcohol 40 (1.02 g, $4.74 \mathrm{mmol})$ in acetone (40 ml) was treated with 2,2dimethoxy propane $(10 \mathrm{ml})$ and $\mathrm{TsOH}$ monohydrate $(85 \mathrm{mg}, 0.4 \mathrm{mmol})$. The reaction mixture was stirred for 5 min. and quenched with $\mathrm{NaHCO}_{3}$ (saturated solution, $40 \mathrm{ml}$ ). Aqueous layer was extracted with additional amount of $\mathrm{CH}_{2} \mathrm{Cl}_{2}(50 \mathrm{ml})$. Combined organic layers were dried over $\mathrm{MgSO}_{4}$, filtered and concentrated in vacuo. The crude product was purified by flash chromatography on silica gel (elution with EtOAc:hexane = 1:9) to give acetonide $41(1.09 \mathrm{~g}, 90 \%$ yield $)$; [ $]^{25}{ }_{\mathrm{D}}-19.08^{\circ},\left(c 2.7, \mathrm{CHCl}_{3}\right) ;{ }^{1} \mathrm{H} \mathrm{NMR}$ $\left(500 \mathrm{MHz}, \mathrm{CDCl}_{3}, 340 \mathrm{~K}\right) \square 1.00(\mathrm{~d}, 3 \mathrm{H}, J=6.9 \mathrm{~Hz}), 1.43(\mathrm{~s}, 9 \mathrm{H}), 1.44(\mathrm{~s}, 3 \mathrm{H}), 1.50(\mathrm{~s}, 3 \mathrm{H}), 2.34$ (quintet, $1 \mathrm{H}, J=6.2 \mathrm{~Hz}), 3.11(\mathrm{t}, 1 \mathrm{H}, J=9.6 \mathrm{~Hz}), 3.54$ (br.s, $1 \mathrm{H}), 3.89(\mathrm{dt}, 1 \mathrm{H}, J=6.2,9.3 \mathrm{~Hz}), 5.1(\mathrm{~m}, 2 \mathrm{H}), 5.80$ $(\mathrm{ddd}, 1 \mathrm{H}, J=7,10.4,17.0 \mathrm{~Hz}) ;{ }^{13} \mathrm{C} \mathrm{NMR}\left(125 \mathrm{MHz}, \mathrm{CDCl}_{3}\right.$, room temperature) $\square$ 152.4, 151.9, 139.7, 115.2, 93.5, 93.1, 80.0, 79.3, 76.9, 48.8, 48.7, 40.4, 28.4, 27.1, 26.0, 25.2, 24.2, 15.4; IR (neat) 2976, 2934, $1696,1455,1389,1364,1253,1175,1148,1093,1050,914,874,769,733,679 \mathrm{~cm}^{-1}$; MS calculated for $\mathrm{C}_{14} \mathrm{H}_{25} \mathrm{NO}_{3}(\mathrm{M}+1) 256.18$, found $256(\mathrm{M}+1)$.

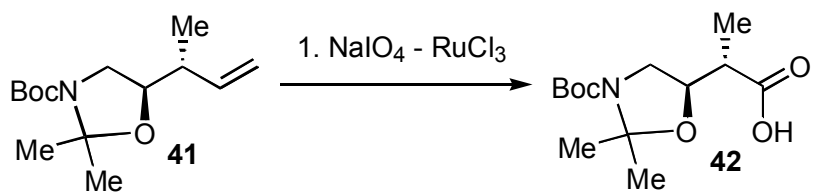

Acid 42. A solution of acetonide $41(3.96 \mathrm{~g}, 15.5 \mathrm{mmol})$ in $\mathrm{CCl}_{4}(30 \mathrm{ml})$ was treated with $\mathrm{CH}_{3} \mathrm{CN}(30 \mathrm{ml})$ and $\mathrm{H}_{2} \mathrm{O}(46 \mathrm{ml})$. The resulting mixture was vigorously stirred and treated with $\mathrm{NaIO}_{4}(13.57 \mathrm{~g}, 63.44$ mmol followed by $\mathrm{RuCl}_{3}(0.164 \mathrm{~g}, 0.79 \mathrm{mmol})$. Stirring was continued for $12 \mathrm{~h}$ at room temperature. The reaction mixture was diluted with $\mathrm{CH}_{2} \mathrm{Cl}_{2}(50 \mathrm{ml})$ and $\mathrm{H}_{2} \mathrm{O}(50 \mathrm{ml})$ and the resulting green cocktail was filtered through the fritted filter. Organic layers were separated, and the aqueous phase was extracted with $\mathrm{CH}_{2} \mathrm{Cl}_{2}(3 \square 50 \mathrm{ml})$. Combined organic layers were dried with $\mathrm{MgSO}_{4}$, filtered and evaporated. The crude product was purified by flash chromatography on silica gel (elution with EtOAc: $\mathrm{CH}_{3} \mathrm{COOH}=100: 1$ ) to give acid 42 (2.65 g, 62\% yield, yellow solid); [ $]_{\mathrm{D}}^{25}-15.2^{\circ},\left(\mathrm{c} 2.07, \mathrm{CHCl}_{3}\right) ;{ }^{1} \mathrm{H} \mathrm{NMR}\left(500 \mathrm{MHz}, \mathrm{CDCl}_{3}\right.$, $340 \mathrm{~K}) \square 1.22(\mathrm{~d}, 3 \mathrm{H}, J=7.2 \mathrm{~Hz}), 1.47(\mathrm{~s}, 9 \mathrm{H}), 1.50(\mathrm{~s}, 3 \mathrm{H}), 1.57(\mathrm{~s}, 3 \mathrm{H}), 2.70$ (quintet, 1H, $J=7.0 \mathrm{~Hz}$ ), $3.22(\mathrm{t}, 1 \mathrm{H}, J=10 \mathrm{~Hz}), 3.71(\mathrm{dd}, 1 \mathrm{H}, J=6,10 \mathrm{~Hz}), 4.25(\mathrm{~m}, 1 \mathrm{H}) ;{ }^{13} \mathrm{C} \mathrm{NMR}\left(125 \mathrm{MHz}, \mathrm{CDCl}_{3}, \mathrm{room}\right.$ temperature) $\square 178.9,153.0 .152 .5,81.0,79.5,74.2,48.3,48.1,43.0,42.7,28.3,27.0,25.9,25.1,24.2$, $12.7,12.5$.

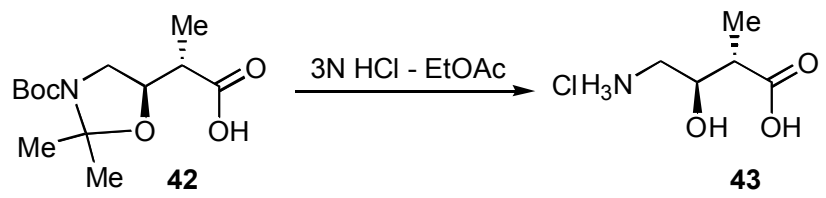

Amino Acid Hydrochloride 43. A solution of acid 42 (2.65 g, $9.7 \mathrm{mmol})$ in EtOAc (50 ml) was treated with $\mathrm{HCl}(3 \mathrm{~N}, 50 \mathrm{ml})$. The reaction mixture was heated to $36^{\circ} \mathrm{C}$, stirred until disappearance of the starting material as monitored by TLC, diluted with $\mathrm{H}_{2} \mathrm{O}(50 \mathrm{ml})$ and EtOAc $(50 \mathrm{ml})$. Organic layer was separated, and the aqueous phase was washed with EtOAc $(50 \mathrm{ml})$. Aqueous layer was collected and concentrated in vacuo to give amino acid hydrochloride $43(1.63 \mathrm{~g}, 100 \% \text { yield, brown solid). [ }]^{25}{ }_{\mathrm{D}}-7.05^{\circ},\left(c 1.4, \mathrm{H}_{2} \mathrm{O}\right)$; ${ }^{1} \mathrm{H}$ NMR $\left(500 \mathrm{MHz}, \mathrm{D}_{2} \mathrm{O}\right) \square 1.08$ (d, 3H, $J=7.1 \mathrm{~Hz}$ ), 2.57 (quint., $1 \mathrm{H}, J=7.1 \mathrm{~Hz}$ ), 2.93 (dd, 1H, $J=10.4$, $12.9 \mathrm{~Hz}), 3.17(\mathrm{dd}, 1 \mathrm{H}, J=2.4,13.1 \mathrm{~Hz}), 3.90(\mathrm{ddd}, 1 \mathrm{H}, J=2.8,7.2,10.2 \mathrm{~Hz}), 4.68(\mathrm{br} . \mathrm{s}, 3 \mathrm{H})$; ${ }^{13} \mathrm{C} \mathrm{NMR}$ $\left(125 \mathrm{MHz}, \mathrm{D}_{2} \mathrm{O}\right) \square 178.3,69.5,44.4,42.6,13.0$; MS calculated for $\mathrm{C}_{5} \mathrm{H}_{12} \mathrm{NO}_{3}(\mathrm{M}+)$ 134.08, found 134.1. 


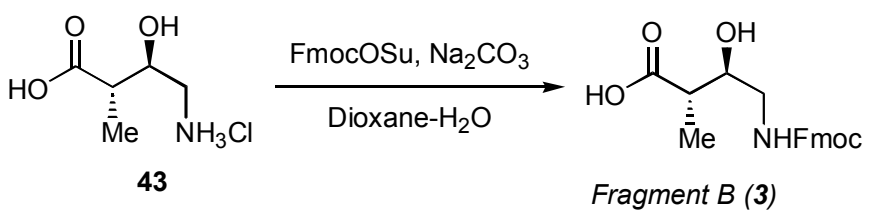

Acid 3. A solution of hydrochloride $43(113 \mathrm{mg}, 0.666 \mathrm{mmol})$ in $\mathrm{H}_{2} \mathrm{O}(1.5 \mathrm{ml})$ and 1,4-dioxane (1.5 $\left.\mathrm{ml}\right)$ was treated with FmocOSu (224 mg, $0.666 \mathrm{mmol})$ and $\mathrm{Na}_{2} \mathrm{CO}_{3}$ (282 $\left.\mathrm{mg}, 2.664 \mathrm{mmol}\right)$. The reaction mixture was stirred for $1 \mathrm{~h}$ at room temperature, poured into $\mathrm{H}_{2} \mathrm{O}(5 \mathrm{ml})$ and washed with EtOAc $(3 \square 5 \mathrm{ml})$.

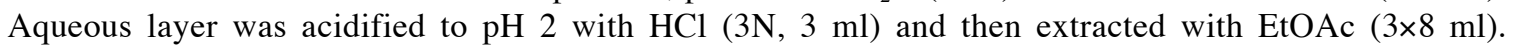
Combined organic layers were dried with anhydrous $\mathrm{Na}_{2} \mathrm{SO}_{4}$, filtered and concentrated in vacuo. The crude product was purified by flash chromatography on silica gel (elution with $\mathrm{CH}_{2} \mathrm{Cl}_{2}: \mathrm{MeOH} \mathrm{CH}_{3} \mathrm{COOH}=$ 92:5:1) to give N-protected amino acid $3(160 \mathrm{mg}, 71 \% \text { yield); [ }]^{25}{ }_{\mathrm{D}}+2.44^{\circ},\left(c 1.48, \mathrm{CH}_{3} \mathrm{CN}\right) ;{ }^{1} \mathrm{H} \mathrm{NMR}$ $\left(500 \mathrm{MHz}, \mathrm{CHCl}_{3}\right) \square 1.22(\mathrm{~d}, 3 \mathrm{H}, J=7 \mathrm{~Hz}), 2.61$ (quint., $\left.1 \mathrm{H}, J=7.5 \mathrm{~Hz}\right), 3.24(\mathrm{~m}, 1 \mathrm{H}), 3.38(\mathrm{~d}, 1 \mathrm{H}, J=$ $5.5 \mathrm{~Hz}), 3.46(\mathrm{~m}, 1 \mathrm{H}), 4.20(\mathrm{t}, 1 \mathrm{H}, J=6.8 \mathrm{~Hz}), 4.41(\mathrm{~d}, 2 \mathrm{H}, J=7 \mathrm{~Hz}), 5.32$ (br.t, 1H), $7.31(\mathrm{t}, 2 \mathrm{H}, J=7.0$ $\mathrm{Hz}), 7.39(\mathrm{t}, 2 \mathrm{H}, J=7.5 \mathrm{~Hz}), 7.59(\mathrm{~d}, 2 \mathrm{H}, J=7.5 \mathrm{~Hz}), 7.77(\mathrm{~d}, 2 \mathrm{H}, J=7.5 \mathrm{~Hz}) ;{ }^{13} \mathrm{C} \mathrm{NMR}(125 \mathrm{MHz}$, $\left.\mathrm{CD}_{3} \mathrm{OD}\right) \square 143.8,141.9,128.0,127.4,125.4,120.1,72.3,66.7,44.3,44.1,43.7,12.6$; IR (neat) 1700, 1539, $1449,1260,750,740 \mathrm{~cm}^{-1}$. MS calculated for $\mathrm{C}_{20} \mathrm{H}_{21} \mathrm{NO}_{5}(\mathrm{M}+1) 356.14$, found 356.10 .
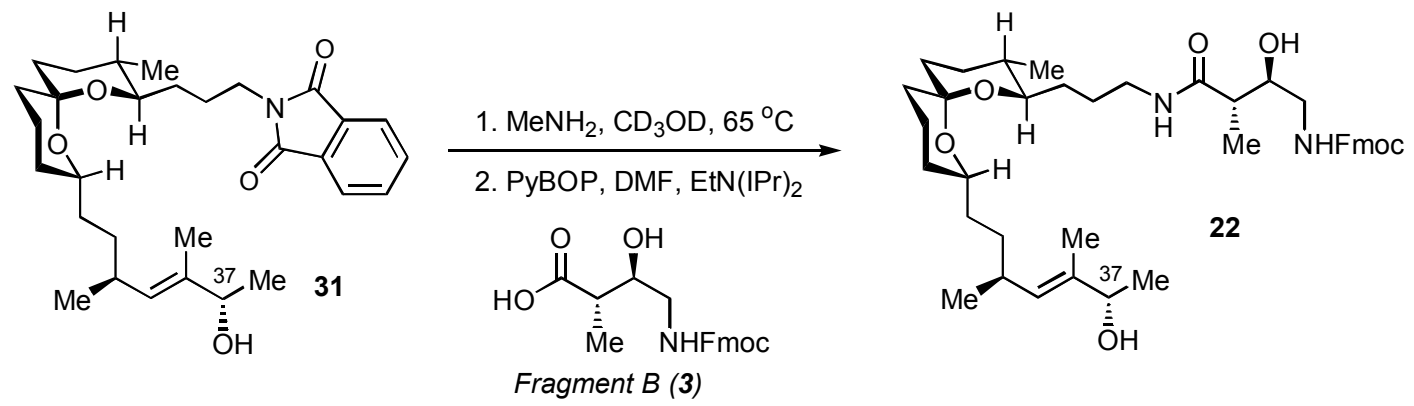

(37S)-Amide 22. A solution of (37S)-alcohol $31(18 \mathrm{mg}, 0.036 \mathrm{mmol})$ in $\mathrm{CD}_{3} \mathrm{OD}(0.8 \mathrm{~mL})$ was treated with $\mathrm{MeNH}_{2}(40 \%$ solution in water, $60 \square \mathrm{L}, 0.72 \mathrm{mmol})$. The reaction mixture was kept for $3 \mathrm{~h}$ at $65^{\circ} \mathrm{C}$. Solvent was removed in vacuo. Excess of $\mathrm{MeNH}_{2}$ was removed under high vacuum to furnish deprotected amino alcohol (13 mg, quantitative), which was used directly in next step without further purification.

Acid 3 (8.52 mg, $0.024 \mathrm{mmol})$ was dissolved in DMF $(0.5 \mathrm{ml})$ and treated via syringe with a solution of the amino alcohol described above $(0.024 \mathrm{mmol})$, obtained after phtalimide deprotection, in DMF $(0.5 \mathrm{ml})$. The reaction mixture was treated with PyBOP $(12.5 \mathrm{mg}, 0.024 \mathrm{mmol})$ and $\mathrm{EtN}(\mathrm{iPr})_{2}(8.4 \mathrm{ul}, 0.048 \mathrm{mmol})$ and stirred at room temperature for $2 \mathrm{~h}$. DMF was removed by bulb-to-bulb distillation at $1 \mathrm{~mm} \mathrm{Hg}$, at $35^{\circ} \mathrm{C}$. The crude product was purified by flash chromatography on silica gel (elution with EtOAc $-\mathrm{Hex}-4: 1->$ $3: 1)$ to give amide $22(14.4 \mathrm{mg}, 85 \%$ yield $)$; $[\square]_{\mathrm{D}}^{25}+15.86^{\circ},\left(c 1.4, \mathrm{CH}_{3} \mathrm{CN}\right) ;{ }^{1} \mathrm{H}$ NMR $\left(500 \mathrm{MHz}, \mathrm{CD}_{3} \mathrm{CN}\right)$ $\square 0.69(\mathrm{~d}, 3 \mathrm{H}, J=6.53 \mathrm{~Hz}), 0.83(\mathrm{~d}, 3 \mathrm{H}, J=6.6 \mathrm{~Hz}), 1.04(\mathrm{~d}, 5 \mathrm{H}, J=6.5 \mathrm{~Hz}), 1.21(\mathrm{~m}, 10 \mathrm{H}), 1.33(\mathrm{~m}, 9 \mathrm{H})$, 1.46 (d, 3H, J=1.2 Hz), 1.56 (br.m, 1H), 1.71 (br.m, 2H), 2.13 (br.s, 8H), 2.97 (m, 1H), 3.07 (m, 3H), 3.13 $(\mathrm{m}, 1 \mathrm{H}), 3.37(\mathrm{~m}, 1 \mathrm{H}), 3.46$ (br.s, $1 \mathrm{H}), 3.96(\mathrm{q}, 1 \mathrm{H}, J=6.75 \mathrm{~Hz}), 4.13(\mathrm{t}, 1 \mathrm{H}, J=6.8 \mathrm{~Hz}), 4.25(\mathrm{dd}, 2 \mathrm{H}, J=$ 2.9, 7.2 Hz), 4.99 (d, 1H, $J=9.5 \mathrm{~Hz}$ ), 5.72 (br.t, 1H), 6.58 (br.t, 1H), 7.24 (t, 2H, J = 7.3 Hz), 7.32 (t, $2 \mathrm{H}, J$ $=7.4 \mathrm{~Hz}), 7.57(\mathrm{~d}, 2 \mathrm{H}, J=7.4 \mathrm{~Hz}), 7.74(\mathrm{~d}, 2 \mathrm{H}, J=7.6 \mathrm{~Hz}) ;{ }^{13} \mathrm{C} \mathrm{NMR}\left(125 \mathrm{MHz}, \mathrm{CD}_{3} \mathrm{CN}\right) \square$ 175.2, 157.0, 144.1, 141.1, 137.7, 130.2, 127.6, 127.1, 125.1, 119.9, 95.1, 74.0, 72.8, 72.3, 68.6, 66.08, 54.3, 47.1, 44.9, $42.5,39.0,35.9,35.2,34.8,33.9,33.4,33.3,31.5,31.2,30.2,27.7,25.6,21.6,20.5,19.0,17.3,14.7$; IR (film) $3639,3422,2931,1701,1635,1539,1450,1261,846,758 \mathrm{~cm}^{-1}$. MS peaks 721, 720, 686.9, 686.0, 509.7. Calculated for $\mathrm{C}_{42} \mathrm{H}_{60} \mathrm{~N}_{2} \mathrm{O}_{7}(\mathrm{M}+)$ 704.94.18. 

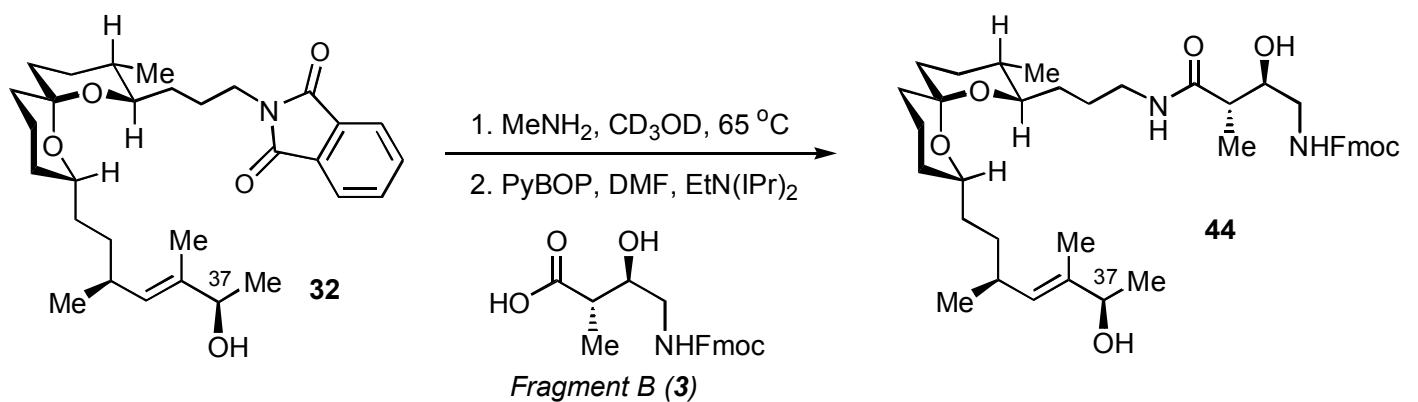

(37R)-amide 44. A solution of (37R)-alcohol $32(19 \mathrm{mg}, 0.037 \mathrm{mmol})$ in $\mathrm{CD}_{3} \mathrm{OD}(0.8 \mathrm{~mL})$ was treated with $\mathrm{MeNH}_{2}(40 \%$ solution in water, $60 \square \mathrm{L}, 0.72 \mathrm{mmol})$. The reaction mixture was kept for $3 \mathrm{~h}$ at $65^{\circ} \mathrm{C}$. Solvent was removed in vacuo. Excess of $\mathrm{MeNH}_{2}$ was removed under high vacuum to furnish the corresponding deprotected amino alcohol (14 mg, quantitative), which was used directly in next step without further purification.

Acid 3 (13 mg, $0.037 \mathrm{mmol})$ was dissolved in DMF $(0.5 \mathrm{ml})$ and treated via syringe with a solution of the $(37 R)$-amino alcohol described above $(0.037 \mathrm{mmol})$, obtained after phtalimide deprotection, in DMF $(0.5 \mathrm{ml})$. The reaction mixture was treated with PyBOP $(19 \mathrm{mg}, 0.036 \mathrm{mmol})$ and EtN(iPr) $2(6.3 \mathrm{ul}, 0.036$ $\mathrm{mmol}$ ) and stirred at room temperature for $2 \mathrm{~h}$. DMF was removed by bulb-to-bulb distillation at $1 \mathrm{~mm} \mathrm{Hg}$, at $35{ }^{\circ} \mathrm{C}$. The crude product was purified by flash chromatography on silica gel (elution with EtOAc $-\mathrm{Hex}$ $-4: 1->3: 1)$ to give amide (37R)-amide 44 (19 $\mathrm{mg}, 76 \%$ yield), which was used directly for the next steps.

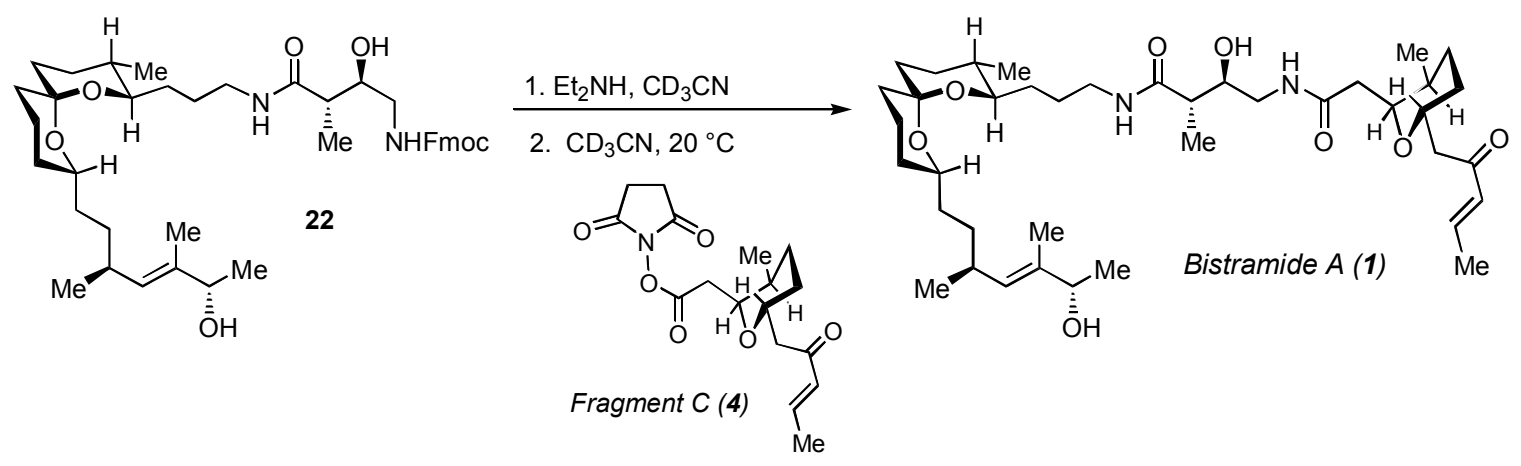

Bistramide A (1). A solution of (37S)-amide $22(9.3 \mathrm{mg}, 13.2 \square \mathrm{m})$ in DMF $(0.6 \mathrm{ml})$ was treated with $\mathrm{Et}_{2} \mathrm{NH}(0.2 \mathrm{ml})$. The reaction mixture was stirred for $50 \mathrm{~min}$ at room temperature; $\mathrm{Et}_{2} \mathrm{NH}$ was evaporated under reduced pressure and DMF was removed by bulb-to-bulb distillation at $1 \mathrm{~mm} \mathrm{Hg}$ at $35{ }^{\circ} \mathrm{C}$. The residue was treated with the solution of $4(6.45 \mathrm{mg}, 19 \square \mathrm{m})$ in DMF $(0.75 \mathrm{ml})$ and the resulting mixture was stirred at room temperature for $13 \mathrm{~h}$. DMF was removed by bulb-to-bulb distillation at $1 \mathrm{~mm} \mathrm{Hg}$ at 35 ${ }^{\circ} \mathrm{C}$, and the crude product was purified by flash chromatography on silica gel (elution with $\mathrm{CHCl}_{3}: \mathrm{MeOH}^{-}$ 97:3) to give Bistramide A (1) (6 mg, 65\% yield for 2 steps). The product was further purified via reverse phase HPLC (Microsorb $100 \mathrm{C} 18,250 \mathrm{~mm} \square 21.4 \mathrm{~mm}, \mathrm{MeOH}: \mathrm{H}_{2} \mathrm{O}=75: 15$, flow rate $21.6 \mathrm{ml} / \mathrm{min}, \square=254$ $\left.\mathrm{nm}, \mathrm{T}_{\mathrm{r}}=30.6 \mathrm{~min}\right) ;{ }^{1} \mathrm{H} \mathrm{NMR}\left(500 \mathrm{MHz} \mathrm{CDCl}_{3}\right) \square 0.80(\mathrm{~d}, 3 \mathrm{H}, J=6.5 \mathrm{~Hz}), 0.85(\mathrm{~d}, 3 \mathrm{H}, J=6.9 \mathrm{~Hz}), 0.94$ $(\mathrm{d}, 3 \mathrm{H}, J=6.6 \mathrm{~Hz}), 1.25(\mathrm{t}, 6 \mathrm{H}, J=8.1,6.5 \mathrm{~Hz}), 1.28-1.40(\mathrm{~m}, 8 \mathrm{H}), 1.40-1.45$ (m, 2H), 1.48-1.78 (br.m, 17H), 1.78-1.87 (br.m, 1H), 1.91 (dd, 3H, $J=1.4,6.7 \mathrm{~Hz}), 2.13$ (dd, 1H, $J=1.0,15 \mathrm{~Hz}), 2.31-2.39$ (m, 2H), $2.52(\mathrm{dd}, 1 \mathrm{H}, J=2,17 \mathrm{~Hz}), 2.75(\mathrm{dd}, 1 \mathrm{H}, J=15,12 \mathrm{~Hz}), 2.90(\mathrm{dd}, 1 \mathrm{H}, J=17,9 \mathrm{~Hz}), 3.14(\mathrm{dt}, 1 \mathrm{H}, J=$ $2,10 \mathrm{~Hz}), 3.22(\mathrm{dt}, 1 \mathrm{H}, J=13,6 \mathrm{~Hz}), 3.29(\mathrm{q}, 2 \mathrm{H}, J=6,13 \mathrm{~Hz}), 3.40-3.46(\mathrm{~m}, 1 \mathrm{H}), 3.49(\mathrm{dt}, 1 \mathrm{H}, J=6,14$ $\mathrm{Hz}), 3.71$ (quint., $1 \mathrm{H}), 4.05(\mathrm{dd}, 1 \mathrm{H}, J=5,11 \mathrm{~Hz}), 4.16-4.20(\mathrm{~m}, 2 \mathrm{H}), 4.61(\mathrm{~d}, 1 \mathrm{H}, J=5 \mathrm{~Hz}), 5.17(\mathrm{~d}, 1 \mathrm{H}, 9$ $\mathrm{Hz}), 6.11(\mathrm{dd}, 1 \mathrm{H}, J=1,15 \mathrm{~Hz}), 6.90(\mathrm{dq}, 1 \mathrm{H}, J=7,16 \mathrm{~Hz}), 6.94$ (br.t, $1 \mathrm{H}, J=5 \mathrm{~Hz}), 7.32$ (br.t, $1 \mathrm{H}, J=6$ $\mathrm{Hz}) ;{ }^{13} \mathrm{C}$ NMR $\left(125 \mathrm{MHz}, \mathrm{CDCl}_{3}\right) \square 199.1,175.3,173.7,144.7,137.3,132.3,131.5,95.6,74.9,74.4,73.9$, $73.4,69.2,64.8,45.4,44.8,43.5,39.6,36.2$, 35.6, 35.0, 34.2, 33.6, 33.5, 32.4, 32.0, 31.5, 30.9, 30.6, 28.0, 26.7, 26.0, 21.9, 21.1, 19.4, 18.6, 18.1, 17.3, 15.7, 11.9; MS calculated for $\mathrm{C}_{40} \mathrm{H}_{68} \mathrm{~N}_{2} \mathrm{O}_{8}\left(\mathrm{M}+\mathrm{Na}^{23}\right) 727.49$, 
found $727.3\left(\mathrm{M}+\mathrm{Na}^{23}\right) .{ }^{1} \mathrm{H}$ and ${ }^{13} \mathrm{C}$ NMR spectra of the synthetic sample of bistramide A were identical to those of the natural sample. Upon mixing synthetic bistramide A with the natural bistramide A, one set of signals was observed in the ${ }^{1} \mathrm{H}$ and ${ }^{13} \mathrm{C}$ spectra. For side-by-side comparison of the ${ }^{13} \mathrm{C}$ NMR data, see Table 1 on page S16. Reverse phase HPLC (Microsorb $100 \mathrm{C} 18,250 \mathrm{~mm} \square$ 4.6mm, MeOH: $\mathrm{H}_{2} \mathrm{O}=75: 15$, flow rate $1 \mathrm{ml} / \mathrm{min}, \square=254 \mathrm{~nm}$ ) of the mixture of synthetic and natural samples of natural products showed only one peak with the retention time of 30.6 min. Optical rotation of the synthetic sample of bistramide A was determined to be $[\square]_{\mathrm{D}}^{25}+6.4^{\circ},\left(c 0.30, \mathrm{CH}_{2} \mathrm{Cl}_{2}\right)$; While the reported optical rotation value for natural bistramide $\mathrm{A}$ is $+10^{\circ}\left(\mathrm{c} 0.5, \mathrm{CH}_{2} \mathrm{Cl}_{2}\right),{ }^{5}$ our measurement of the optical rotation of the natural sample of bistramide A was $[\square]_{\mathrm{D}}^{25}+8.6^{\circ},\left(c 0.69, \mathrm{CH}_{2} \mathrm{Cl}_{2}\right)$.

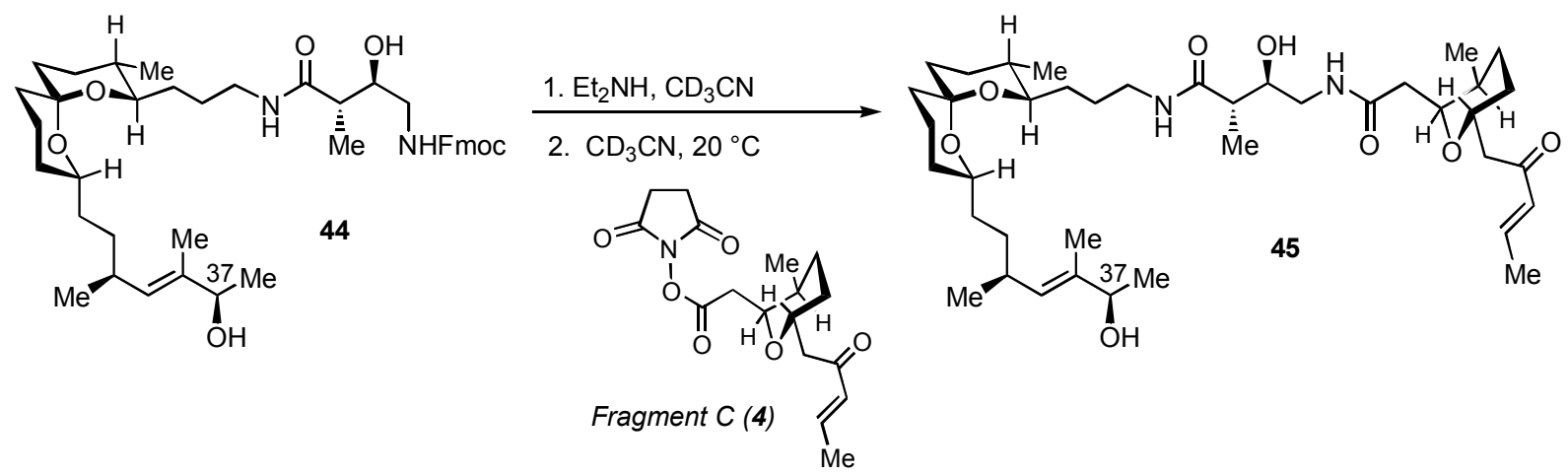

(37R)-Epimer of Bistramide A (45). A solution of (37R)-amide $44(10.8 \mathrm{mg}, 15.3 \square \mathrm{m})$ in DMF (0.7 ml) was treated with $\mathrm{Et}_{2} \mathrm{NH}(0.2 \mathrm{ml})$. The reaction mixture was stirred for $35 \mathrm{~min}$ at room temperature; $\mathrm{Et}_{2} \mathrm{NH}$ was evaporated under reduced pressure and DMF was removed by bulb-to-bulb distillation at $1 \mathrm{~mm} \mathrm{Hg}$ at $35^{\circ} \mathrm{C}$. The residue was treated with the solution of $4(13 \mathrm{mg}, 38 \square \mathrm{m})$ in DMF $(0.75 \mathrm{ml})$ and the resulting mixture was stirred at room temperature for $13 \mathrm{~h}$. DMF was removed by bulb-to-bulb distillation at $1 \mathrm{~mm}$ $\mathrm{Hg}$ at $35^{\circ} \mathrm{C}$, and the residue was purified by flash column chromatography (elution with $\mathrm{CHCl}_{3}-\mathrm{MeOH}-$ 97:3) to give (37R)-epimer of bistramide A 45 (7 mg, 64\% yield over 2 steps); [ []$^{25}{ }_{\mathrm{D}}+5.0^{\circ},(c 0.14$, $\left.\mathrm{CH}_{2} \mathrm{Cl}_{2}\right)^{1} \mathrm{H}$ NMR (500 MHz, $\left.\mathrm{CDCl}_{3}\right) \square 0.80(\mathrm{~d}, 3 \mathrm{H}, J=6.5 \mathrm{~Hz}), 0.86(\mathrm{~d}, 3 \mathrm{H}, J=6 . \mathrm{Hz}), 0.91(\mathrm{~d}, 3 \mathrm{H}, J=$ $6.6 \mathrm{~Hz}$ ), $1.10-1.20$ (br.m, $2 \mathrm{H}), 1.23$ (d, $3 \mathrm{H}, J=6.6 \mathrm{~Hz}), 1.26$ (d, $3 \mathrm{H}, J=7.3 \mathrm{~Hz}), 1.30-1.45(\mathrm{~m}, 4 \mathrm{H}), 1.40-$ 1.78 (br.s, 16H), 1.78-1.85 (br.m, $1 \mathrm{H}), 1.91$ (dd, $3 \mathrm{H}, J=1.5,6.8 \mathrm{~Hz}), 2.13$ (d, $1 \mathrm{H}, \mathrm{J}=15.3 \mathrm{~Hz}), 2.43-2.30$ (m, $2 \mathrm{H}), 2.52(\mathrm{dd}, 1 \mathrm{H}, J=2.8,17 \mathrm{~Hz}), 2.70-2.77(\mathrm{~m}, 1 \mathrm{H}), 2.89(\mathrm{dd}, 1 \mathrm{H}, J=17,8.9 \mathrm{~Hz}), 2.94(\mathrm{~d}, 1 \mathrm{H}, 4.8 \mathrm{~Hz})$, 3.15 (t, $1 \mathrm{H}, J=9.9 \mathrm{~Hz}), 3.20-3.38(\mathrm{~m}, 3 \mathrm{H}), 3.38-3.55(\mathrm{~m}, 2 \mathrm{H}), 3.70$ (br.s, $1 \mathrm{H}), 4.06(\mathrm{dd}, 1 \mathrm{H}, J=4.6$, $10.8 \mathrm{~Hz}$ ), 4.10-4.25 (m, 2H), 5.17 (d, $1 \mathrm{H}, J=9.2 \mathrm{~Hz}), 6.11$ (dd, $1 \mathrm{H}, J=15 \mathrm{~Hz}$ ), 6.88 (sept., $1 \mathrm{H}$ ), 6.96 (br.t, 1H), 7.31 (br.t, 1H); ${ }^{13} \mathrm{C}$ NMR (125 MHz, $\mathrm{CDCl}_{3}$ ) 199.1, 175.4, 173.6, 144.7, 137.4, 132.3, 131.6, 95.6, 74.9, 74.3, 74.0, 73.5, 69.4, 64.9, 45.4, 44.8, 43.4, 39.5, 36.2, 35.6, 35.1, 34.2, 33.7, 33.5, 32.4, 32.0, 31.4, $30.9,30.4,28.0,26.7,25.9,21.8,21.0,19.4,18.6,18.2,17.3,15.7,11.9$. For comparison of the ${ }^{13} \mathrm{C}$ NMR spectral data of $\mathbf{4 5}$ with the natural sample, see Table 1, page S16. Reverse phase HPLC (Microsorb 100 $\mathrm{C} 18,250 \mathrm{~mm} \square 4.6 \mathrm{~mm}, \mathrm{MeOH}: \mathrm{H}_{2} \mathrm{O}=75: 15$, flow rate $1 \mathrm{ml} / \mathrm{min}, \square=254 \mathrm{~nm}$ ) of the mixture of synthetic and natural samples of natural products showed two peaks eluted with the retention times of $30.6 \mathrm{~min}$ and $33.1 \mathrm{~min}$.

\section{References:}

1. Ireland, R. E.; Highsmith, T. K.; Gegnas, L. D.; Gleason, J. L. J. Org. Chem. 1992, 57, 5071.

2. Tori, M.; Toyoda, N.; Sono, M. J. Org. Chem. 1998, 63, 306.

3. Eppley, A.W.; Totah, N.I.; Tetrahedron 1997, 53, 16545.

4. Powell, D.A.; Batey, R.A.; Org. Lett. 2002, 4, 2913.

5. Gouiffès, D.; Moreau, S.; Helbecque, N., Bernier, J. L.; Henichart, J. P.; Barbin, Y.; Laurent, D.; Verbist, J. F. Tetrahedron 1988, 44, 451. 
Table 1. Comparison of ${ }^{13} \mathrm{C}$ NMR chemical shifts of natural bistramide A, with the synthetic bistramide A (1) and the (37R)-epimer (45).

\begin{tabular}{|c|c|c|c|c|}
\hline $\begin{array}{l}\text { Natural Bistramide A, } \\
{ }^{13} \mathrm{C} \text { NMR, ppm }\end{array}$ & $\begin{array}{c}\text { (37R)-epimer (45), } \\
{ }^{13} \mathrm{C} \text { NMR, ppm }\end{array}$ & $\begin{array}{c}\square, \mathrm{ppm} \\
\text { (natural - 45) }\end{array}$ & $\begin{array}{l}\text { Synthetic Bistramide A } \\
(\mathbf{1}),{ }^{13} \mathrm{C} \text { NMR, ppm }\end{array}$ & $\begin{array}{c}\begin{array}{c}\square, \mathrm{ppm} \\
\text { (natural - 1) }\end{array} \\
\end{array}$ \\
\hline 199.1 & 199.117 & -0.017 & 199.124 & -0.024 \\
\hline 175.307 & 175.364 & -0.057 & 175.316 & -0.009 \\
\hline 173.663 & 173.626 & 0.037 & 173.669 & -0.006 \\
\hline 144.699 & 144.723 & -0.024 & 144.728 & -0.029 \\
\hline 137.316 & 137.408 & -0.092 & 137.308 & 0.008 \\
\hline 132.253 & 132.252 & 0.001 & 132.246 & 0.007 \\
\hline 131.53 & 131.545 & -0.015 & 131.522 & 0.008 \\
\hline 95.6093 & 95.6423 & -0.033 & 95.6088 & 0.0005 \\
\hline 74.9916 & 74.9895 & 0.0021 & 74.9872 & 0.0044 \\
\hline 74.4089 & 74.2947 & 0.1142 & 74.4029 & 0.006 \\
\hline 74.0087 & 73.9905 & 0.0182 & 73.985 & 0.0237 \\
\hline 73.4414 & 73.4883 & -0.0469 & 73.435 & 0.0064 \\
\hline 69.2287 & 69.4059 & -0.1772 & 69.2231 & 0.0056 \\
\hline 64.9036 & 64.9048 & -0.0012 & 64.894 & 0.0096 \\
\hline 45.4054 & 45.4076 & -0.0022 & 45.3947 & 0.0107 \\
\hline 44.838 & 44.7887 & 0.0493 & 44.8236 & 0.0144 \\
\hline 43.4919 & 43.4229 & 0.069 & 43.4827 & 0.0092 \\
\hline 39.6396 & 39.5067 & 0.1329 & 39.6326 & 0.007 \\
\hline 36.2388 & 36.2391 & -0.0003 & 36.2337 & 0.0051 \\
\hline 35.6239 & 35.6351 & -0.0112 & 35.6199 & 0.004 \\
\hline 35.0299 & 35.0986 & -0.0687 & 35.0291 & 0.0008 \\
\hline 34.2444 & 34.1729 & 0.0715 & 34.2405 & 0.0039 \\
\hline 33.6261 & 33.6557 & -0.0296 & 33.6223 & 0.0038 \\
\hline 33.4807 & 33.4798 & 0.0009 & 33.4755 & 0.0052 \\
\hline 32.4355 & 32.4331 & 0.0024 & 32.4223 & 0.0132 \\
\hline 32.017 & 32.0204 & -0.0034 & 32.0141 & 0.0029 \\
\hline 31.4919 & 31.4251 & 0.0668 & 31.4863 & 0.0056 \\
\hline 30.9482 & 30.9562 & -0.008 & 30.9482 & 0 \\
\hline 30.5823 & 30.4338 & 0.1485 & 30.571 & 0.0113 \\
\hline 28.0476 & 28.081 & -0.0334 & 28.0419 & 0.0057 \\
\hline 26.6554 & 26.6523 & 0.0031 & 26.6488 & 0.0066 \\
\hline 26.0206 & 25.987 & 0.0336 & 26.0175 & 0.0031 \\
\hline 21.881 & 21.809 & 0.072 & 21.872 & 0.009 \\
\hline 21.1148 & 21.0015 & 0.1133 & 21.1177 & -0.0029 \\
\hline 19.3678 & 19.3859 & -0.0181 & 19.3608 & 0.007 \\
\hline 18.5772 & 18.5836 & -0.0064 & 18.5846 & -0.0074 \\
\hline 18.1593 & 18.1549 & 0.0044 & 18.1586 & 0.0007 \\
\hline 17.3099 & 17.3119 & -0.002 & 17.3122 & -0.0023 \\
\hline 15.7112 & 15.7371 & -0.0259 & 15.7117 & -0.0005 \\
\hline 11.9491 & 11.8485 & 0.1006 & 11.9479 & 0.0012 \\
\hline
\end{tabular}




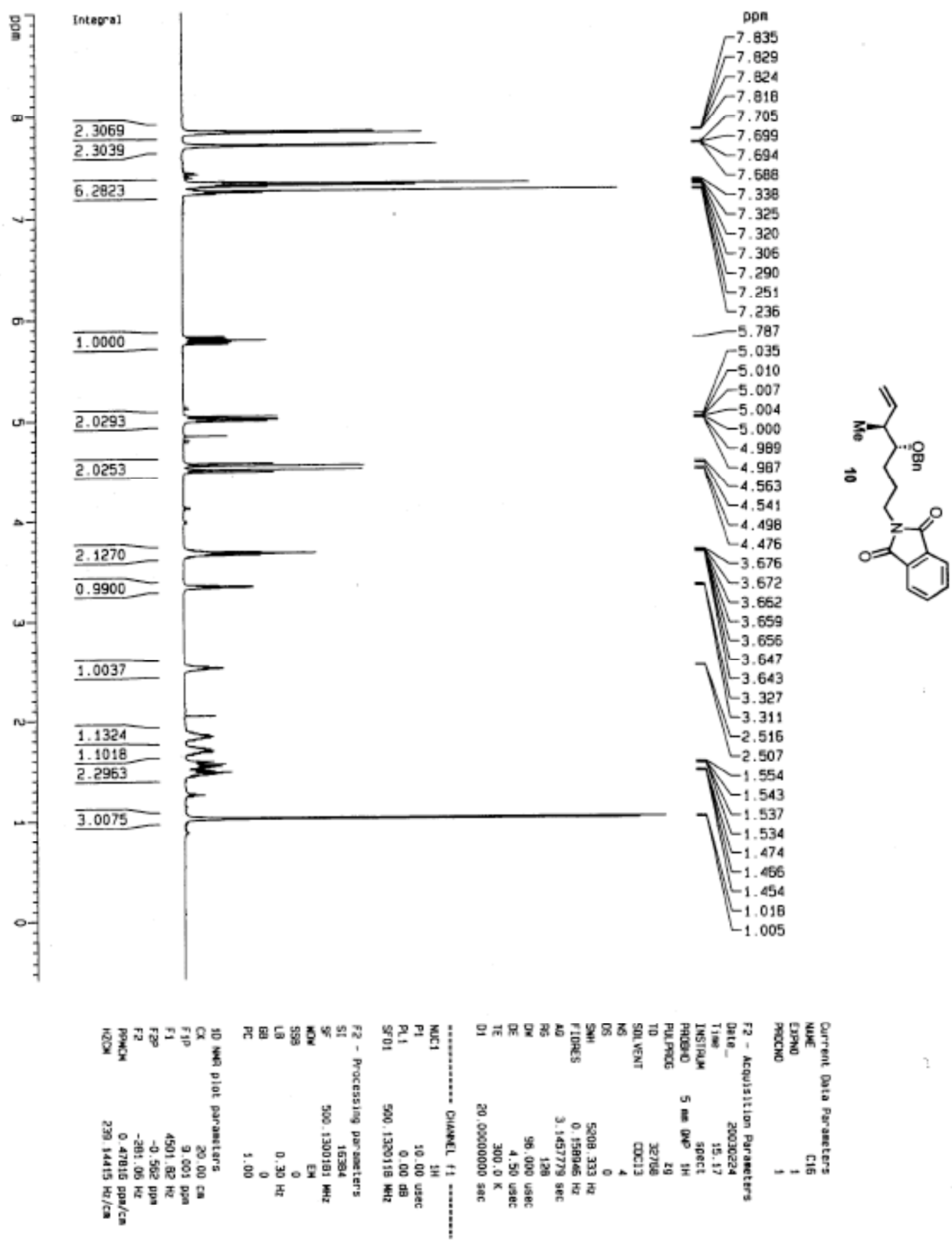




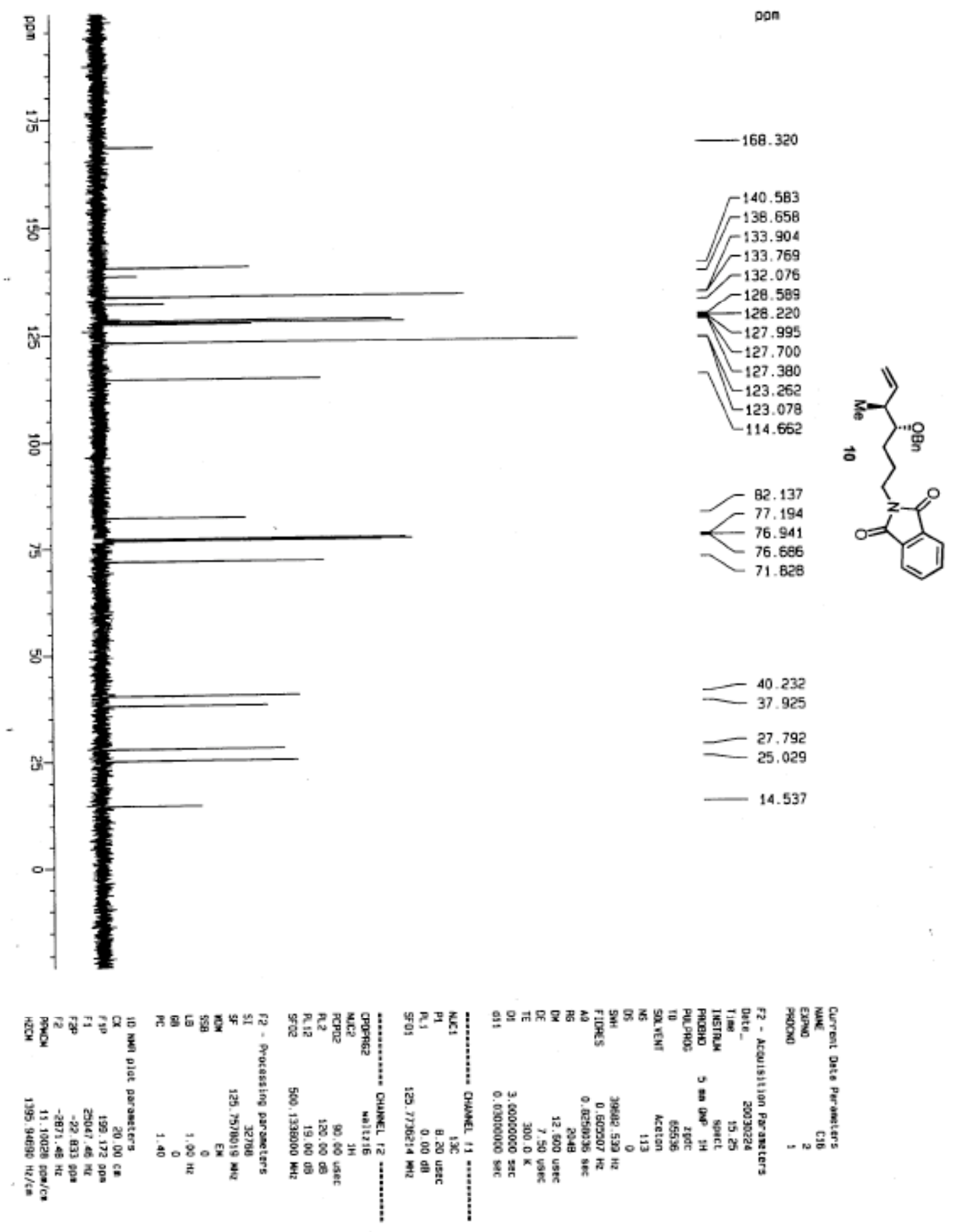




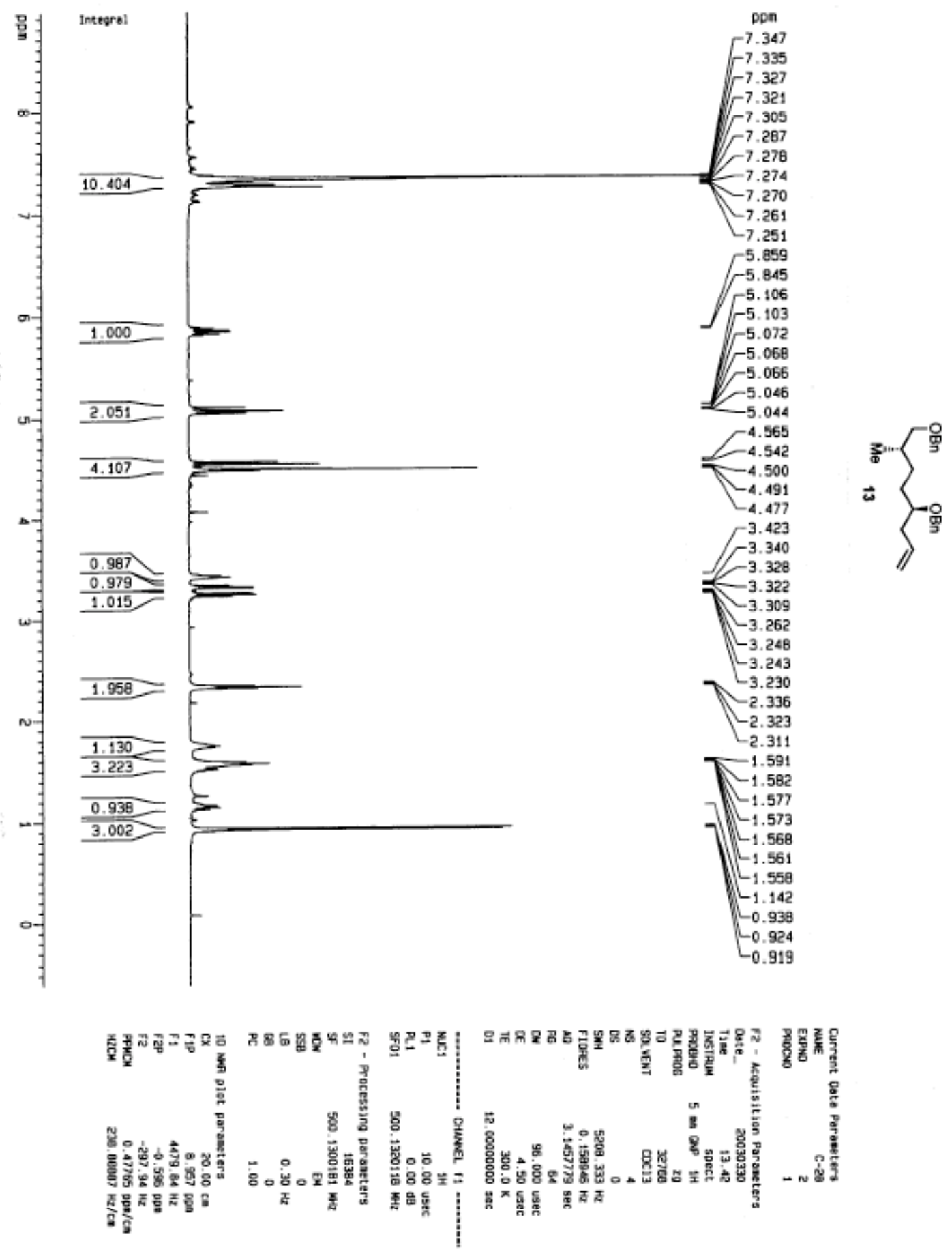




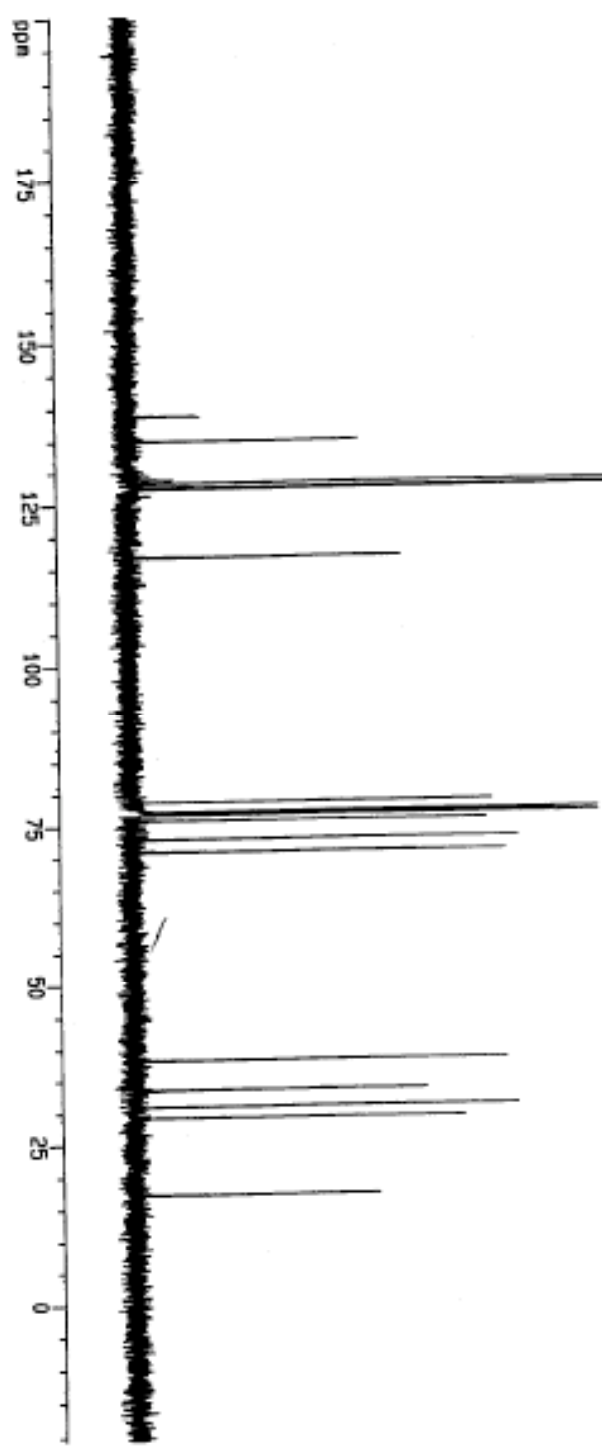

138.824

${ }_{-138.708}^{-138.824}$

$-134.931$

$-128.751$

128.225
-127.710

$-127.633$

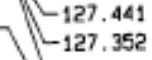

$L_{127.336}$

$-116.765$

78.713

F 77.713

76.939

76.683

$\begin{array}{r}-75.737 \\ \hline\end{array}$

$-72.888$

$-70.808$

$-38.087$

33.462

29.158

17,121

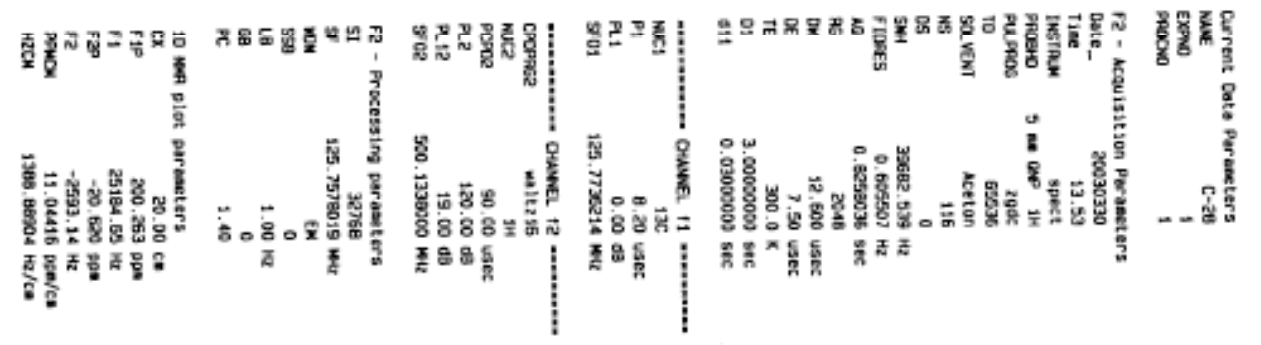




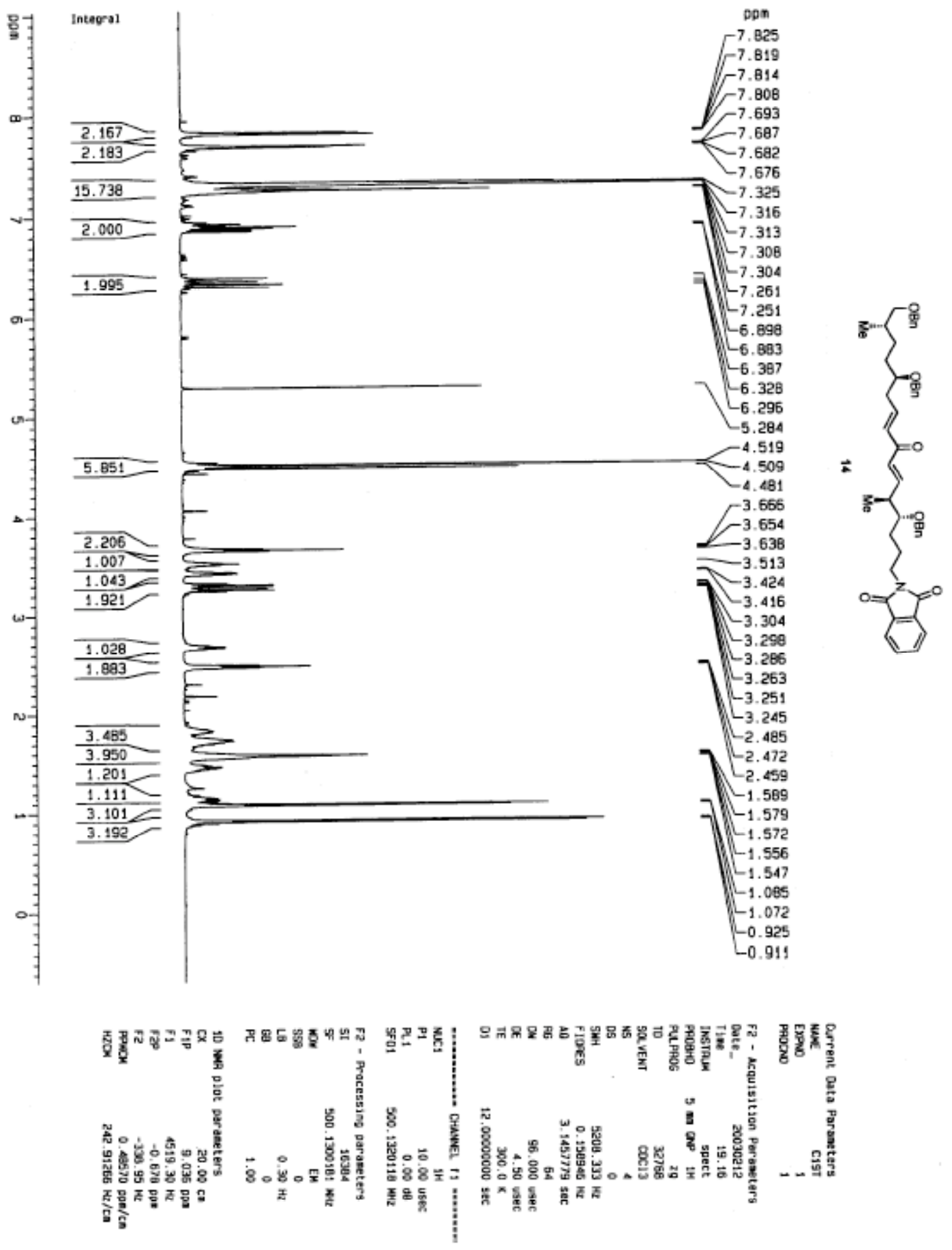




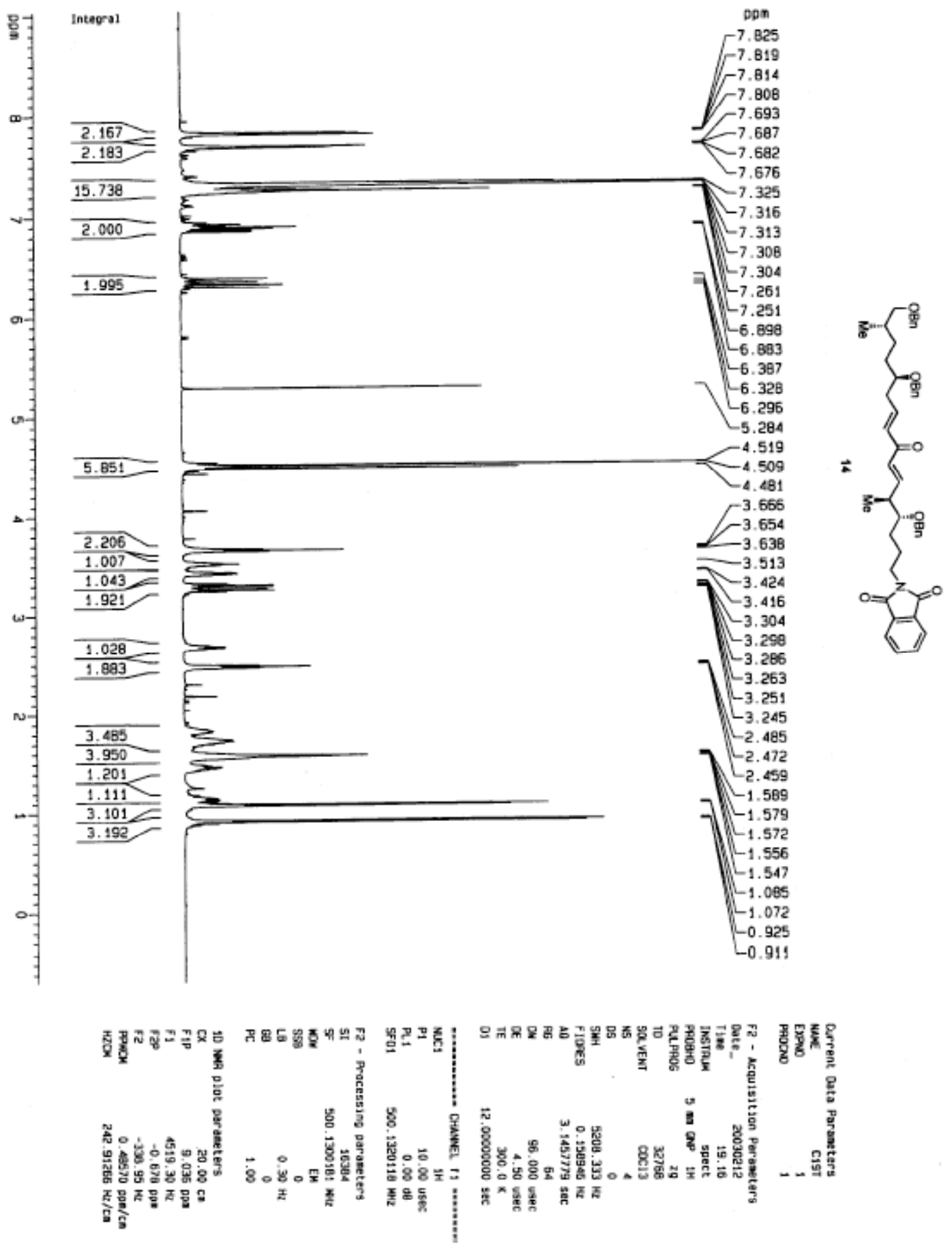




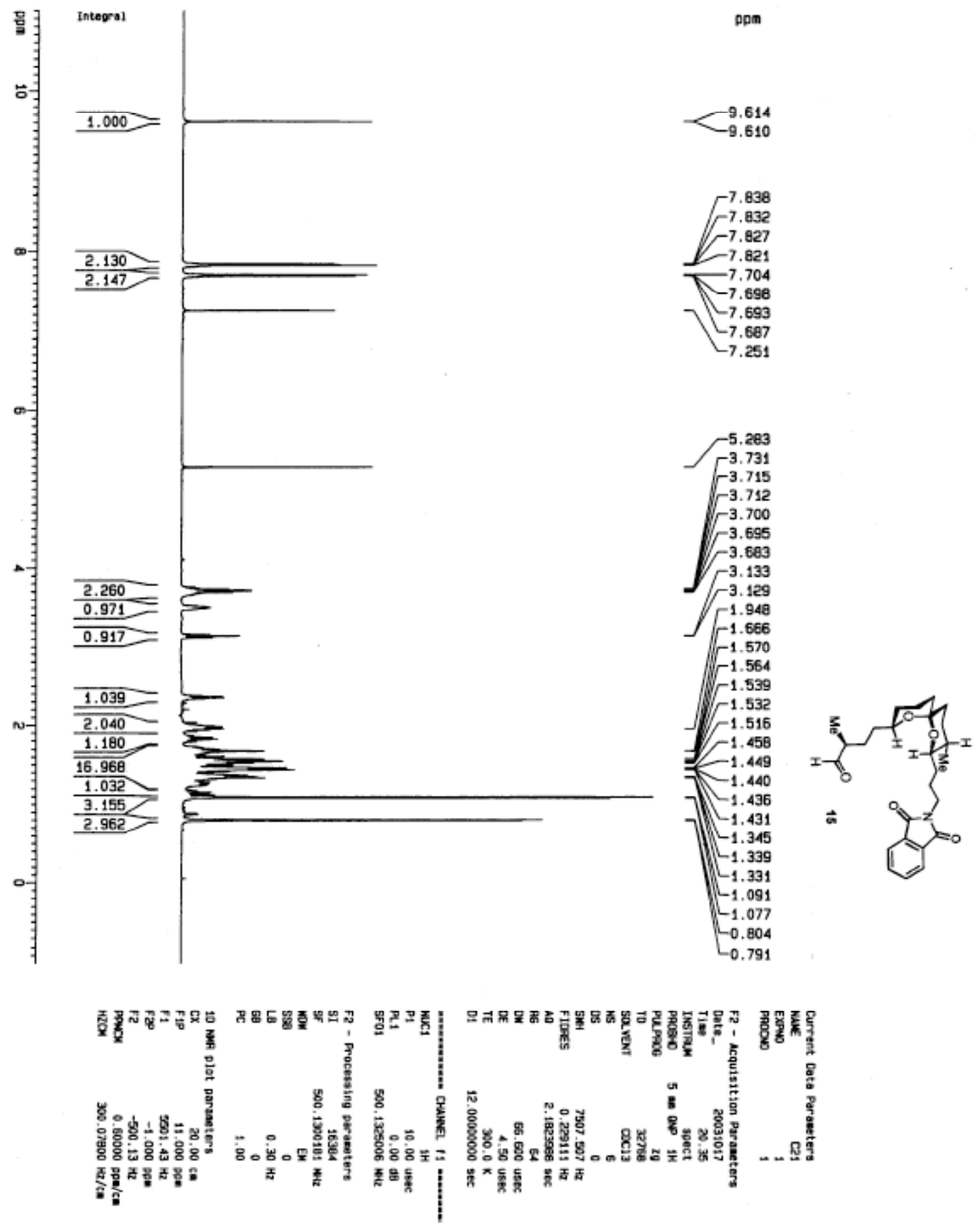




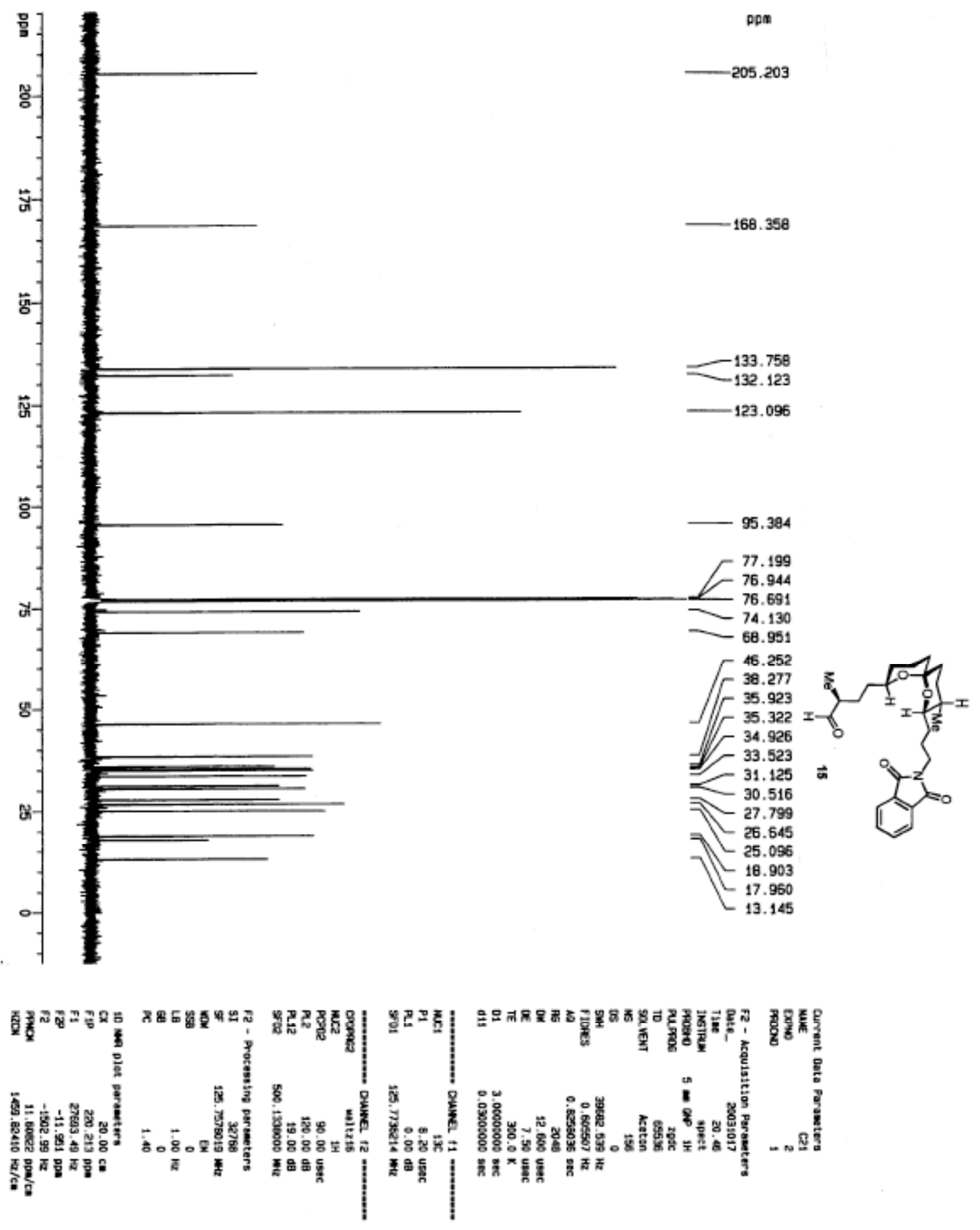




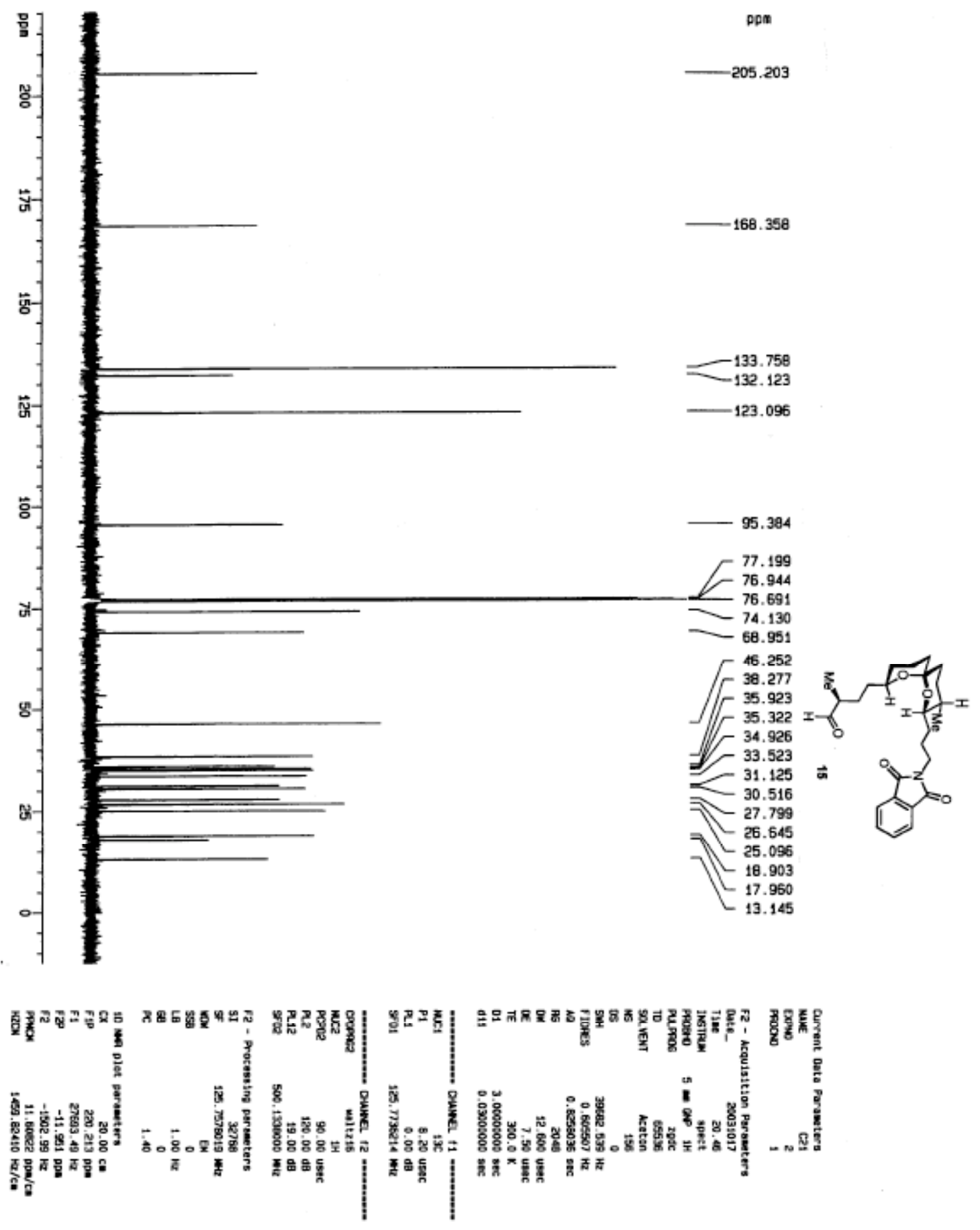




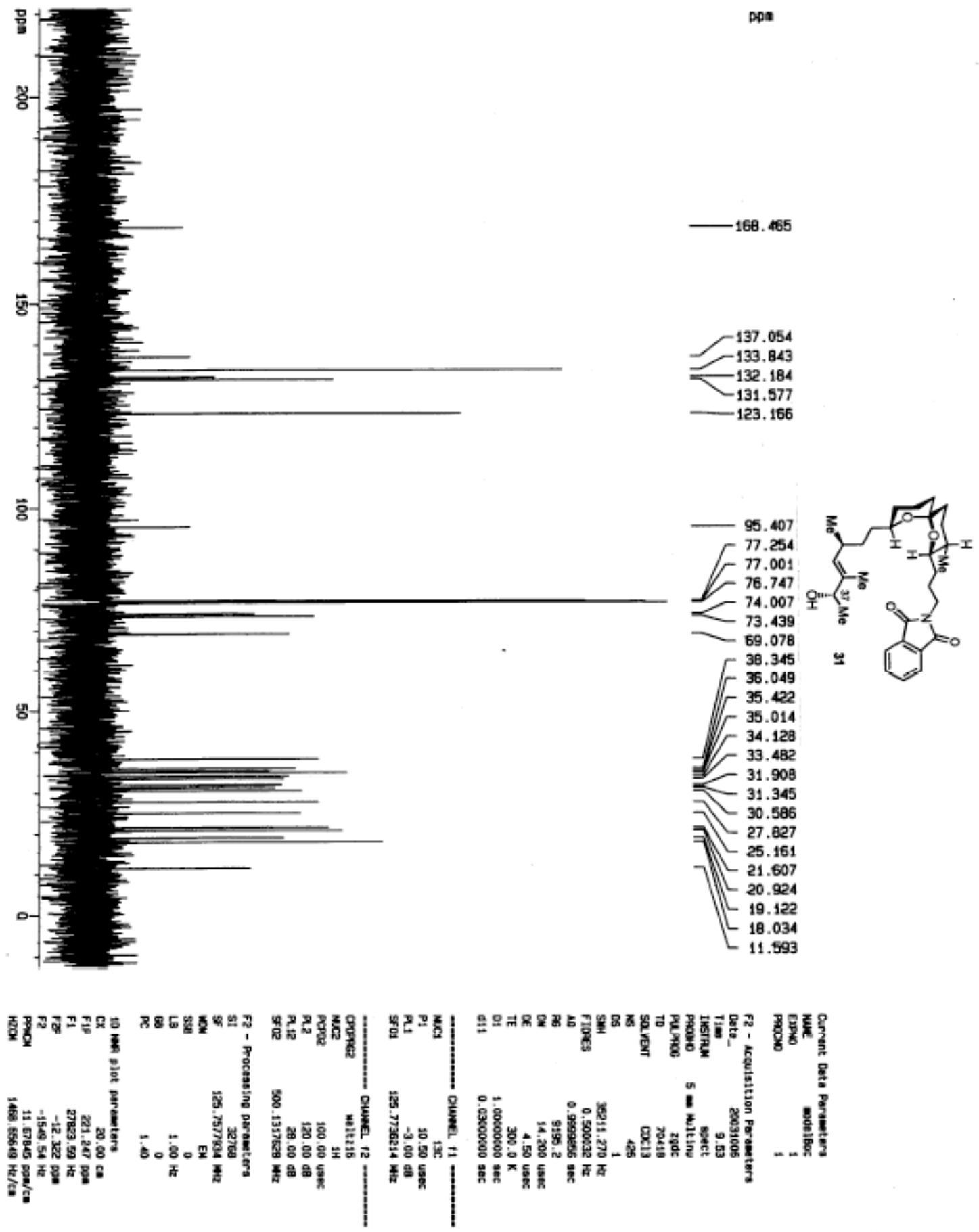




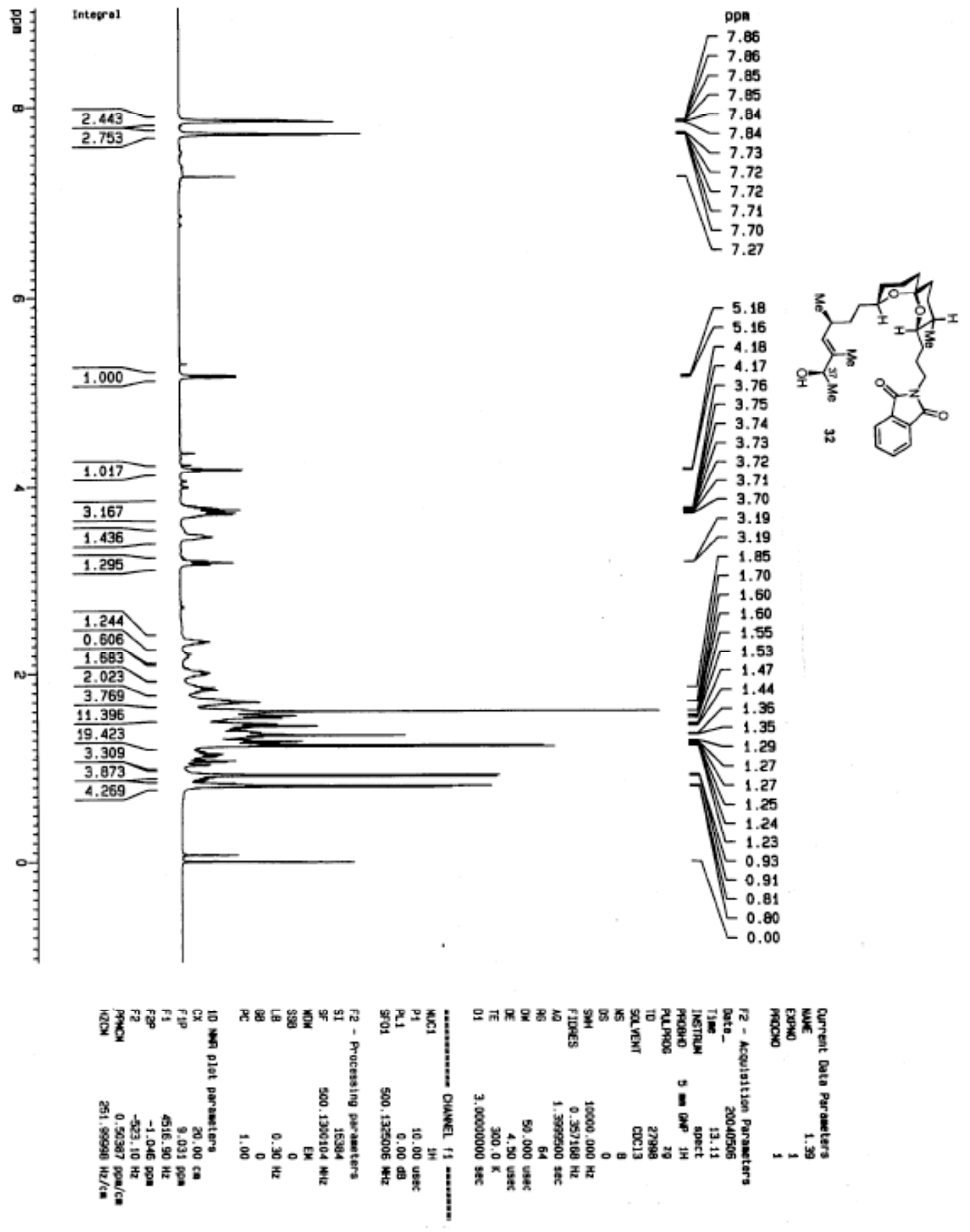




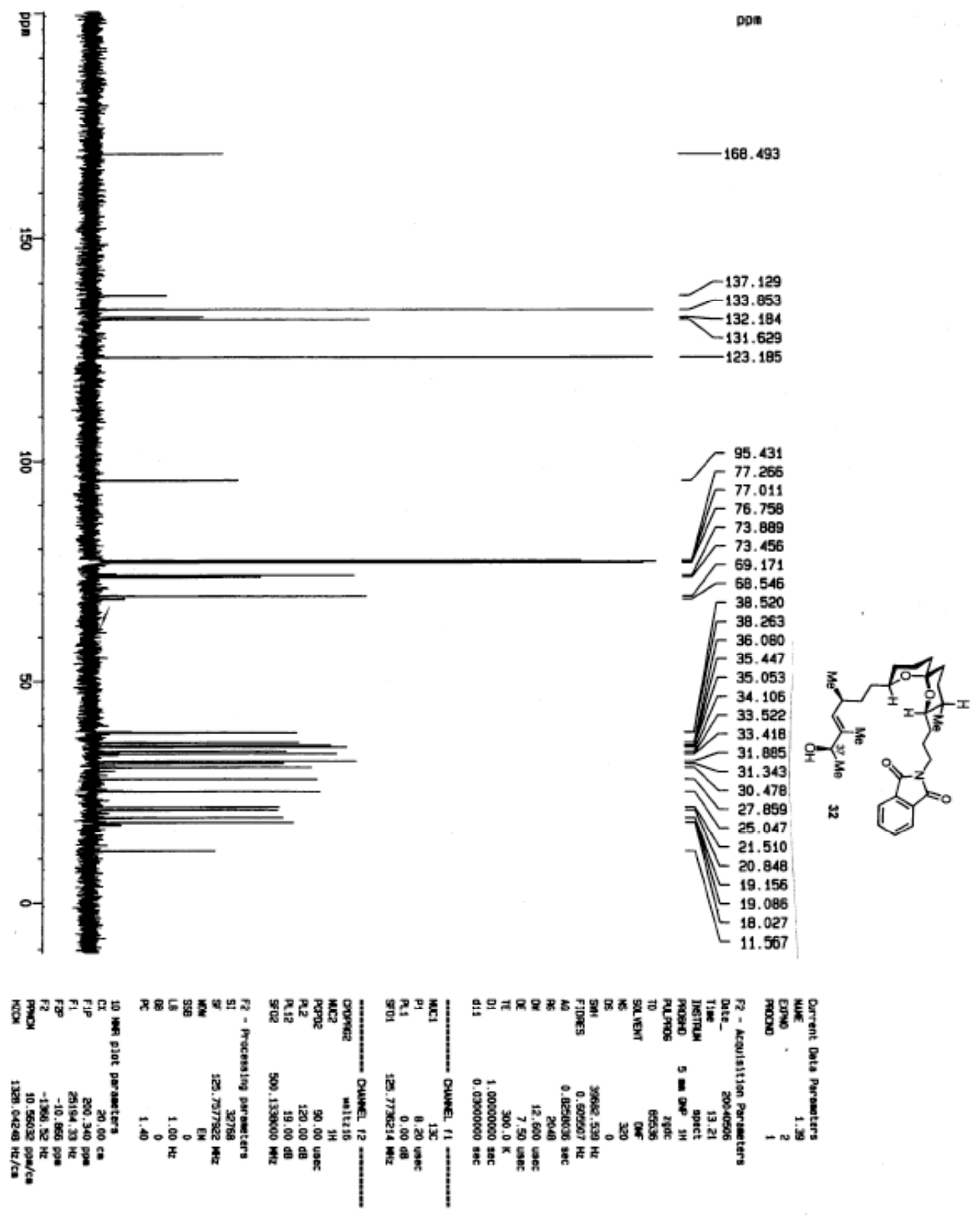




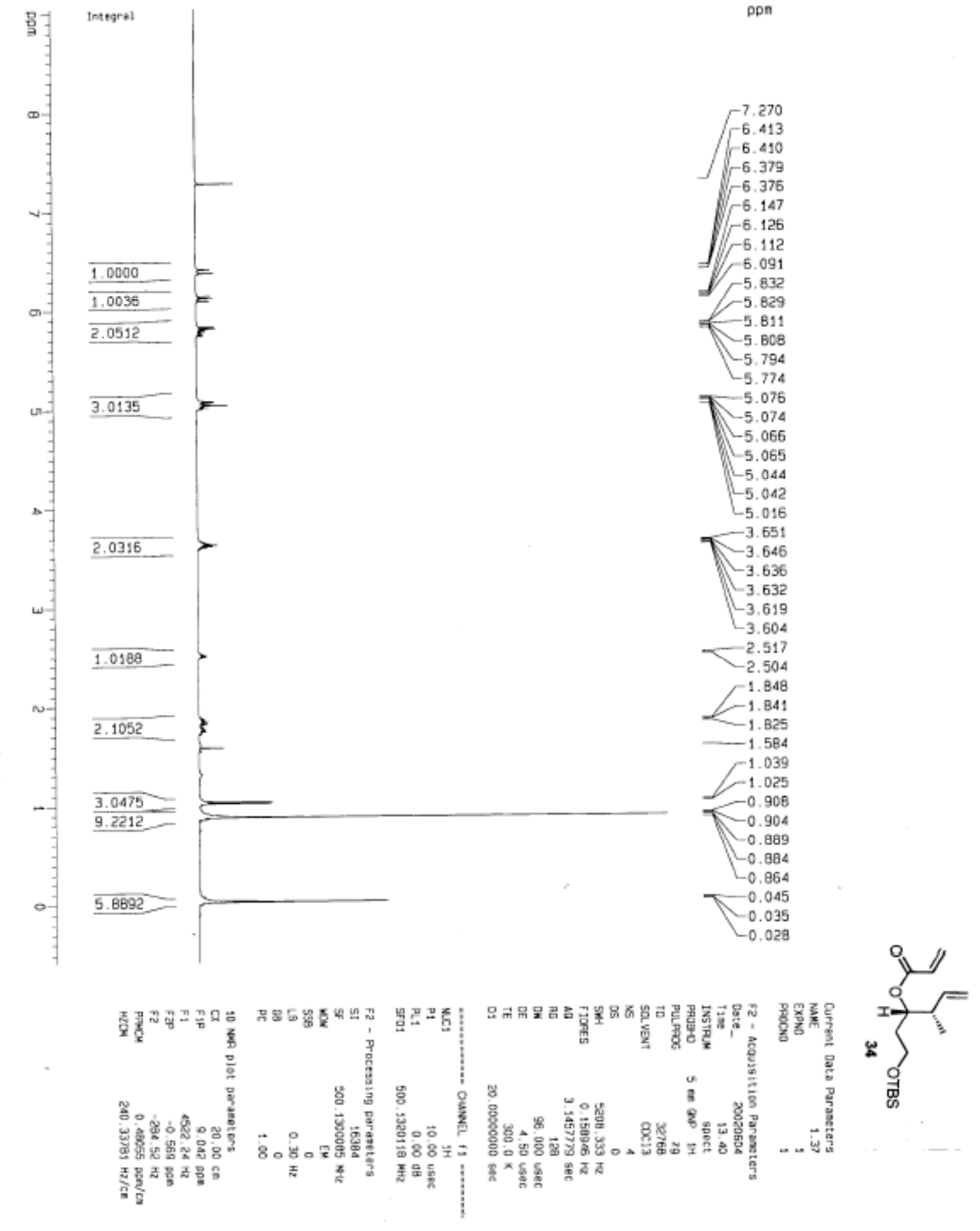




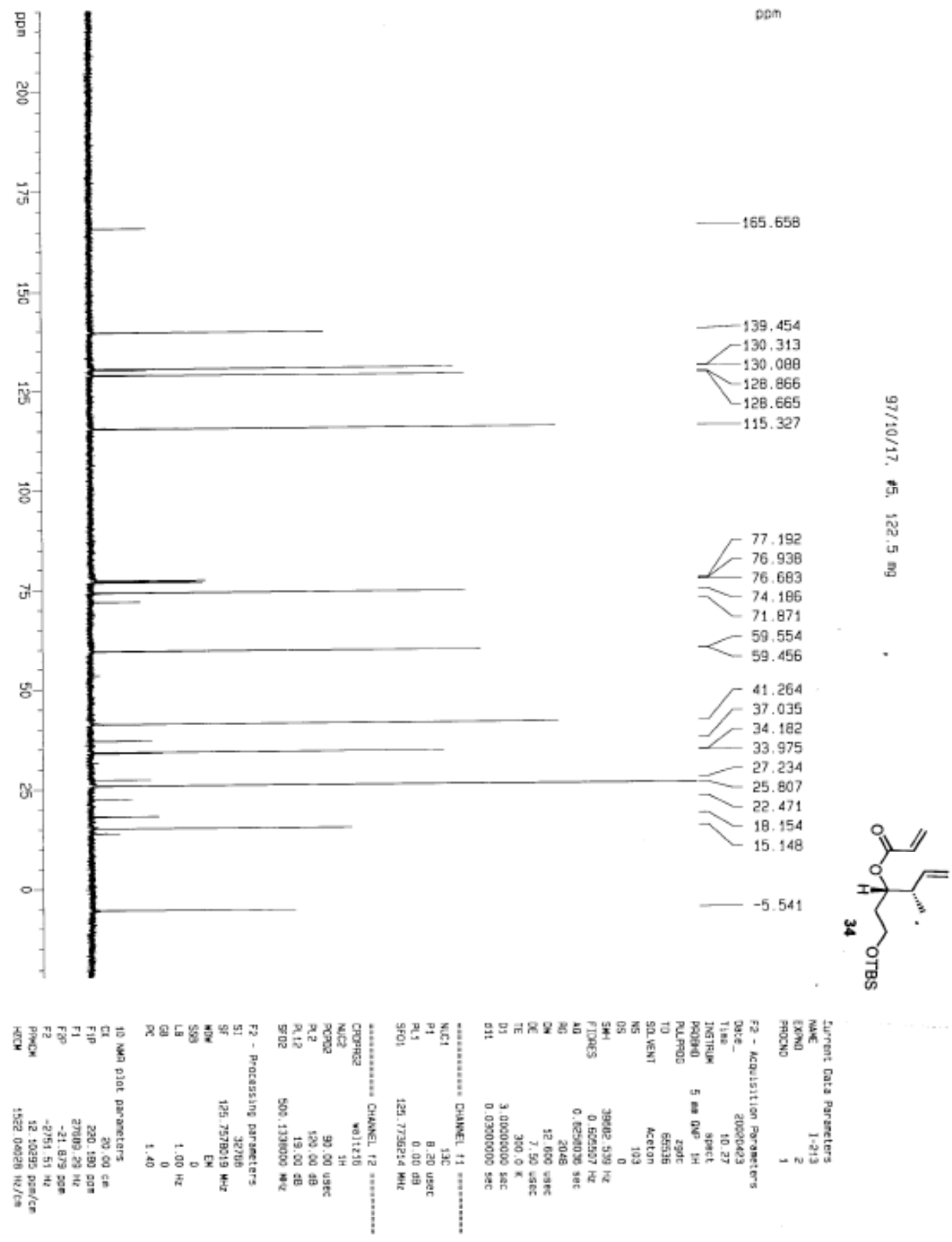




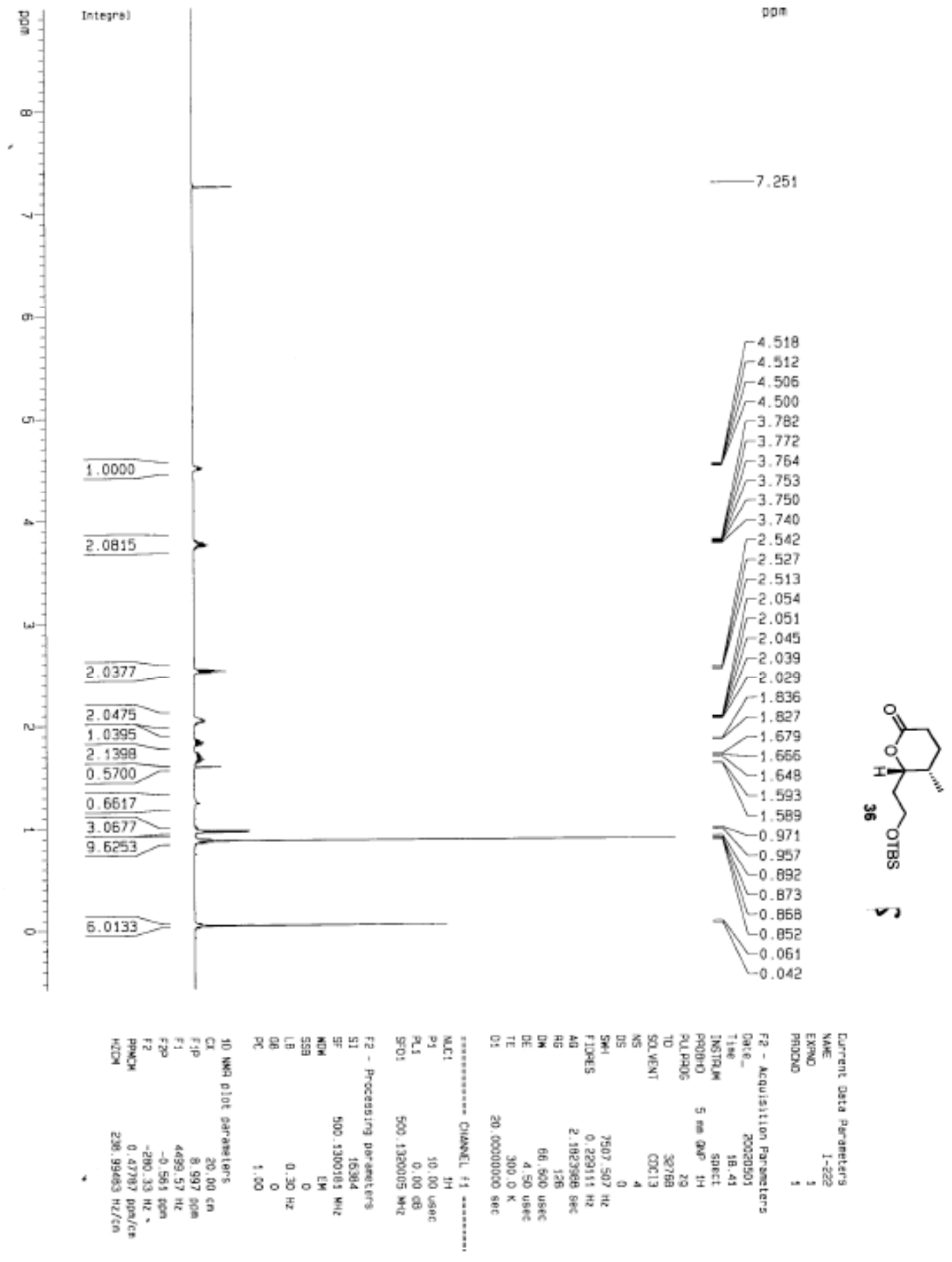




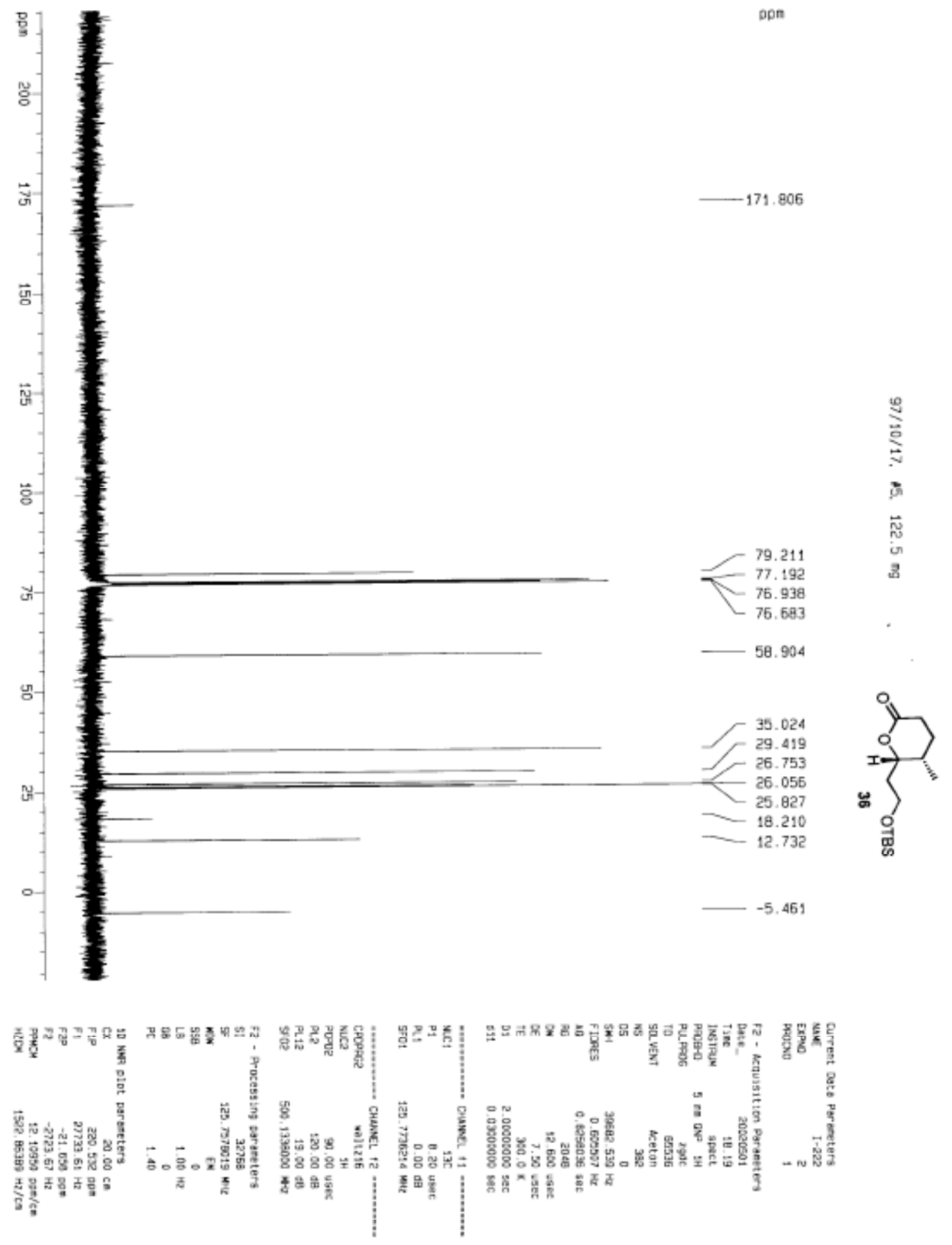




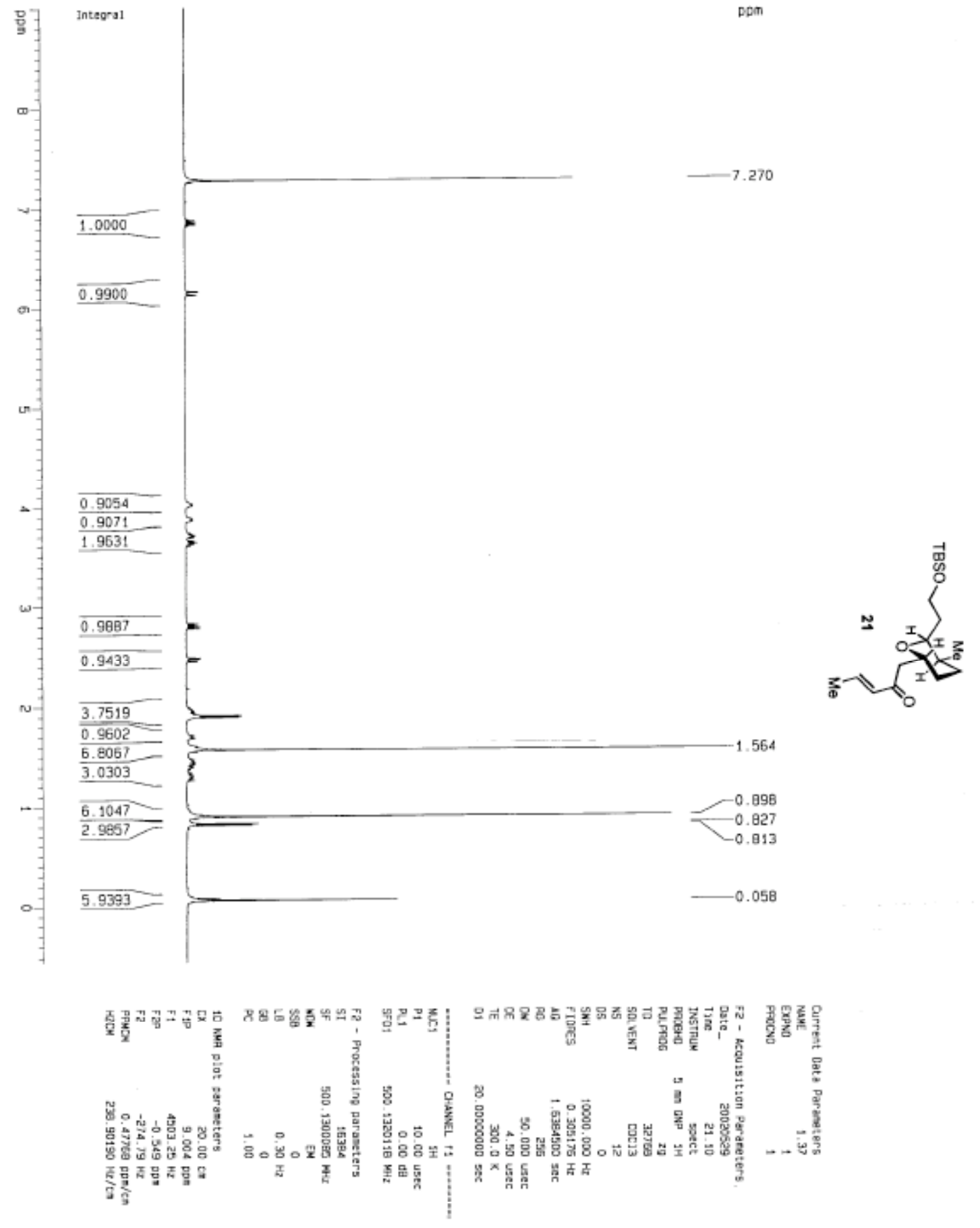




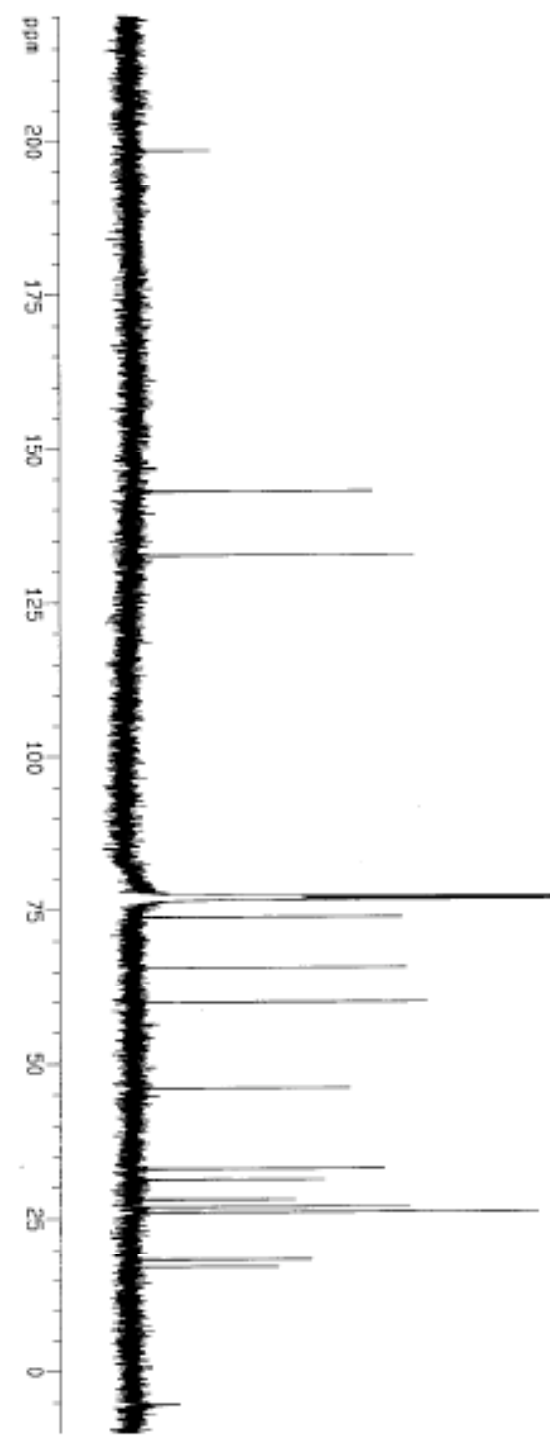




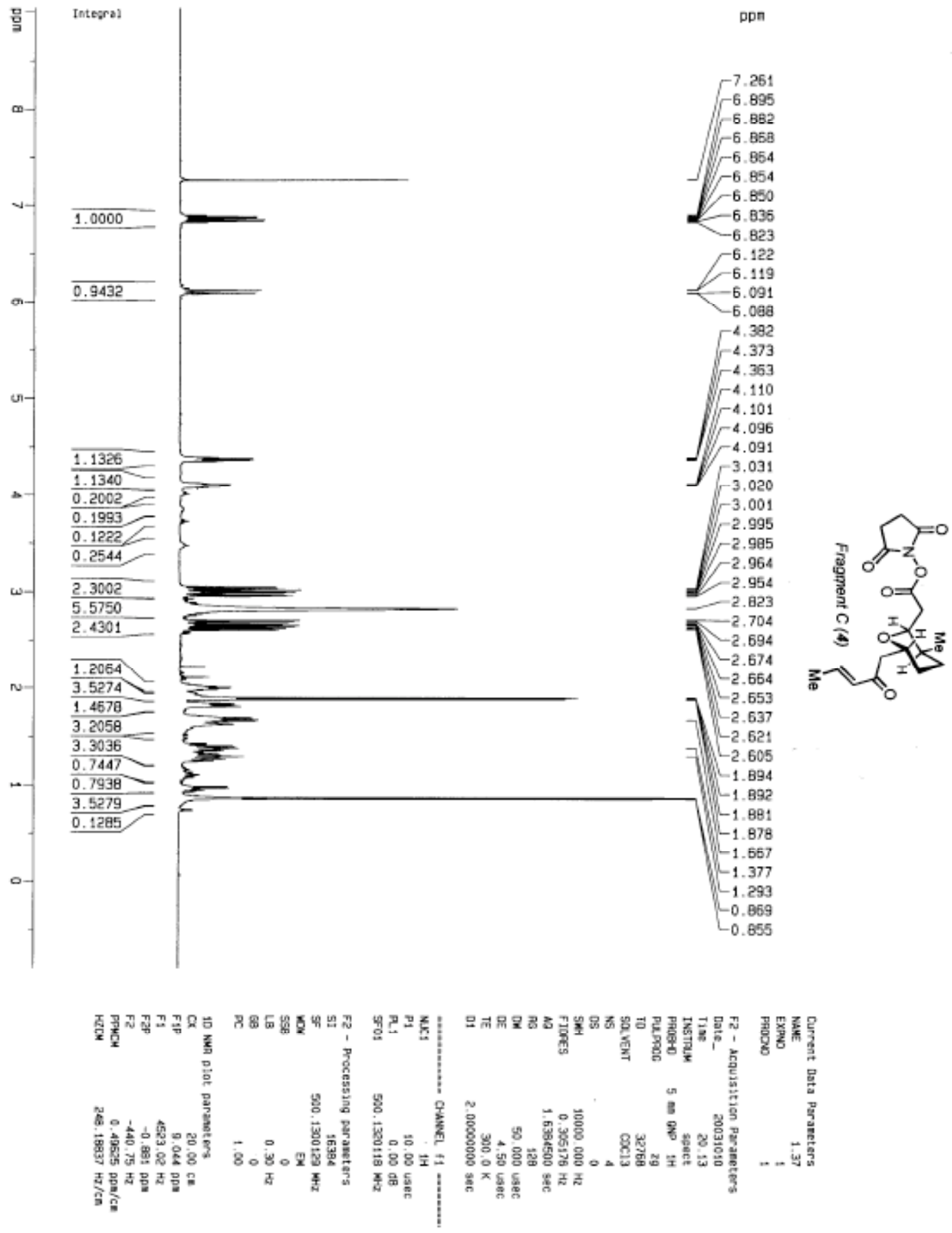




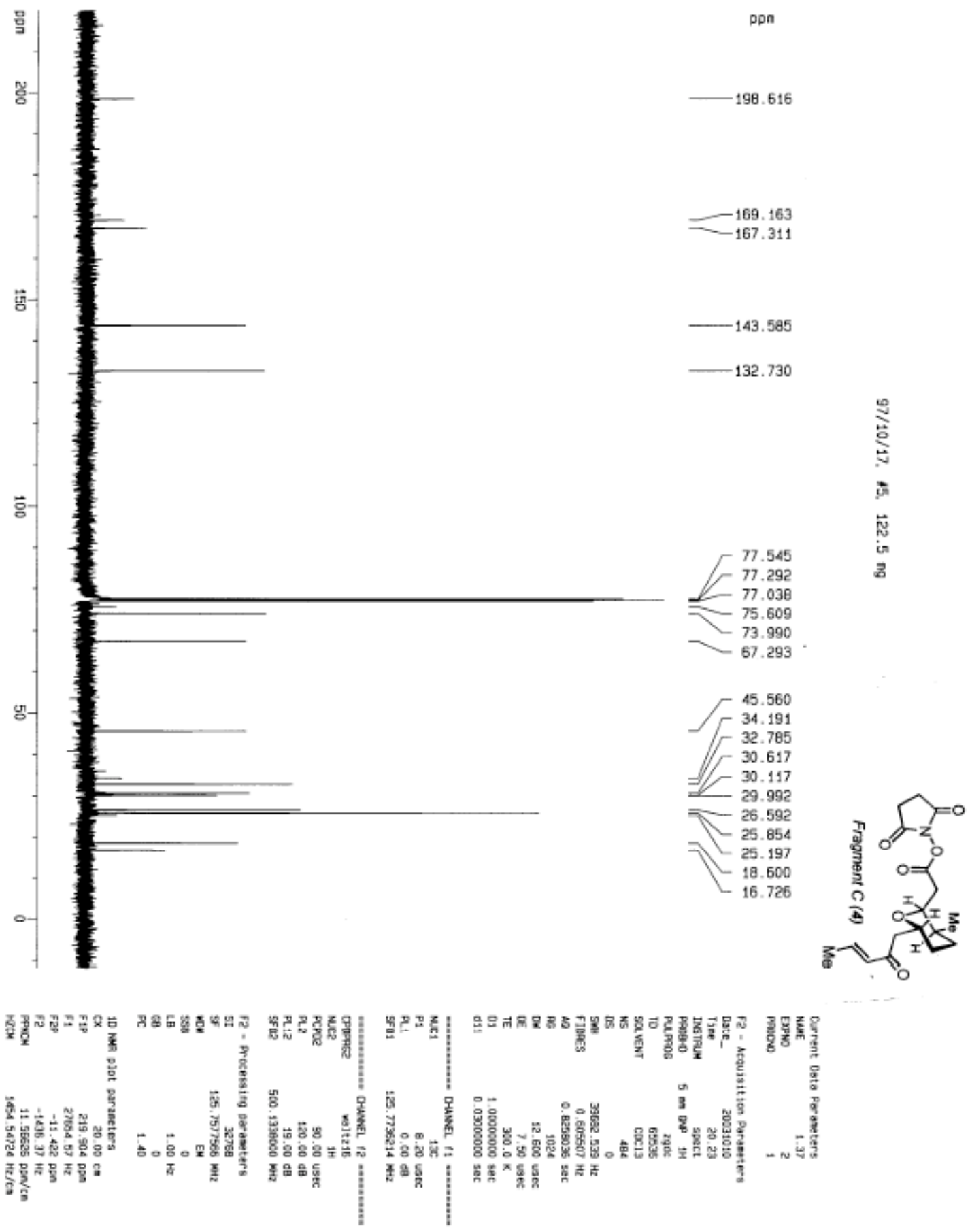




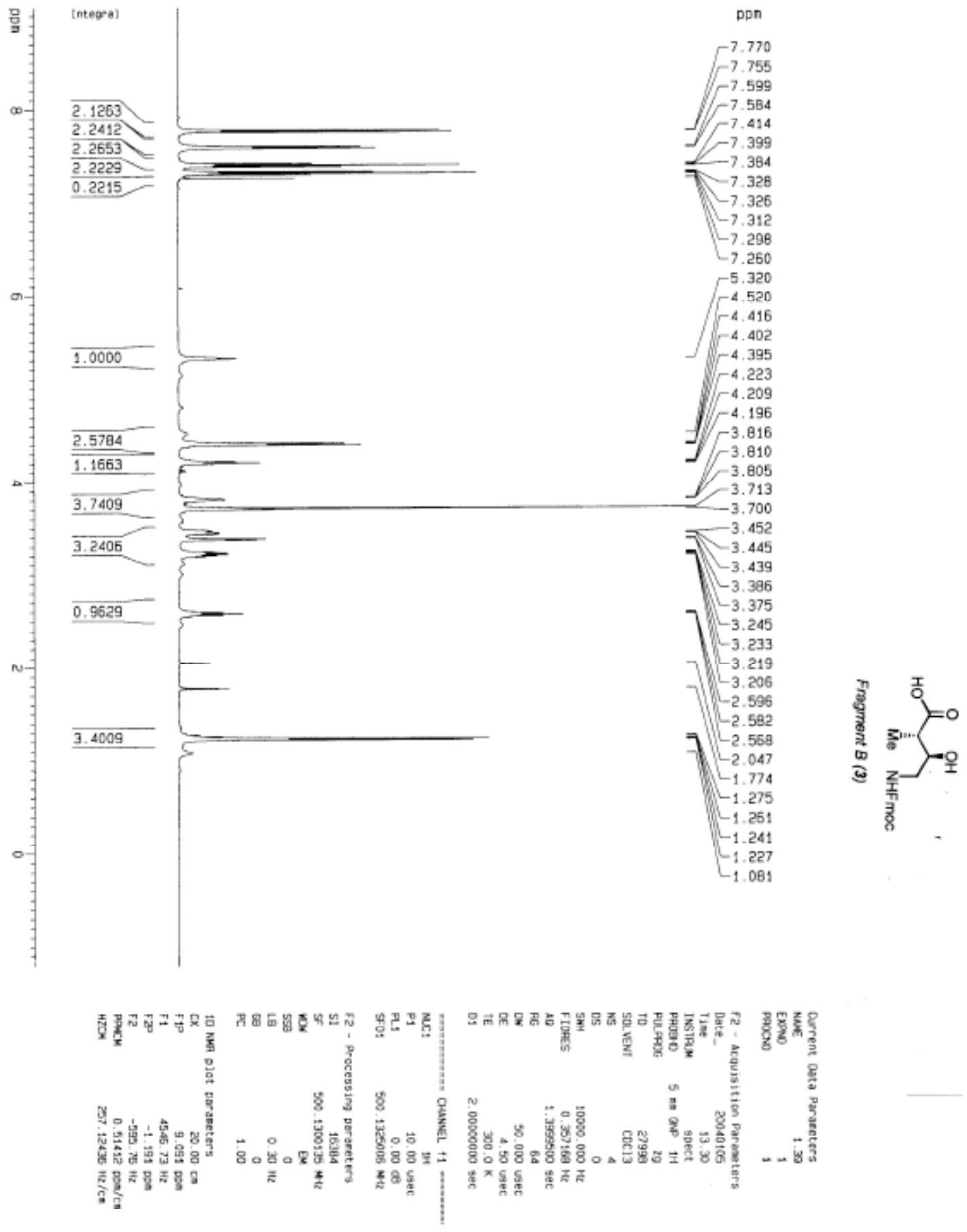



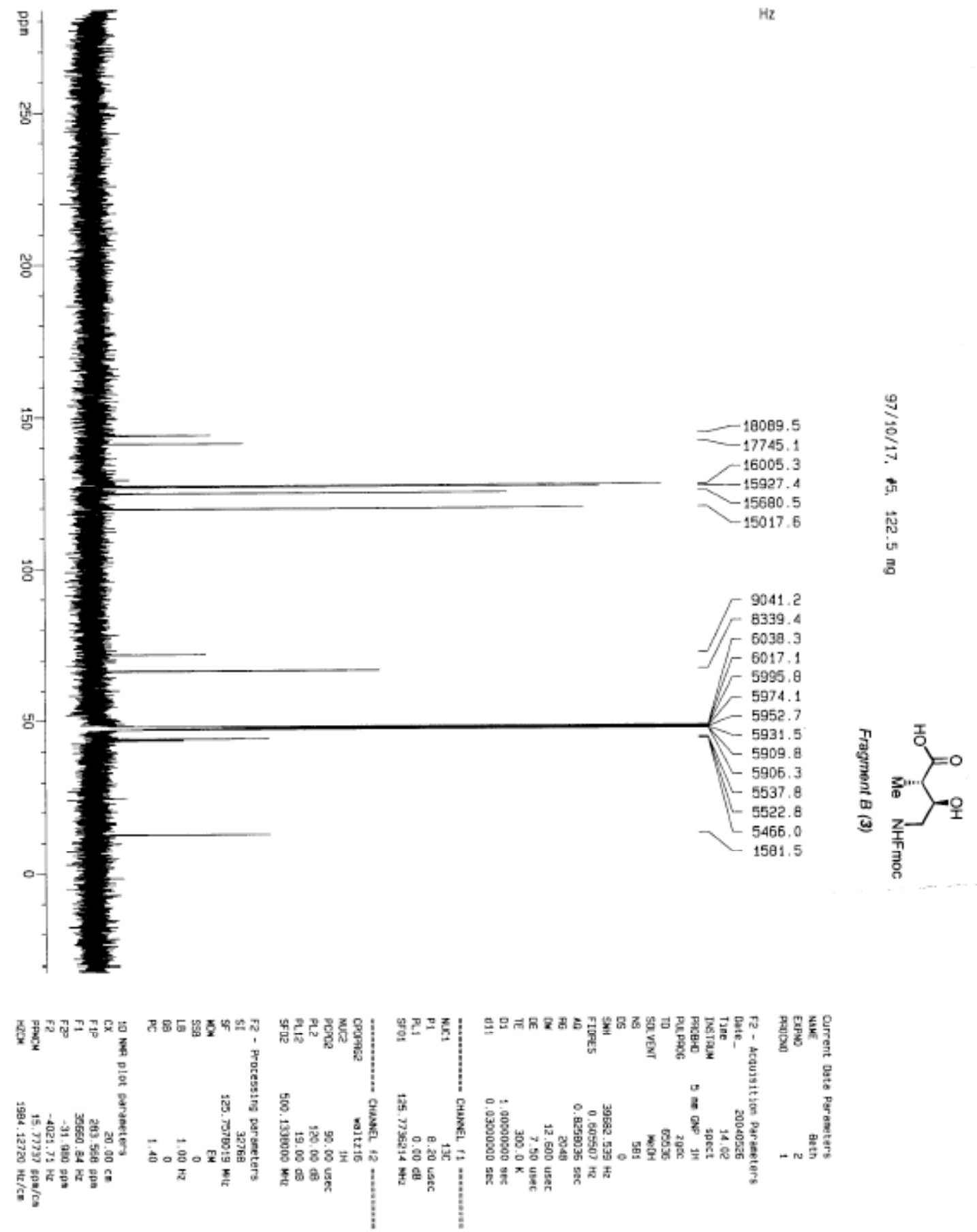


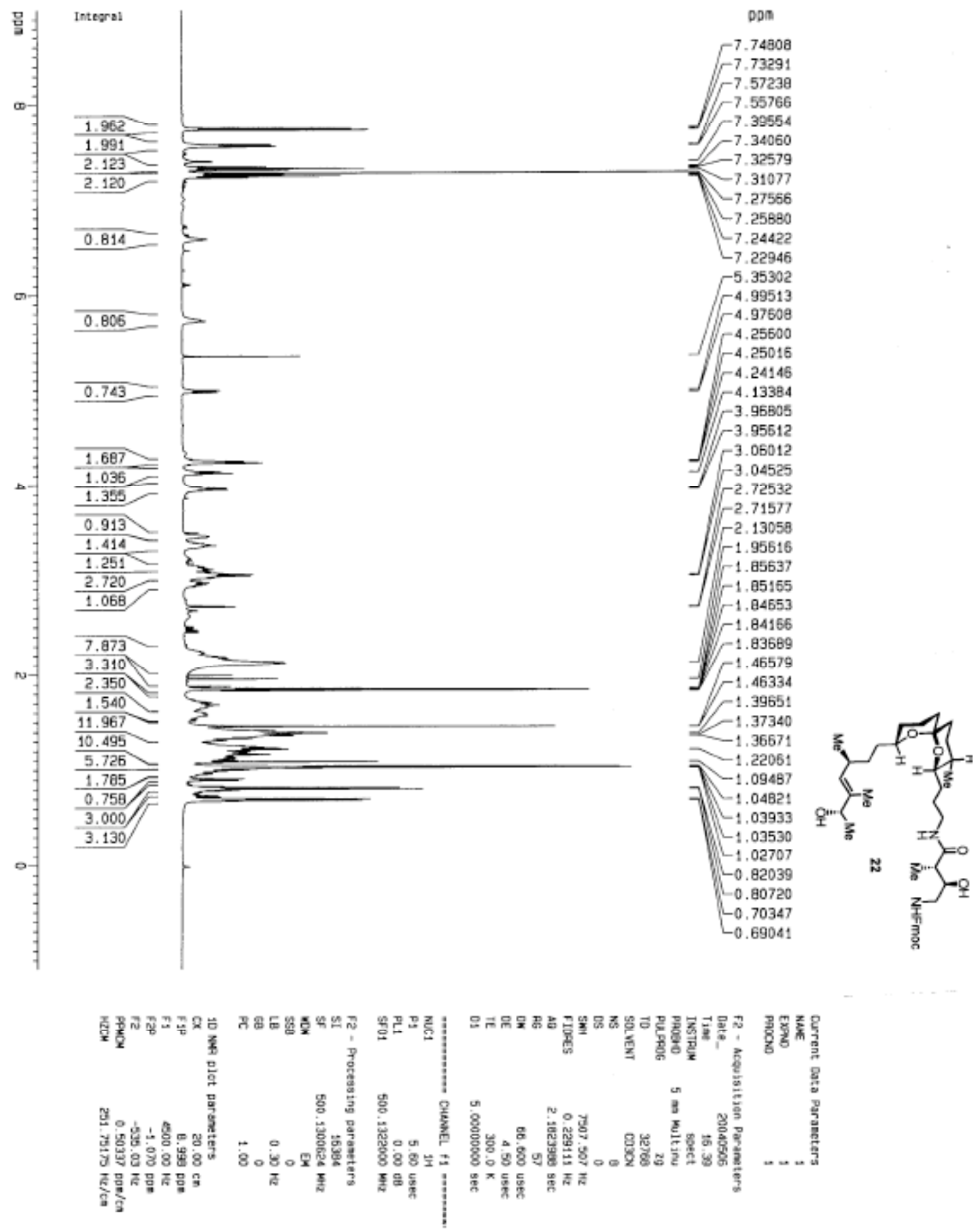




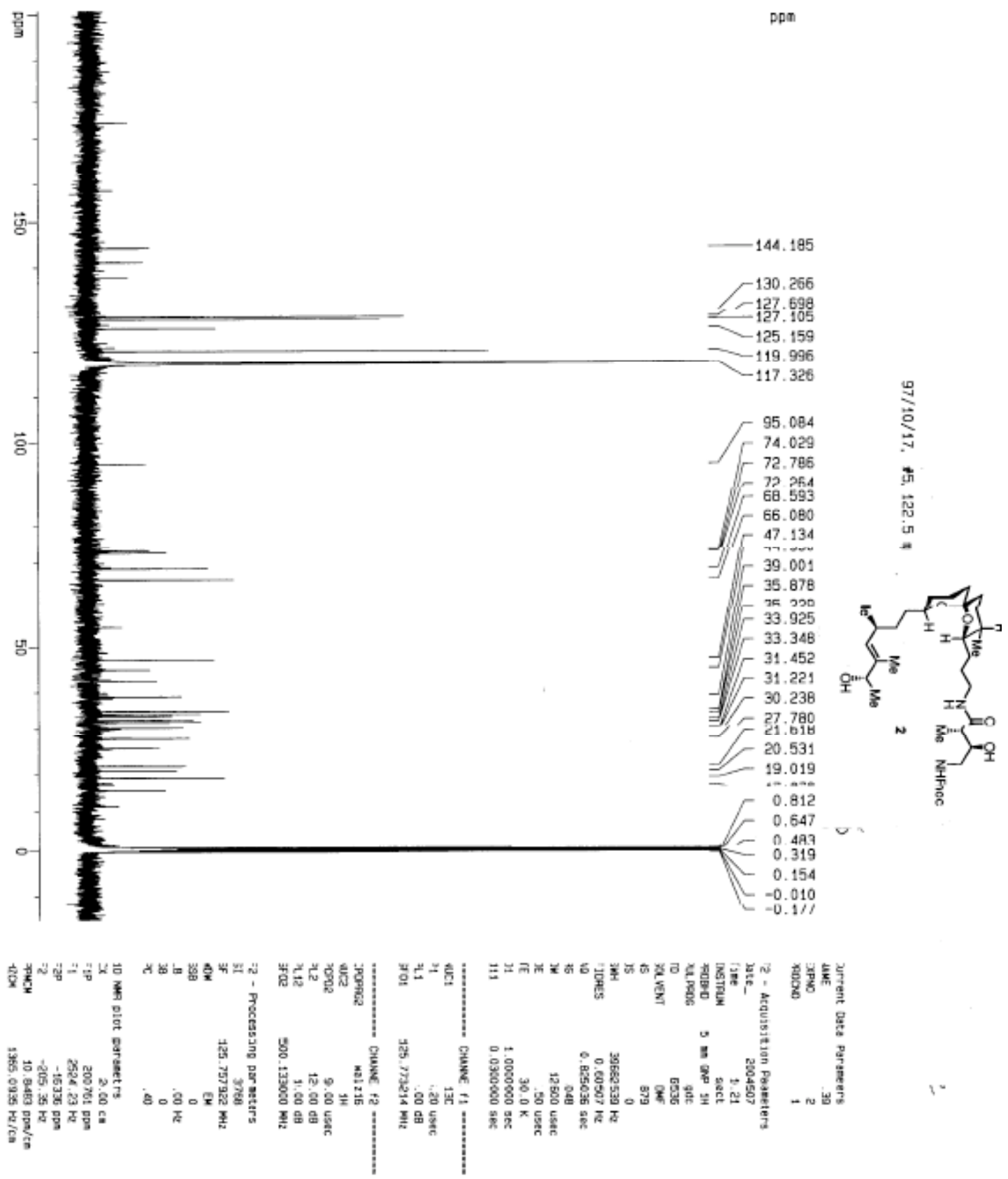




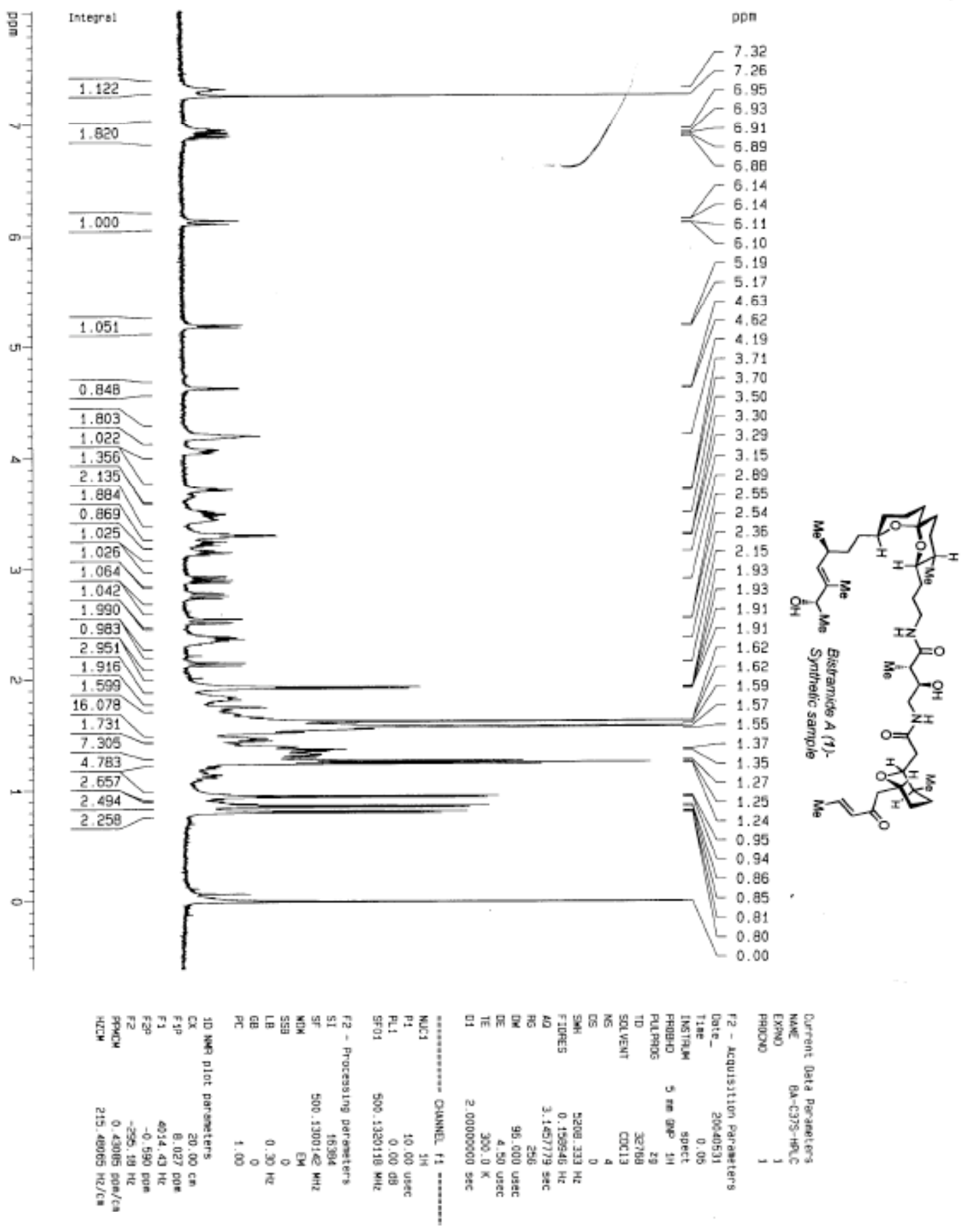




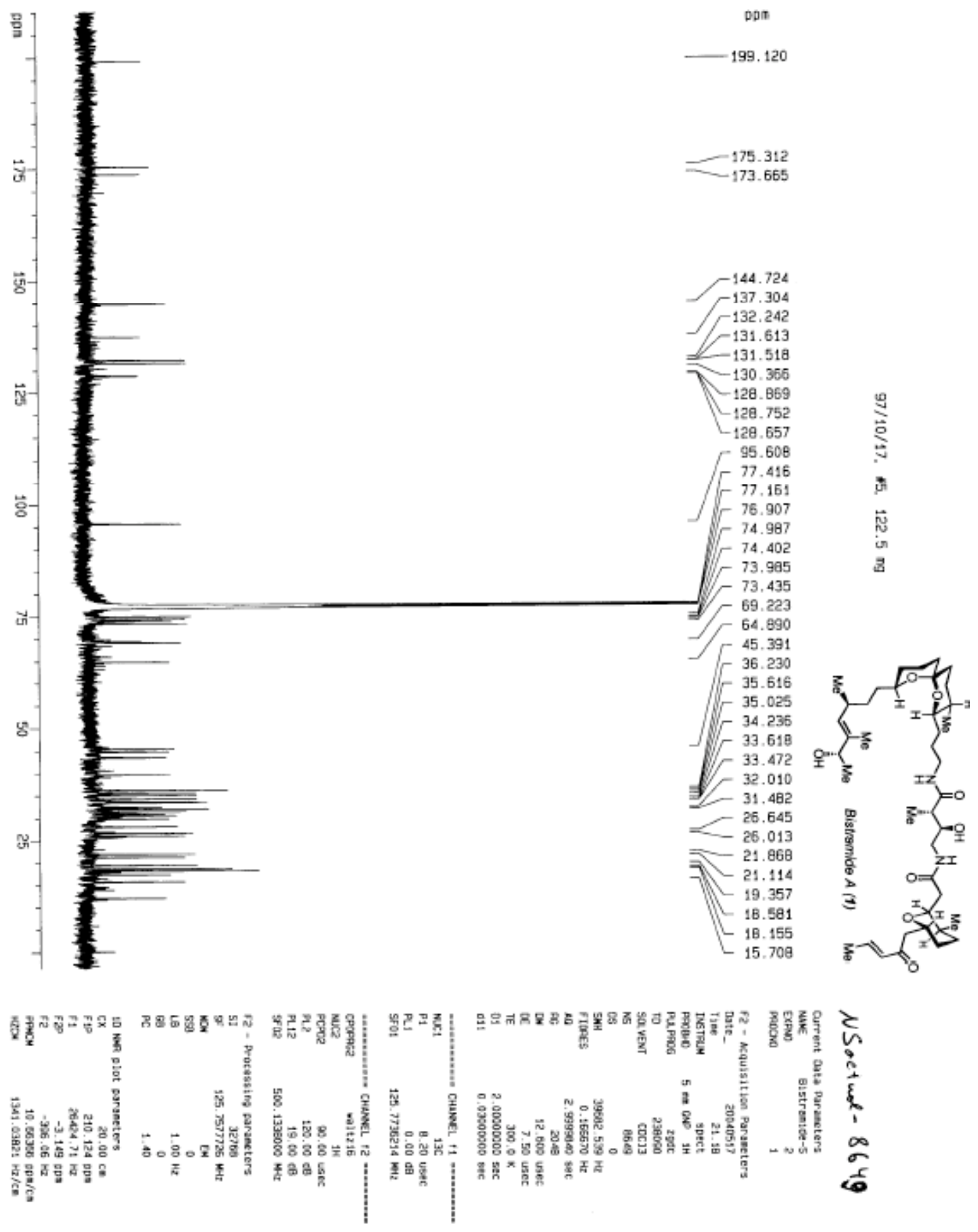




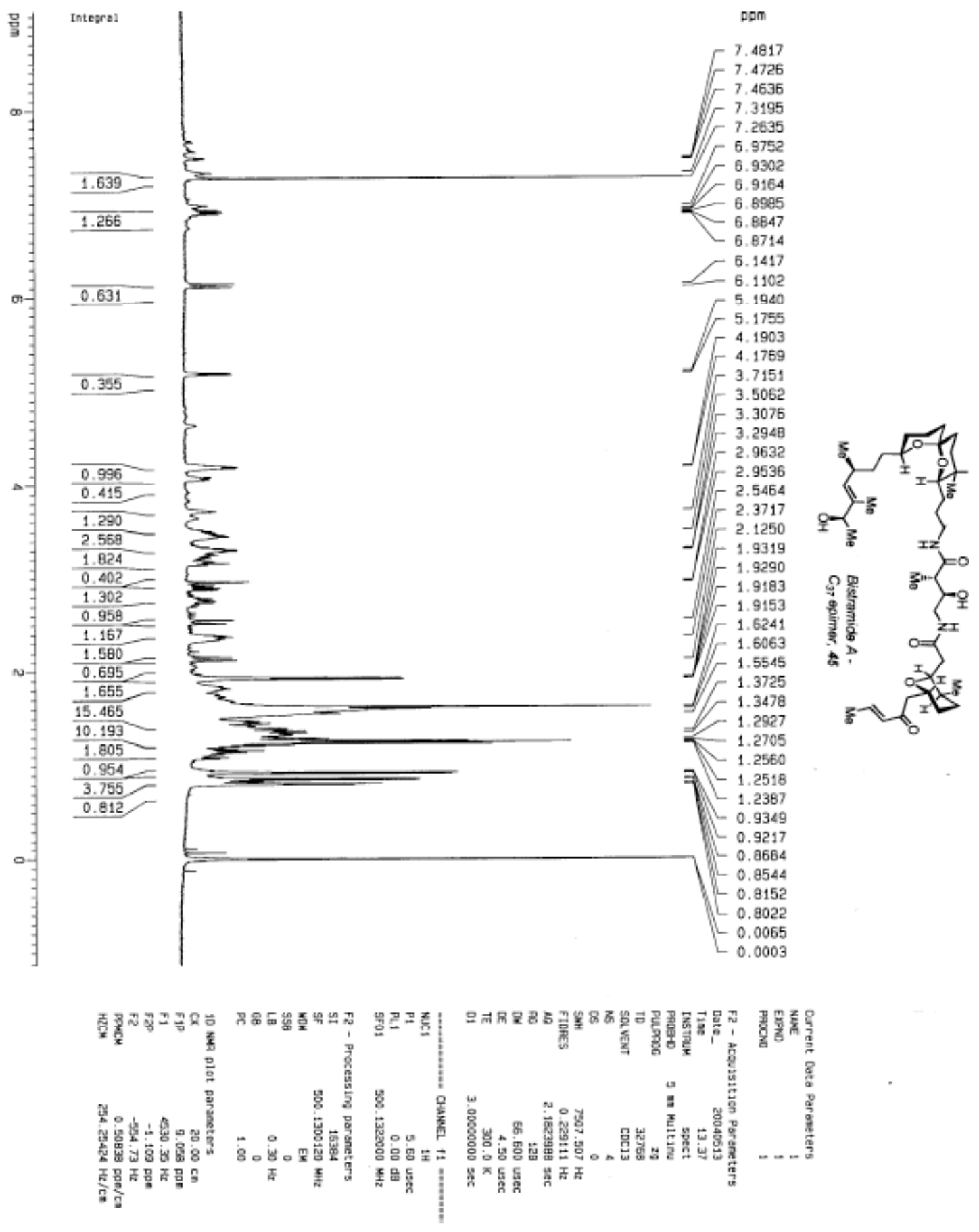




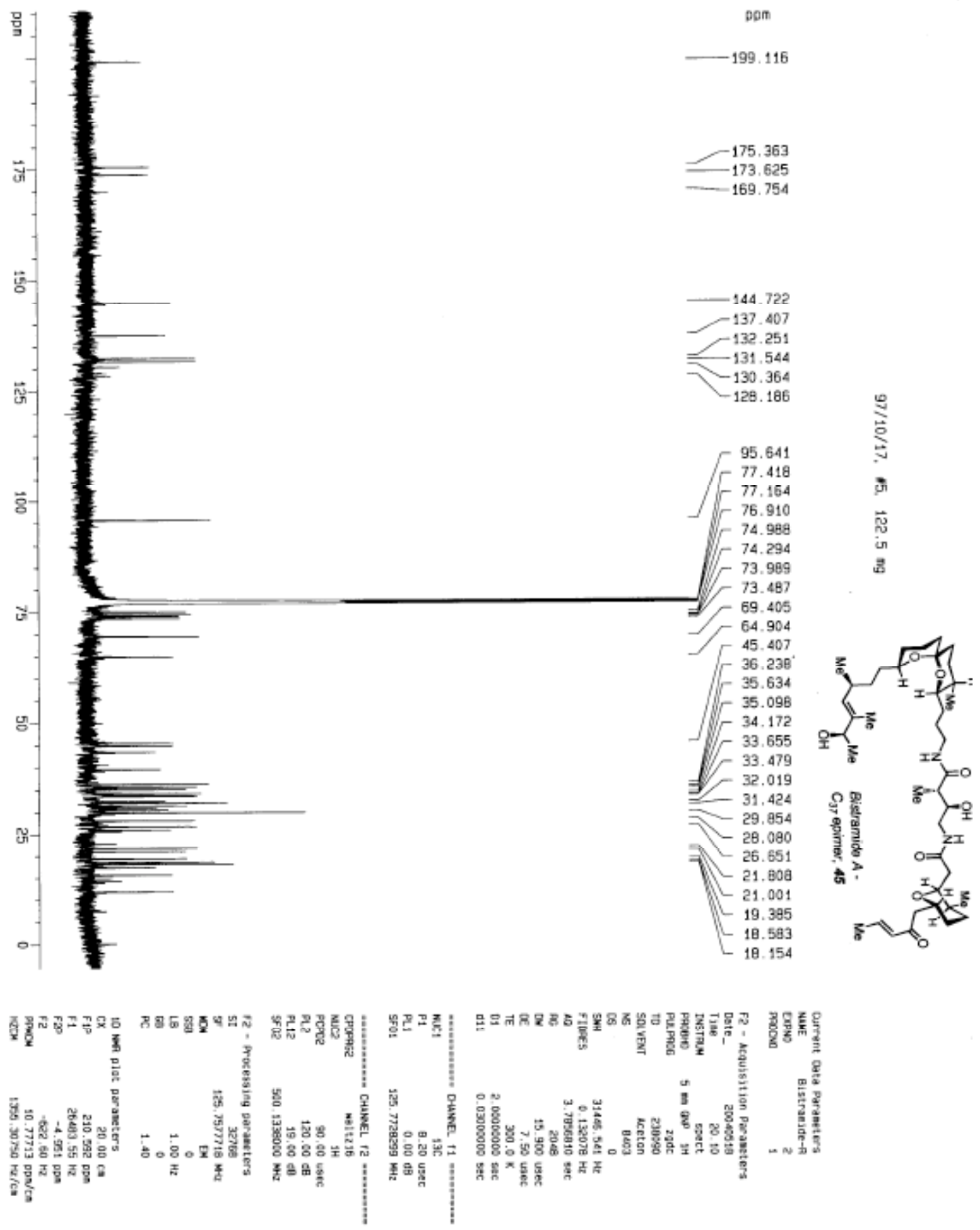

